\title{
Electromagnetic suspension and levitation
}

\author{
B V Jayawant \\ School of Engineering and Applied Sciences, University of Sussex, Brighton BN1 9QT, UK
}

\begin{abstract}
The phenomenon of levitation has attracted attention from philosophers and scientists in the past. The recent advances, notably in power electronics and magnetic materials, have focused this attention within the last decade on the application of electromagnetic suspension and levitation techniques to advanced ground transportation. Regardless of the fact that there is, in effect, a separate technology involved for each electromagnetic method, the whole subject is given a blanket title of 'maglev'. There is also a very wide range of industrial applications to which magnetic suspension techniques could be profitably applied, particularly in the area of high-speed bearings to reduce noise and to eliminate friction, and yet only high-speed ground transportation has caught the imagination of the media. This review deals with the physics and engineering aspects of the four principal contenders for advanced ground transportation systems and describes the most up-to-date developments in Germany, Japan, USA and the UK in this field. This article also describes some of the very recent challenging developments in the application of electromagnetic suspension and levitation techniques to contactless bearings. A fairly comprehensive bibliography is given to enable the more interested reader to pursue the topic further in any one of the technologies dealt with in this review.
\end{abstract}

This review was received in April 1980. 


\section{Contents}

1. Survey of electromagnetic methods

2. Principles and limitations of electromagnetic techniques of suspension and levitation

2.1. Suspension or levitation using permanent magnets

2.2. Levitation using diamagnetic materials

2.3. Levitation using superconductors

2.4. Levitation using induced eddy currents

2.5. Levitation using forces acting on current-carrying conductors situated in magnetic fields

2.6. Suspension using a tuned $L, C, R$ circuit and an electrostatic force of attraction

2.7. Suspension using a tuned $L, C, R$ circuit and an electromagnetic force of attraction

2.8. Suspension using controlled DC electromagnets

2.9. Combined suspension and propulsion schemes

2.10. The mixed $\mu$ system of levitation

2.11. Contending systems for practical applications including advanced ground transportation

425

3. Levitation using permanent magnets

3.1. Properties of permanent magnets and magnetic materials 426

3.2. Permanent magnets for repulsion levitation 428

4. Levitation using superconducting magnets $\quad 432$

4.1. Some properties of superconductors $\quad 432$

4.2. Principles of superconducting levitation 435

5. Levitation using eddy currents induced by mains frequency excitation 447

5.1. Some stable and unstable AC induction levitators 447

5.2. Levitation of passenger carrying vehicles, or the magnetic river 453

$\begin{array}{ll}\text { 5.3. The magnetic river as a vehicle system } & 457\end{array}$

6. Suspension using controlled DC electromagnets 458

6.1. Principle of suspension using controlled DC electromagnets 458

6.2. Analytical aspects of multimagnet systems 462

6.3. Transducers, magnets and power amplifiers for magnetic suspension. systems

6.4. Contactless support and frictionless bearing applications of controlled DC electromagnetic suspension

7. Assessment of electromagnetic suspension and levitation schemes 472 


\section{Survey of electromagnetic methods}

The phenomenon of levitation has fascinated philosophers through the ages and it has attracted much attention from scientists in recent times as a means of eliminating friction or physical contact. Whilst the area of frictionless bearings is at least as important, it is the application of suspension and levitation to high-speed ground transportation which has received most attention, especially in the popular media. Regardless of the method employed the vehicles are described as 'hover trains' and any electromagnetic method is ascribed the title 'maglev'. To indicate the dislike the author has for this term this is the last time that the term will be used in this review. Technically, each method of suspension and levitation is a technology in its own right and it is, in the author's opinion, quite wrong to ascribe an all-enveloping title in any case.

Besides the air-cushion principle of supporting rotating shafts or vehicles as in the Aerotrain in France or the Tracked Hovercraft in this country there are nine other electromagnetic methods of supporting moving or rotating masses (Geary 1964, Jayawant 1981):

(i) Repulsion between magnets of fixed strength and of ferromagnetic materials.

(ii) Levitation using forces of repulsion and diamagnetic materials.

(iii) Levitation using superconducting magnets.

(iv) Levitation by repulsion forces due to eddy currents induced in a conducting surface or a body.

(v) Levitation using force acting on a current-carrying linear conductor in a magnetic field.

(vi) Suspension using a tuned $L, C, R$ circuit and the electrostatic force of attraction (between two plates).

(vii) Suspension using a tuned $L, C, R$ circuit and the magnetic force of attraction (between an electromagnet and a ferromagnetic body).

(viii) Suspension using controlled DC electromagnets and the force of attraction between magnetised bodies.

(ix) Mixed $\mu$ system of levitation.

Some of the possible methods of suspension or levitation in the above list are really of only academic interest but three in particular have been pursued with great vigour within the last decade with the application to advanced ground transportation schemes as the principal objective. It is necessary to distinguish at the outset between those methods which use forces of attraction and those which use forces of repulsion. The former may be called suspension techniques and the latter levitation.

\section{Principles and limitations of electromagnetic techniques of suspension and levitation}

It appears that every one of the methods listed above has been the subject of some enthusiastic investigation at one time or another. The difficulties of achieving stable suspension or levitation are, however, highlighted by an examination of the nature of forces when an inverse square law relates force and distance. Earnshaw's (1842) paper on the subject is now considered a classic by all workers in the field of electromagnetic 
suspension. This paper shows mathematically that it is impossible for a pole placed in a static field of force to have a position of stable equilibrium when an inverse square law operates and this fundamental calculation is known as 'Earnshaw's theorem'.

It is known in applied mechanics that a body is in equilibrium when the resultant of forces acting on it is zero. Furthermore, the state of equilibrium is stable, unstable or neutral depending on whether the body, if slightly displaced, would tend to return to the position of equilibrium, would tend to move further away from it or would not tend to move at all (Temple and Bickley 1933). In order to express this in terms of field theory we consider a particle, i.e. a body of negligible dimensions, placed at a point $\left(x_{0}, y_{0}, z_{0}\right)$ in a static field of force $F(x, y, z)$. The force on the particle is thus $F\left(x_{0}, y_{0}, x_{0}\right)$. If $\left(x_{0}, y_{0}, z_{0}\right)$ is to be a position of stable equilibrium two following conditions must be satisfied:

$$
F\left(x_{0}, y_{0}, z_{0}\right)=0 \quad \nabla \cdot F\left(x_{0}, y_{0}, z_{0}\right)<0 .
$$

The first is a condition of equilibrium and the second, a condition of stability. Moreover, if $F$ is an irrotational field then

$$
F(x, y, z)=-\nabla \psi(x, y, z)
$$

where $\psi$ is a potential. In terms of $\psi$ the necessary conditions for stable equilibrium are

$$
\nabla \psi\left(x_{0}, y_{0}, z_{0}\right)=0 \quad \nabla^{2} \psi\left(x_{0}, y_{0}, z_{0}\right)>0 .
$$

Earnshaw's theorem is essentially an extension to electromagnetic fields of those conditions which can be rigorously proved using potential theory (Kellogg 1953, Papas 1977). In a charge-free region $R$ the electrostatic field $E(x, y, z)$ is solenoidal and irrotational, i.e.

$$
\nabla \cdot E(x, y, z)=0 \quad \nabla \times E(x, y, z)=0 .
$$

From the second of these equations it follows that

$$
E(x, y, z)=-\nabla \varphi(x, y, z)
$$

where $\varphi$ is the electrostatic potential. The force on a particle of charge $q$ placed in the field is

$$
F(x, y, z)=q E(x, y, z) .
$$

Taking the divergence of this equation and considering the first of equations (2.4)

$$
\nabla \cdot F(x, y, z)=0
$$

for all points in $R$. Thus, although equation (2.6) may satisfy the first of the two conditions (2.1) necessary for stable equilibrium, (2.7) violates the second. Thus a charged body placed in an electrostatic field cannot rest in stable equilibrium under the influence of electric forces alone. The theorem is of wider applicability than electrostatic fields, for example to the Newtonian potential of gravitational theory.

Braunbeck (1939a, b) extended the analysis to uncharged dielectric bodies in electrostatic fields and magnetic bodies in magnetostatic fields. The distinguishing feature of these cases is that they involve dipoles whereas Earnshaw's theorem applies to individual particles.

When a dielectric body is placed in an electrostatic field the polarisation $P$ is related to the electric field $E$ by

$$
P=\chi_{\mathrm{e}} E
$$


where $\chi_{\mathrm{e}}$ is the electric susceptibility of the dielectric body. The induced dipole moment $p$ of the body is given by

$$
p=\int P \mathrm{~d} V
$$

over $V$, the volume of the body. Assuming that the body is small enough for $E$ to remain constant

$$
p=\int \chi_{\mathrm{e}} E \mathrm{~d} V=\chi_{\mathrm{e}} E V \text {. }
$$

The force on the body is given by

$$
F_{\mathrm{e}}=(p \cdot \nabla) E
$$

which, with the aid of equation (2.10), yields

$$
F_{\mathrm{e}}=\chi_{\mathrm{e}} V(E \cdot \nabla) E .
$$

Since $\chi_{\mathrm{e}}=\left(\varepsilon_{r}-\varepsilon_{0}\right)$ where $\varepsilon_{r}$ is the dielectric constant of the body and $\varepsilon_{0}$ is the dielectric constant of free space and since $(E \cdot \nabla) E=\frac{1}{2} \nabla E^{2}$ equation $(2.12)$ can be rewritten as

$$
F_{\mathrm{e}}=\frac{1}{2}\left(\varepsilon_{r}-\varepsilon_{0}\right) V \nabla E^{2} \text {. }
$$

Equation (2.13) gives the force that a dielectric body of volume $V$ and dielectric constant $\varepsilon_{r}$ experiences in an electrostatic field $E$. Similarly a magnetic body in a magnetic field $H$ experiences a force

$$
F_{\mathrm{m}}=\frac{1}{2}\left(\mu_{r}-\mu_{0}\right) V \nabla H^{2}
$$

where $\mu_{r}$ is the permeability of the body and $\mu_{0}$ is the permeability of free space.

Since the divergence of $\nabla E^{2}$ can nowhere be negative and since it is physically impossible for $\left(\varepsilon-\varepsilon_{0}\right)$ to be negative, it means that the condition given by equation $(2.4)$ cannot be satisfied and hence a dielectric body cannot be in stable equilibrium anywhere in the electrostatic field.

For magnetic bodies the situation is quite different. Although the divergence of $\nabla H^{2}$ can nowhere be negative the quantity $\left(\mu_{r}-\mu_{0}\right)$ can be negative for diamagnetic and superconducting bodies as well as effectively so for conducting bodies with induced eddy currents. Thus condition (2.4) can now be satisfied and stable suspension is possible for diamagnetic bodies and for superconducting bodies, as well as for conducting materials such as, say, aluminium or copper in the proximity of coil systems carrying alternating currents.

\subsection{Suspension or levitation using permanent magnets}

It follows from Earnshaw's theorem and Braunbeck's analysis that stable suspension or levitation is impossible with a system of permanent magnets (or fixed-current electromagnets) unless part of the system contains either diamagnetic material $\left(\mu_{r}<1\right)$ or a superconductor $\left(\mu_{r}=0\right)$ and that it is altogether impossible to achieve suspension or levitation in electrostatic fields since there are no known materials with $\varepsilon_{r}<1$.

Early work on the use of permanent magnets from about 1890 was concerned with taking part or whole of the load of a rotor shaft magnetically. In spite of the large number of patents none of this work appears to have been commercially successful. Interest in this topic seems to have languished until the 1930s and the advent of improved permanent magnet materials. The commonest application of magnets of fixed strength put forward has been for the suspension of shafts or spindles of watt hour meters to 
relieve the load on the pivot bearing, a constant target of magnetic suspension workers (Evershed 1900, Faus 1943, de Ferranti 1947). Further possible applications in the aerospace field are discussed in a General Electric Co. (USA) report (1963).

Recent developments in permanent magnets fabricated from high coercivity ferrite materials has once again raised the subject of using them for levitation of vehicles to carry passengers. Polgreen $(1965,1966 \mathrm{a}, \mathrm{b})$ was the first to propose application to a trackbound vehicle using newly developed $\mathrm{BaF}_{3}$ magnets (Polgreen 1968, 1971) in the repulsion mode. His was a model system consisting of blocks of barium ferrite magnets fixed to the underside of a vehicle with nylon rollers for guidance. Similar proposals, including one for high-speed travel across the USA in evacuated tubes, were made by others at about the same time (Westinghouse Engineer 1965, Baran 1971, Forgacs 1973). In all these proposals it has been assumed that barium ferrite would be cheap to manufacture in large quantities required for laying down the track. One of the advantages of using ferrites for track is that there are no induced eddy currents. Thus there is no drag force or a loss of lift due to eddy current reaction.

McCaig (1961) and Bahmanyar and Ellison (1974) have made a study of the lifting forces, configurations and track designs for tracks constituted of permanent magnets. Any practical systems built around these ideas, however, would require damping in the vertical direction and guidance as well as damping in the lateral direction. This has been considered only by Voigt (1974) who proposed driving a current proportional to vertical velocity in a coil around the permanent magnets. Recently a new magnetic material, samarium cobalt, with an even greater coercivity than barium ferrite has appeared. The intrinsic coercive force of these cobalt rare-earth materials may be 20-50 times that of conventional permanent magnets and lifting capabilities in repulsion mode of 5-10 times (Becker 1970). In spite of these significant advances in materials there remain many practical difficulties in the implementation of transportation schemes using permanent magnet tracks. However, for instrument-bearing applications there exists a real possibility of using controlled permanent magnet schemes whereby the power consumption in the steady state can be made virtually zero.

\subsection{Levitation using diamagnetic materials}

Levitation can be achieved as indicated earlier in static magnetic fields by employing diamagnetic materials but even the two materials which exhibit most pronounced diamagnetic properties, bismuth and graphite, are so weakly diamagnetic that only small pieces of diamagnetic materials can be levitated.

The topic of levitation in the presence of diamagnetic materials has been studied by Braunbeck (1939a, b, 1953) and Boerdijk (1956a, b). Braunbeck levitated small pieces of bismuth $0.75 \mathrm{~mm} \times 2 \mathrm{~mm}$, weighing $8 \mathrm{mg}$, and graphite $2 \mathrm{~mm} \times 12 \mathrm{~mm}$, weighing $75 \mathrm{mg}$, between specially formed poles of an electromagnet capable of producing a field of flux density $2.3 \mathrm{~T}$. Boerdijk repeated this experiment on a smaller scale using permanent magnets and also performed an alternative experiment of levitating a magnetised disc of $1 \mathrm{~mm}$ diameter between a magnet attracting it upwards and a piece of diamagnetic material below it. His analysis concluded that it should be possible to levitate a magnetised particle of micrometric size a fraction of a millimetre above a piece of bismuth or graphite without the aid of a surmounted magnet. A further examination of diamagnetic levitation is contained in a report of the General Electric Co. (USA) but the broad conclusions to be drawn from the results of these workers is inevitably that the phenomenon of diamagnetic levitation is of no more than academic interest. 


\subsection{Levitation using superconductors}

Certain metals and alloys when cooled to a temperature approaching $0 \mathrm{~K}\left(-273^{\circ} \mathrm{C}\right)$ become superconductors. The superconducting state is indicated by the complete absence of electrical resistance and once initiated a current will continue to flow without the presence of a voltage source in the circuit. This is also accompanied by rejection of magnetic flux in the superconducting body and is known as the Meissner effect (Meissner and Ochsenfeld 1933) which causes superconductors to behave as perfectly diamagnetic materials $\left(\mu_{r}=0\right)$. Stable suspensions using permanent magnets are, therefore, possible.

The first recorded demonstration of this principle was the levitation of a $15 \mathrm{~mm}$ bar over a superconducting lead plate by Arkadiev (1945). In the subsequent pursuit of the development of a cryogenic magnetically levitated gyroscope superconducting spheres were levitated over various arrangements of electromagnets (Simon 1953, Culver and Davis 1957). A scheme for levitating a vehicle over two parallel superconducting rails
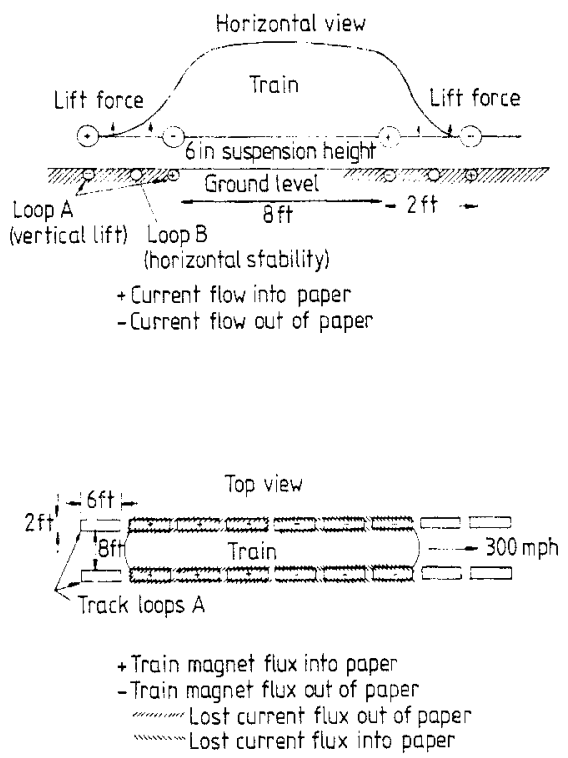

Figure 1. Conceptual view of track and train as proposed by Powell and Danby (1966).

was proposed by Powell in 1963 and later (Powell and Danby 1966) a second system (figure 1) in which there was no need for superconducting rails, as attached to the vehicles would be superconducting magnets, which would ride over normal conducting rails without touching them. There were proposals (Guderjahn et al 1969) to support a rocket launching sledge (figure 2) capable of speeds of $5 \mathrm{~km} \mathrm{~s}^{-1}$ and further studies of baseline specifications for passenger carrying vehicles (Borcherts et al 1973).

The electrodynamically levitated vehicle, as it is known, is lifted and guided by repulsion forces between superconducting magnets on the vehicle and secondary circuits on the track, or eddy currents if the track is passive. The levitation is self-stabilising and clearance between magnets and secondary circuits can be larger than $10 \mathrm{~cm}$. However, the stiffness and damping of the suspension are low and also the vehicle must be in motion to generate lift. There is, therefore, a minimum velocity which must be exceeded before the vehicle becomes levitated and the system is generally considered suited to high-speed transport schemes travelling at speeds in excess of $300 \mathrm{~km} \mathrm{~h}^{-1}$. Many problems remain 


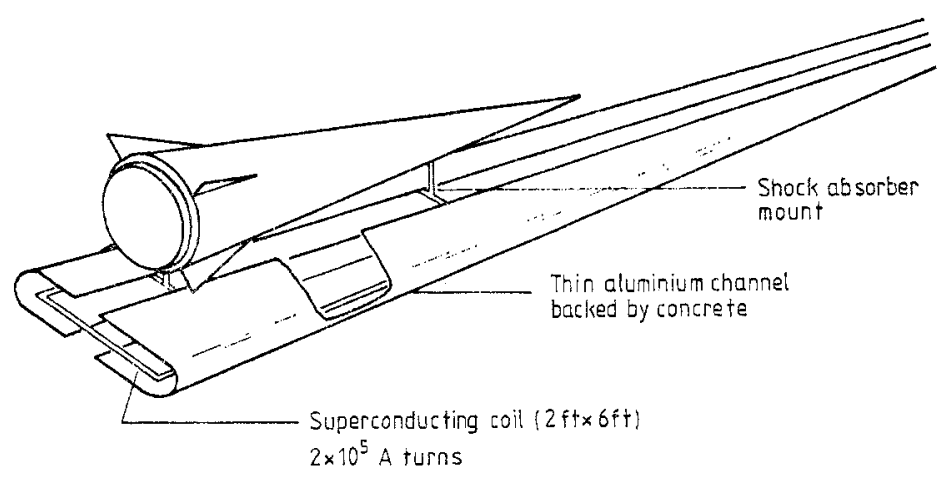

Figure 2. Superconducting rocket sledge.

unresolved as yet and among the principal ones is that of eddy current drag in addition to the aerodynamic drag on such vehicles. The eddy current drag is rather large at low speeds and this places quite a substantial burden on the propulsion systems during acceleration. The drag reduces at high speeds but in order to get a high lift to drag ratio (a figure of merit for these systems) a large quantity of conducting material (aluminium) is required in the secondary circuits (track). At high speeds the low inherent damping coefficient of the suspension or guidance further reduces and in fact can become negative, presenting some quite serious problems of vehicle stability in general. It has been reported that passive damping may be inadequate (Borcherts et al 1973, Thornton 1973, Ellison and Bahmanyar 1974, Ohno et al 1973, Coffey et al 1969). If a linear synchronous motor is used as propulsion unit, a proposal to vary the drive to the motor in accordance with the vertical acceleration signals fed back (Greene 1974) or variation of coil currents (Ooi and Banakar 1975) to produce more damping at the expense of the figure of merit have been considered.

Research on superconductive levitation schemes is quite active in Canada, Japan and England. The Japanese National Railways produced a $3 \frac{1}{2}$ ton vehicle in 1972. It had a lift of $6 \mathrm{~cm}$ but guidance was provided by wheels on the sides of the guideway. A second and more advanced vehicle (Outsuka and Kyotani 1975, Yamamura and Ito 1975) operating on a $20 \mathrm{~km}$ track (figure 3 (plate)) has been reported in 1979 as having achieved speeds in excess of $500 \mathrm{~km} \mathrm{~h}^{-1}$.

There were two projects in the United States. One was a collaborative effort between various universities and industrial laboratories under the direction of the Department of Transportation. The other project, called the magnaplane project, was partly under the direction of the National Science Foundation. Both studies were theoretical as well as experimental but involving permanent magnets (Thornton 1973, Ooi and Banakar 1975, Tang et al 1975, Reitz and Borcherts 1975). Research in the United States appears to have been halted indefinitely since about 1975 .

Research in Canada on superconducting levitation systems for high-speed ground transportation with synchronous linear motor propulsion is being carried out by an interdisciplinary team of scientists and engineers from the universities of Toronto, Queen's and McGill (Eastham 1975). A $7.6 \mathrm{~m}$ diameter wheel rotating about a vertical axis with a maximum peripheral speed of $100 \mathrm{~km} \mathrm{~h}^{-1}$ is being used to carry out full-scale tests of propulsion, levitation and guidance systems (Slemon 1975).

In England work has been going on for a number of years (Eastham and Rhodes 1971, Rhodes et al 1974, Rhodes 1976) at the University of Warwick and a $600 \mathrm{~m}$ track 
has been constructed to test a small vehicle which initially is to be towed by a rope at speeds of up to $35 \mathrm{~m} \mathrm{~s}^{-1}$. This vehicle is $3 \mathrm{~m}$ long and weighs $150 \mathrm{~kg}$.

Studies are also being carried out by a consortium of Siemens, AEG and Brown Boveri in Germany at Erlangen and a vehicle has undergone preliminary tests on a 280 m diameter circular track (Guthberlet 1974, Uranker 1974). It is believed that, due to the unresolved problems of guidance and eddy current drag, the activity at Erlangen is now (1979) concentrated more on superconducting synchronous linear motors than on levitation. However, the Erlangen vehicle was reported as having achieved levitation at speeds in excess of $100 \mathrm{~km} \mathrm{~h}^{-1}$.

\subsection{Levitation using induced eddy currents}

A force of repulsion is generated between a coil carrying alternating current and an electrically conducting surface when placed in the proximity of the coil so that the alternating magnetic field of the coil induces eddy currents in the conductor. This effect can be utilised for the levitation of conducting objects and one of the early patents purporting to do so is that of Anschuitz-Kaemfe (1923a) in gyroscopic applications. This technique has also been used for simultaneous levitation and meiting of specimens (Orkress et al 1952)

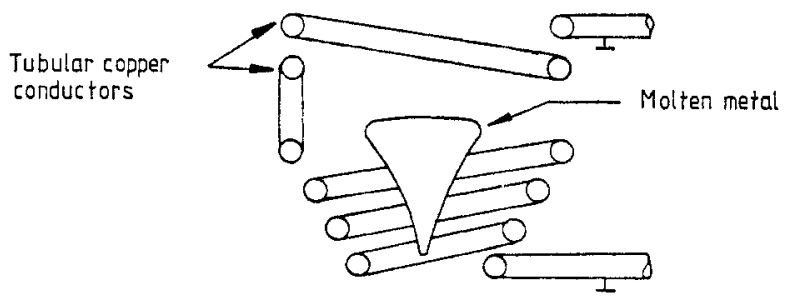

Figure 4. Levitation of moiten metal using eddy currents.

at $10 \mathrm{kHz}$ for zone refining of metals (figure 4). This technique is useful in laboratories for the preparation of small quantities of alloys without contamination from crucibles.

A plate levitator in which two concentric coils carry $50 \mathrm{~Hz}$ currents in opposite directions and can levitate a circular conducting plate in stable conditions is described by Bedford et al (1939) and several other experimental systems for levitation of plates, spheres, etc, are described by Laithwaite (1965). More recently, however, due to developments in linear induction motors, particularly of the transverse flux type (Laithwaite et al 1971, Eastham and Laithwaite 1973) it has been claimed that such machines might be used for combined levitation and propulsion of high-speed vehicles (Eastham and Laithwaite 1974). On the basis of a great deal of experimental work on relatively small models it is suggested that due to scaling laws for electromagnetic machines (Laithwaite 1973b) combined levitation and propulsion schemes, employing linear induction motors for vehicles weighing in excess of 50 tons, may have performances comparable to that of the superconducting magnet schemes. One of the advantages claimed for such schemes termed the 'magnetic rivers' is that they offer the possibility of lift and guidance where the motor necessary for propulsion is the source of such facilities. It is also claimed that for a particular thrust the secondary power input in a levitating linear motor will be the same as in a machine designed for thrust only. Obviously a great deal of work, particularly theoretical, needs to be done. It is not easy, none the less, to envisage such dramatic improvements to primary reactive power input for large airgap operation, claimed as one 
of its advantages, as to make the performance extrapolated from small models seem unrealistic. Results of a calculation by Eastham (1978) are given in $\$ 5.3$ and they largely bear out the pessimism expressed here. The ideas involved are, however, extremely ingenious and regardless of the levitation aspects the use of transverse flux machines only as propulsion units remains very promising.

\subsection{Levitation using forces acting on current-carrying conductors situated in magnetic fields}

The force acting on a conductor of length $l$ carrying a current $I$ and situated in a transverse magnetic field of intensity $B$ is given by $B l I$ and the force acts in a direction normal to both the conductor and the magnetic field. Pfann and Hagelbarger (1956) report as having supported the molten portions of a metal rod undergoing zone melting by locating the molten portion in a transverse magnetic field and passing a current through the rod. Although the current is adjusted to give an upward force approximately equal to the weight of the molten metal surface tension also contributes to keeping the molten zone in place. The heating of the molten zone is carried out either by induction heating or by a torch flame. Thus, unlike the eddy current levitation technique the functions of melting and levitation are kept separate. Rods of iron, nickel and tin have been levitated by this method.

A variant of the same technique was proposed by Powell (1963) for the levitation of a vehicle over two parallel superconducting rails carrying a persistent current. Attached to the vehicle are two superconducting inverted troughs which ride over the rails without touching them. Levitation is effected by persistent currents flowing in the longitudinal wires of which the troughs are constructed. The troughs are designed to give the vehicle stable equilibrium both vertically and laterally. In his paper, which contains technical and economic calculations and a report of preliminary experiments, Powell estimates that, with a current of $300000 \mathrm{~A}$ and a trough radius of $18 \mathrm{in}$, a weight of $3400 \mathrm{lb} \mathrm{ft}^{-1}$ could be supported. The idea does not seem to have been taken up by anyone since its publication and a recent discouraging report about the prospects for superconducting cables (Skinner and Edwards 1978) would suggest that it is not likely to either, on both technical and economic grounds.

\subsection{Suspension using a tuned $L, C, R$ circuit and an electrostatic force of attraction}

An electrically conducting shaft or rotor may be held in suspension by electrostatic forces between a pair of electrodes where one of the electrodes is the body to be suspended. The suspended body and the fixed electrode form the capacitance element of a tuned $L, C, R$ circuit in such a manner that the potential difference between the two electrodes increases as the distance between them increases and vice versa, i.e. the circuit is tuned to resonate with capacitance values less than those at the suspension gap. The electrodes must be maintained at a potential difference of several kilovolts. The applications of this principle have been investigated for vacuum gyroscopes (Nordsiek 1961, Knobel 1964). This technique does not appear to have been pursued as extensively as the one using the magnetic force of attraction in tuned $L, C, R$ circuits. It is, however, almost certain that, besides the problem of high voltages required to achieve suspension, this method also suffers from inherent instability due to the use of tuned circuits and the problems of providing damping and high reactive power are just as adverse as in the $L, C, R$ systems employing variation of inductance with gap. 


\subsection{Suspension using a tuned $L, C, R$ circuit and an electromagnetic force of attraction}

As already indicated in the previous subsection this method has been investigated very extensively, particularly at MIT (Gillinson et al 1960, Frazier et al 1974) and the University of Virginia (1962), and also by Cambridge Thermionic Corp. (1963, 1975) and General Electric Co. (USA) (1963). Interest seems to have revived in this technique again in the late sixties in Japan (Hagihara 1974), Israel and the UK (Jayawant and Rea 1968, Kaplan 1967, 1970). The variation of inductance of an electromagnet in the proximity of a ferromagnetic body, depending on the separation between the two, is utilised in this method to regulate the current and hence the force of attraction. This is achieved (figure 5) by incorporating the electromagnet within an $L, C, R$ circuit tuned in such a way that when the object to be suspended moves away from the electromagnet the circuit tends to become resonant, thus increasing the current and hence the force acting on the object. Conversely, when the body moves towards the electromagnet the current and the

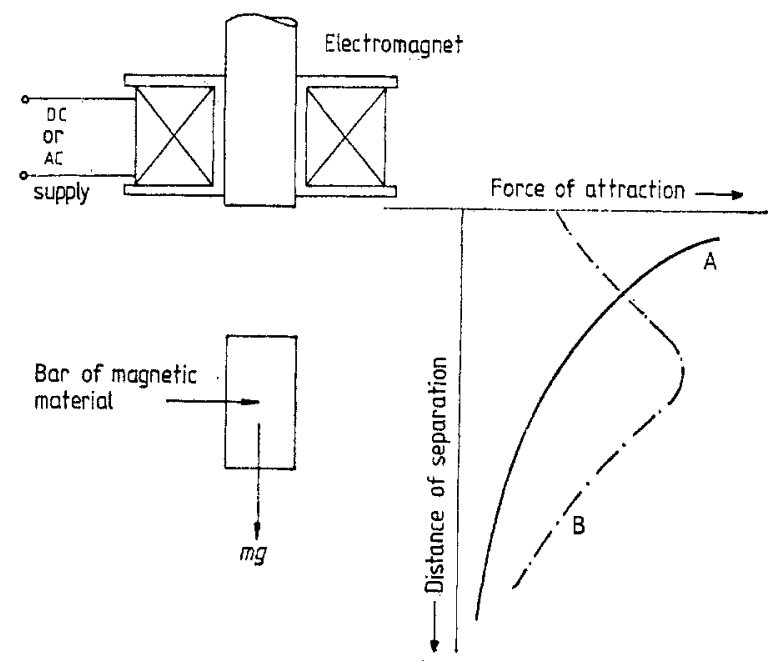

Figure 5. Geometry and force-distance curves. A DC excitation, B AC excitation with series capacitor.

force of attraction diminish. If, therefore, the force of attraction is balanced against that of gravity at some distance of separation it is possible to get a statistically stable sit point for the suspension of the body. However, tuned circuits possess large time constants which means that once disturbed from this static stable point the object usually goes into a divergent oscillation unless some means are employed to control and speed up the current changes or to provide damping in some other manner. Kaplan (1970) found that at frequencies of the order of $6-26 \mathrm{kHz}$ leaky capacitors ranging from $0.4-0.02 \mu \mathrm{F}$ provided adequate damping to obtain suspension of a ferrite disc and rod weighing $7.5 \mathrm{~g}$ and $13.5 \mathrm{~g}$, respectively. Others have used oil damping by submerging the body to be suspended in oil.

The stiffness of suspension using the $\mathrm{AC}$ tuned circuit method tends to be rather low for many applications. The main disadvantages, however, stem from the fact that at the static sit point the circuit is predominantly inductive and hence reactive power input is rather large and that the iron structure including the object to be suspended must be laminated. Thus, although this method seems to offer at first sight an inherently stable force-distance characteristic (Jayawant and Rea 1968) and, therefore, considerable 
advantages for the suspension of ferromagnetic bodies, rather disappointingly it suffers from severe drawbacks and thus has not resulted in any practical applications.

\subsection{Suspension using controlled DC electromagnets}

This method, at the present time, is by far the most advanced technologically and is the subject of world-wide investigation not only for advanced ground transportation schemes but also for application in contactless bearings for both high and very low speeds.

The first proposal for a controlled magnet attraction scheme appears to be by Graeminger (1912) for a vehicle suspended below an iron rail by a U-shaped electromagnet carried on the vehicle facing the underside of the rail. A gap was to be maintained between the electromagnet and the rail by a mechanical or fluid pressure-sensing device which would vary a resistance in series with the magnet winding or vary an airgap in the magnet core. As it stood the proposal did not have any practical potential. AnschuitzKaempfe (1923b) then suggested contactless centering of a floated sphere containing gyrorotors using electromagnets. Position sensing was to be achieved by measurement of the resistance of the conductive fluid between the inner and outer spheres. Alternating current was also to be supplied to the support rails so that the eddy currents induced in the inner sphere would centre it by repulsion. The first amongst the present generation of suspension schemes using active control of current in electromagnets, however, is probably due to Kemper $(1937,1938)$ who proposed a vehicle suspended by electromagnets attracting to the underside of a rail using either capacitive or inductive means of sensing distance below the rail. Part of the circuit also yielded a voltage proportional to the rate of change of the airgap for damping of the vertical oscillations. Kemper constructed a model consisting of an electromagnet with pole faces of $30 \mathrm{~cm} \times 15 \mathrm{~cm}$ and suspended a mass of $210 \mathrm{~kg}$. The airgap flux density was $0.25 \mathrm{~T}$, the airgap $15 \mathrm{~mm}$ and the power consumption $270 \mathrm{~W}$. This remained the heaviest weight to be suspended using any method of electromagnetic suspension or levitation until the demonstration of their 6.5 ton vehicle in 1971 by Messerschmitt Bölkow-Blohm (MBB) in West Germany.

Much of the published work after that of Kemper on the development of the electromagnetic suspension scheme using controlled DC electromagnets and external positionsensing was at the University of Virginia, particularly on rotor suspensions. The work carried out by Holmes (1937) and Beams (1937) was for rotors of high-speed centrifuges required in the fields of biology and medicine, typical speeds being 77000 RPM for a $3.97 \mathrm{~mm}$ diameter rotor. The other applications proposed were for testing bursting speeds of spheres such as ball bearings, testing adhesion of metal films, turbo-molecular pumps for use at high vacuum free of bearings requiring lubricants, and magnetic suspension balances capable of recording weight changes of $5 \times 10^{-11} \mathrm{~g}$ in a suspended weight of $2.3 \times 10^{-6} \mathrm{~g}$.

The same principle has been used to suspend aircraft models in wind tunnels (Tournier and Laurenceau 1957, ONERA 1960) and appears to be the first instance of control of the three degrees of freedom of a suspended body. Since the objectives are to determine the forces acting on the aerodynamic model the system is in effect a balance. Apart from the fact that it is virtually impossible to make an interference-free wake-flow field without a suspension system, the accuracy of such a scheme is more compatible with recent requirements in aerodynamics. Further magnetic suspension helps the investigation of more subtle aerodynamic details and improves techniques for studying aerovehicle stability (Clemens and Cortner 1963, Covert and Finston 1973). The importance of the method can be seen by the fact that all major aerodynamic research 
centres in the world have resorted to it at one time or another. Although this application appears to have originated in France (Tournier and Laurenceau 1957, ONERA 1960) it was soon taken up by others; in the UK at the University of Southampton (Judd and Goodyear 1965) and the RAE (Wilson and Luff 1966); in the US at MIT-ARL (Chrisinger et al 1963), AEDC (Crain 1965), University of Virginia (Jenkins and Parker 1969), Princeton University (Dukes and Zapata 1969), University of Michigan (Silver and Henderson 1969) and NASA (Kilgore and Hamlet 1966).

There has been considerable activity since 1971 in the field of advanced ground transportation schemes using controlled DC electromagnetic suspension, the first demonstration being that of the 6.5 ton vehicle by MBB operating on a $700 \mathrm{~m}$ track. This was closely followed by another demonstration in Germany by Krauss Maffei in 1972, by the author (Jayawant et al 1975) at the University of Sussex (figure 6 (plate)), Japan Air Lines and General Motors in 1975 and, finally, British Rail (Linder 1976). It was reported in 1977 that the two separate developments in Germany had been merged into one programme and that this consortium had tested (Gottzein and Crämer 1977) a rocketpropelled vehicle Komet II on a $20 \mathrm{~km}$ track at speeds in excess of $400 \mathrm{~km} \mathrm{~h}^{-1}$. They also demonstrated a 68 passenger, 35 ton vehicle on a $700 \mathrm{~m}$ track at a transport exhibition in the summer of 1979 (figure 7 (plate)). Now AEG, Siemens and Brown Boveri, besides MBB and Krauss Maffei, are involved in the development of a $31.5 \mathrm{~km}$ track between Meppen and Papenberg in Emsland and a 121 ton vehicle is under construction (figure 8 (plate)). This is due for tests in 1982 and the unusual feature of this scheme is that the track is to have an air-cored winding of a (long stator) linear motor whereas the vehicle will have superconducting excitation magnets, i.e. the drive will be a long stator linear synchronous motor.

There were two development projects in Japan; one appears to be a joint universityindustry collaborative venture which produced a 1.8 ton vehicle, whilst in the more widely known development of Japan Air Lines the 1 ton vehicle has been followed by the demonstration of a 2.3 ton, $7 \mathrm{~m}$ long coach (figure 9 (plate)) capable of carrying eight passengers (Nakamura 1979). This development is specifically aimed at linking the two airports of Tokyo, one at Nerita and the other at Haneda, and plans are that these links will be operational by 1985 .

\subsection{Combined suspension and propulsion schemes}

Although not very far advanced some interesting proposals have recently arisen (Ross 1973, Eastham 1977, Edwards and Antably 1978) for combining the two functions, propulsion and suspension, into one. The first to investigate this were Rohr Industries who demonstrated a 3.6 ton vehicle which uses a linear induction motor for both propulsion and lift. The disadvantage of this proposal is that the track has to have 'rotor' bars to enhance the induction action. On top of this, linear induction motors are not necessarily the most ideal form of propulsion unit for high-speed vehicles. Alternatives have been suggested by Edwards and Antably (1978) and Eastham (1977) to use either reluctance machines or synchronous machines. The linear reluctance machine would require inert steel segments embedded in the track and control of both voltage and frequency applied to the 'stator' on the vehicle; voltage to control the suspension gap and frequency to control the speed. With the linear synchronous motor it is possible to put both DC (excitation) and AC windings on the same member (figure 10) and then the iron plates may be embedded in the track whereas the wound members are on the vehicle. It is also claimed that there is very little weight penalty arising out of the additional DC windings 
on the same frame. Unlike the Rohr Industries investigation the Edwards and Eastham proposals are very much at an early experimental stage but look very promising none the less.

The vehicle demonstrated by MBB (figure 7) in Hamburg in June 1979 was also propelled by linear synchronous motors. The two stator windings are on the track and thus the motors may be called long stator motors. The DC lift magnets are small modules and act as excitation magnets for the synchronous motors whilst attracting against the face of the laminated long stator. This construction would, on the face of it, appear to be very expensive in track construction. Hence the proposal to employ air-cored windings for the linear motor(s) on the track and superconducting excitation magnets on the vehicle in Emsland would seem to be more economical as well as eliminating the need

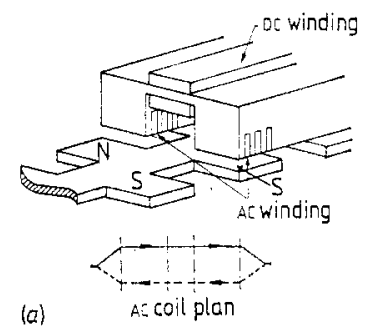

(a)

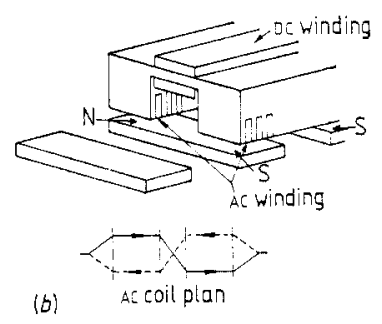

Figure 10. Possible geometrics of linear synchronous motors with passive track. (a) Homopolar-inductortype linear synchronous motor, $(b)$ transverse-flux homopolar machine.

to pick up power for the propulsion units through sliding contacts at high speeds, a problem which as yet has not really been solved.

\subsection{The mixed $\mu$ system of levitation}

Earnshaw's discussion (1842) of the stability of bodies in inverse square law fields showed that any equilibrium in such fields is not stable. This result also applies to fixed charge or current distributions in free space but not to systems whose permeability differs from that of free space. Braunbeck's (1939a, b, 1953) extension of this theoretical analysis is that where permittivity or permeability of the system is somewhere less than that of free space, when dielectric or magnetic bodies are present, stability is possible. Bevir (1976) has recently examined in more detail mixed systems where permeability in some places is less than that of free space but is greater in some other places and has shown that in a few cases stable suspension can be obtained.

A necessary but not sufficient condition for stability is $\nabla \boldsymbol{F}<0$ where $\boldsymbol{F}$ is the force 
vector acting on a body and the following table (Rutherford Laboratory and Culham Laboratory 1976) indicates how the sign of $\nabla F$ may depend only on the nature of the system and not on its geometry. This table, therefore, indicates that there exists a class

Table 1.

\begin{tabular}{llll}
\hline System & Permeability & $\nabla F$ & Stability \\
\hline $\begin{array}{l}\text { Normal conductors with } \\
\text { constant current and } \\
\text { orientation }\end{array}$ & $\mu=\mu_{0}$ & 0 & Marginally stable \\
$\begin{array}{l}\text { Constant current coils } \\
\text { and iron }\end{array}$ & $\mu>\mu_{0}$ & $>0$ & Unstable \\
$\begin{array}{l}\text { Constant current coils with } \\
\text { superconductors, eddy } \\
\text { currents or constant flux coils } \\
\begin{array}{l}\text { Mixed system of coils, iron } \\
\text { and superconductors }\end{array}\end{array} \quad \begin{array}{l}\mu>\mu_{0} \\
\text { and } \\
\mu<\mu_{0}\end{array}$ & $\begin{array}{l}>0 \\
\text { and } \\
<0\end{array}$ & $\begin{array}{l}\text { Stable operation } \\
\text { possible }\end{array}$ \\
& in different regions & $\begin{array}{l}\text { Stable operation } \\
\text { possible }\end{array}$ \\
\hline
\end{tabular}

of mixed systems with materials both of $\mu>\mu_{0}$ and $\mu<\mu_{0}$, i.e. iron and superconductors as well as normal conductors with eddy currents or constant flux coils which should provide stable suspensions.

These theoretical predictions have been verified (Homer et al 1977) in three small-scale experiments (figure 11 (plate)). In the first experiment iron washers or nuts weighing $1.5 \mathrm{~g}$, in the second experiment small iron disc $27 \mathrm{~mm}$ diameter $\times 20 \mathrm{~mm}$ long but using both a superconducting coil and a superconducting screen, whilst in a third experiment using two superconducting coils a much bigger iron cylinder $110 \mathrm{~mm}$ diameter $\times 40 \mathrm{~mm}$ long weighing $100 \mathrm{~g}$ have been stably suspended. The geometry of the third experiment is claimed as the more suited to passenger vehicle application. No results are as yet available for this configuration nor is it easy to visualise a geometry which would be suitable for the vehicle application. In any case, a great deal of work is needed not only on larger models but also on the control aspects before claims for the superiority of this method over others can be taken seriously. It is, however, an extremely ingenious technique.

\subsection{Contending systems for practical applications including advanced ground transporta- tion}

Whilst all the systems described in $\$ 1.1$ have been thought of at one time or another as capable of practical application only three or four remain in contention as applicable to advanced ground transportation or vehicles capable of carrying passengers. Besides the vehicle application there remains the important area of contactless bearings. This has not received nearly as much attention from the popular media, which is to be expected, but also from industry where the possibilities of contactless suspension and the benefits accruing therefrom are limitless.

Attention will be focused mainly on the methods below in the following sections although some of the other methods will be expounded more fully in an appropriate 
context. The four methods are (i) use of permanent magnets in repulsion, (ii) use of superconducting magnets, (iii) use of mains frequency currents to generate eddy currents and repulsion forces, and (iv) use of controlled DC electromagnets.

\section{Levitation using permanent magnets}

Within the last decade or so a new class of materials for making permanent magnets has been developed based on cobalt and some rare-earth elements. The improvement is so great that the cobalt-rare-earth magnets are in a class by themselves. In terms of their resistance to demagnetisation the new materials are 20-50 times superior to the previous best Alnico and their magnetic energy is 2-6 times greater. Whilst, therefore, there have been innumerable attempts to use the force of repulsion between permanent magnets for applications such as load relief in bearings (Geary 1964, Jayawant 1981), the older materials have suffered from drawbacks of demagnetisation, if not actual reversal of one of the magnets in the case of a mismatch, and relatively weak forces of repulsion. The new cobalt-rare-earth materials have now radically altered this picture. Like poles of such magnets can be made to approach without suffering more than a small percentage loss in magnetism and even this loss is mainly reversible. Such magnets can be brought together an infinite number of times and after the first two or three times the force of repulsion does not change. A current-carrying coil on one of these magnets can be energised in a direction completely reversing the effective polarity of the combination and yet when the current in the coil is removed the magnet remains virtually unaffected.

The design of magnets for repulsion devices or for the applications involving combination with current-carrying coils involves new ideas and new methods as well as some understanding of the magnetic properties of the new materials.

\subsection{Properties of permanent magnets and magnetic materials}

Ferromagnetic elements have atoms in which one electron shell contains fewer than the maximum number of electrons. In such unfilled shells there are one or more unbalanced electron spins giving rise to a small magnetic moment and making the atom itself a tiny magnet. Ordinarily in a large collection of such atoms the atomic magnets point in various directions and cancel one another. If a sample of ferromagnetic material is placed in a magnetic field, however, the individual atomic magnets tend to line up so that when the sample is removed from the field, it retains a net residual magnetism. The total magnetisation indicated by the symbol $M$ is the sum of the contributions of all the elementary atomic magnets. Magnetisation in any direction reaches its saturation value when all the atomic magnets are parallel and pointing in that direction.

Magnetic materials are divided into two categories, hard or soft, depending on the ease with which they may be magnetised and demagnetised. A hard material has a wide hysteresis loop and a soft material has a narrow one. Each reversal of a loop represents energy lost. Therefore, soft materials are suited for certain electrical devices such as transformers where the material is subjected to a reversal of magnetisation many times a second. Hard materials, on the other hand, are what one is looking for in the applications of permanent magnets, particularly those utilising the forces of repulsion such as in suspension or levitation since the width of the hysteresis loop determines the intrinsic coercive force. This width can range from less than one hundredth of an oersted $\left(1 \mathrm{Oe}=79.6 \mathrm{~A} \mathrm{~m}^{-1}\right)$ in alloys used in telephone equipment to tens of thousands 
of oersteds in the new cobalt-rare-earth magnets. For comparison the Earth's magnetic field is just under half an oersted.

Magnetic materials are generally described by their hysteresis loops in which the vertical axis is the total flux density $B$ rather than the magnetisation $M$. The total fiux density includes the contributions of both magnetisation and field strength $H$. In electrical engineering the performance of devices is much more closely related to the total flux density. In the field of the physics of magnetic materials, however, the magnetisation $M$ is an indication of what the material does in response to a magnetic field and thus both $B$ and $M$ are useful.

The upper left-hand quadrant of a $B-H$ loop is important from the engineers' point of view in trying to determine the quality of permanent magnets. This is illustrated in figure 12. Three points on this part of the curve are significant. The first is the crossing of the vertical axis, i.e. where the magnetising field has been removed but the material retains its magnetisation. This is termed remanence $B_{\mathrm{r}}$. If, now, the magnetising field is actually reversed, the value of the imposed field, $H_{\mathrm{c}}$, which reduces the total flux density

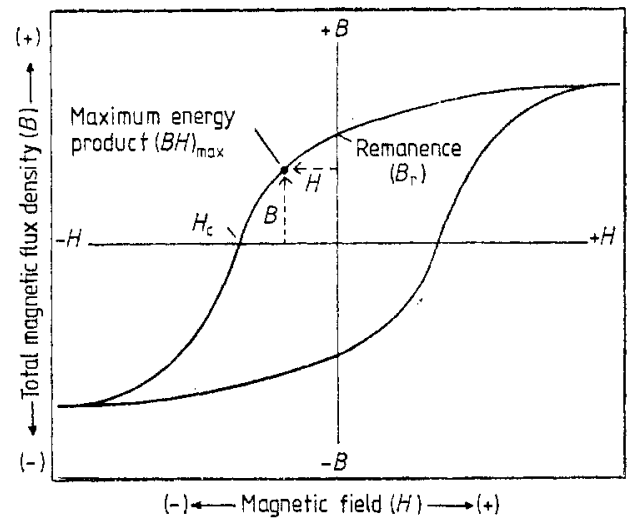

Figure 12. $B-H$ loop of a permanent magnet illustrating the point where the product $B-H$ is maximum. $B_{\mathrm{r}}$ is the remaining flux when $H$ is zero and $H_{\mathrm{c}}$ is the value of $H$ required to reduce $B$ to zero.

to zero is called the coercive force. Furthermore each point on the loop represents some value of $B H$. The point where this value reaches a maximum is known as the maximum energy product $(B H)_{\max }$. This maximum has been used as an index of quality for permanent magnets.

A field equal to the coercive force applied to Alnico magnets on the one hand and to the new rare-earth magnets on the other produces remarkably different results. Once the Alnico magnet has been driven to zero flux density the flux rebounds only slightly. With the cobalt-rare-earth magnets, however, whilst it is possible to drive the total flux density to zero, as soon as the coercive force is removed the flux density rebounds almost to its original value. The reason for this is that the cobalt-rare-earths have values for 'intrinsic coercive force' which are many times larger than their values for ordinary ferromagnetic materials. As a consequence a demagnetising field can drive the total flux density to zero or even below without affecting the intrinsic magnetisation $M$ of the material. This resistance to demagnetisation does not appear in the quantity 'maximum energy product' but it is equally important in the context of these new magnetic materials. Cobalt-rare-earth materials have values of intrinsic coercive force from 20-50 times greater than those of conventional permanent magnets. 
The property of the new rare-earth magnets to resist demagnetisation makes it possible to envisage many new applications and permanent magnet motors and alternators have already become established. In the context of the subject of this review repulsion forces between permanent magnets to support passenger carrying vehicles or trolleys capable of being moved about, say in factories, become technically feasible. If gaps between opposing poles of disc magnets are compressed to the same extent it can be seen from figure 13 (plate) that weights which can be supported by three magnetic materials, Alnico, barium-ferrite and cobalt-samarium are in the ratio of $2: 5: 23$. This means that if a 10 ton weight or vehicle is to be supported using ferrite magnets the total weight of the magnets, including those on the track as well as on the levitated body, would be nearly 2 tons. The weight of the levitated body or the vehicle would be 11 tons. If cobalt-samarium magnets were to be used the total weight of the magnets would be only about $\frac{1}{2}$ ton and thus the weight of the vehicle including the magnets would now be 10.25 tons.

\subsection{Permanent magnets for repulsion levitation}

A corollary of Earnshaw's theorem is that it is impossible for a body to be held in stable equilibrium against displacements in all directions if the system is constituted of permanent magnets only. When all the restoring forces are generated by repulsion between permanent magnets there remains at least one direction for which the body is in unstable equilibrium; the smallest displacement in this direction brings into being a force which tends to increase the displacement.

Complete levitation without any mechanical contact has been achieved by means of a combination of permanent magnets and electromagnets. Backers (1961) describes a magnetic journal bearing in which a shaft is supported with radial bearings of permanent magnet rings and support or axial location is provided by controlled electromagnets.

3.2.1. Levitation of vehicles and estimation of repulsion forces. Several advantages are claimed for vehicle systems using ceramic magnets in repulsion and electromagnetic or mechanical guidance systems. Some of them, such as absence of noise and vibration with reduced maintenance, are common to other suspension and levitation systems. Particular to ceramic magnet systems, however, are complete absence of the provision of energy to achieve lift, absence of induced eddy currents in the track since the track is non-conducting, thus obviating a drag force, and possibly lighter vehicles than with other schemes.

On the debit side it is doubtful whether a track consisting of permanent magnets can be constructed as cheaply as from mild steel rails even if modern magnetic materials can be produced in the large quantities required for track laying. It is just conceivable with barium-ferrite but barely so with cobalt-samarium and its descendants. The other factors on the debit side are related to the provision of guidance forces and forces for controlling the ride quality. As is suggested, any schemes which use controlled DC electromagnets would detract from the advantages claimed above as well as having to use two different technologies. Finally the problem of attracting debris to the track is probably far more serious and hazardous than so far indicated.

The Westinghouse Co. in America was the pioneer in this field and was the first to suggest the use of ceramic magnets for transport applications. A one-passenger vehicle was developed at Westinghouse Research Laboratories (Westinghouse Engineer 1965) in the early 1960s. The main proponent of such schemes in Great Britain has been 


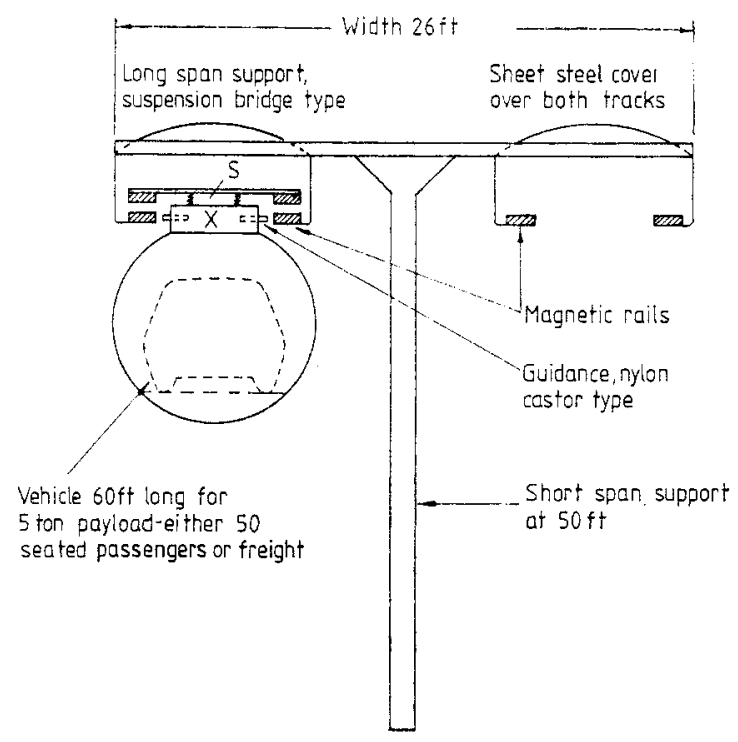

Figure 14. Polgreen's proposed rail configuration for vehicles using permanent magnets in repulsion. $x$, space for linear motor propulsion; $s$, secondary suspension for passenger comfort. (Reproduced by permission of J R Polgreen.)

Polgreen and figure 14 shows Polgreen's proposed rail configuration. This arrangement of rails with a steel backing is also favoured by Bahmanyar and Ellison (1974) as it is said to increase lift forces by better utilising the available flux and also provides better mechanical protection for brittle magnets.

3.2.1.1. Experimental estimation of forces. The calculation of the forces of repulsion between magnets is difficult and in general even when scaling up based on experimental results it may be subject to considerable errors. McCaig (1967) has outlined a method based on experimental results for a few configurations of Ferroba III magnets illustrated in figure 15 in which the repulsion force per unit area of the magnets is plotted against

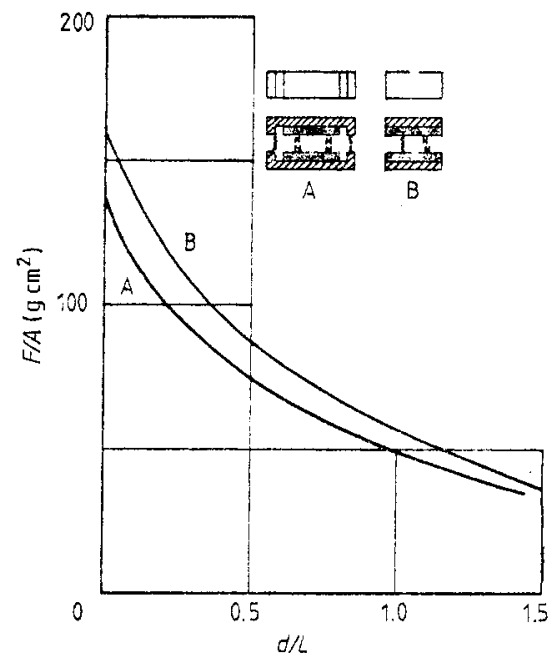

Figure 15. Repulsion forces between Ferroba III magnets. 
distance of separation divided by magnet thickness (called reduced distance). The repulsion force is defined as the total weight supported/magnet face area with faces separated by a distance $d$ at which the product force $\times$ distance is a maximum. The force increases with decreasing distance approximately so that it is doubled at $d / 2.4$ and quadrupled at zero.

The force of repulsion depends upon the magnet material, the arrangement of poles as described, the area, the shape and thickness of the magnets, the distance between the repelling pole surfaces and the dimensions of any steel parts used. Although these factors make the prediction of force difficult, if the same materials and configurations are used, a correct use of the method of similitude can enable the forces to be estimated for other systems in which all the dimensions and distances bear the same ratio to the one for which experimental results are available, since the distribution of magnetisation and polarity in the magnets should be the same. The force of repulsion should vary as the superficial area of the magnets, i.e. as $L^{2}$ where ' $L$ ' is any linear dimension. Since the volume, and therefore the mass, of the system varies as $L^{3}$, the force per unit mass varies as $L^{2} / L^{3}=L^{-1}$. It thus appears that for any material and arrangement there is a maximum size above which the magnets cannot levitate their own weight. This argument is valid if the distance between the magnets is increased as their size increases. In practice the distance of separation is more or less constant and increased loads can be supported by an increased number of magnets.

The weight of the supported magnet is subtracted from the force of repulsion in figure 15 , but not the weight of any steel parts. If, for a given pair of magnets, the force of repulsion, $F$, is measured for different distances of separation $d$ there is some value for $d$ for which the product $F d$ is a maximum. This may be considered the optimum condition for the given arrangement. The quantities $(F / A)_{\mathrm{opt}}$ and $(d / L)_{\mathrm{opt}}$ follow from this notion of optimal distance. Finally the quantity $F d / A L$ is a measured of the value of the product $F d$ per unit volume of the supported magnet.

The arrangements using mild-steel pole pieces are very efficient but in assessing the merits of different systems the weight of any mild steel in the support magnet must be taken into consideration. The cross section of the flux paths must also be sufficient not to cause losses due to saturation.

3.2.1.2. Analytical estimation of repulsion forces. The force of attraction between two magnets is given by the expression $F=B^{2} / 2 \mu_{0}$ per unit area. The flux density is assumed in this case to be uniform but when magnets are in repulsion this assumption is no longer valid. In fact, if two identical magnets were held in close contact the flux density would be zero but the force would be maximum. In order to arrive at the correct estimate it is necessary to consider the influence of one magnet on the magnetisation of the other. This may be possible by considering each magnet to consist of a distribution of poles, dipoles or current loops. McCaig (1961) has attempted an analysis of the forces of repulsion between small disc magnets by considering each to consist of a number of elementary charged layers. Alternatively, the same result could be obtained by treating each magnet as a charged surface. With these methods it is essential to evaluate the mean flux density or its gradient at various levels above the supporting magnet. It is necessary to use weighting functions to take account of the non-uniform fiald distribution in order to obtain satisfactory results. Bahmanyar (1973) claims to have circumvented these difficulties. It is assumed that the magnetisation vector is everywhere perpendicular to the pole face and that the upper part of the magnetisation curve is parallel to the $H$ axis of the $B-H$ graph. This is largely true for the modern high-coercivity-large-remanance 
ceramic magnets. The first step is then to compute the normal, i.e. the force-producing component of the flux density, $B_{z}$, due to the supporting magnet at every node of two $p \times q$ field matrices coincident with the two pole faces of the supported block. $p$ and $q$ are made as large as can economically be handled by a computer. Two directly opposite elementary meshes $\mathrm{ABCD}$ and EFGH on the upper and lower faces of the supported block are considered bound by rows $j$ and $j+1$ and columns $k$ and $k+1$ of the field matrix. Mesh length along the $x$ axis (parallel to the direction of motion) is $\delta R$ (figure 16) and along the $y$ axis it is $\delta P$. The gap length is assumed to be $h$ and the magnet depth $2 a$. Bahmanyar deduces that at a point height $z$ above the supporting magnet $B_{z}$ is given by

$$
B_{z}=\frac{M}{4 \pi} \iint\left(\frac{z}{\left(x^{2}+y^{2}+z^{2}\right)^{3 / 2}}-\frac{z+2 a}{\left[x^{2}+y^{2}+(z+2 a)^{2}\right]^{3 / 2}}\right) \mathrm{d} x \mathrm{~d} y
$$

and that the force of repulsion for this gap on the supporting magnet is

$$
F(h)=\frac{M P R}{\mu_{0}(p-1)(q-1)} \sum_{j^{\prime}=1}^{p-1} \sum_{k^{\prime}=1}^{q-1}\left[B_{z}\left(j^{\prime}, k^{\prime}, h\right)-B_{z}\left(j^{\prime}, k^{\prime}, h+2 d\right)\right]
$$

where $j$ and $k$ refer to the rows and columns of the symmetrically superimposed repulsion force matrix having nodes at the centres of each elementary mesh, $M$ is the intrinsic magnetisation and $r$ and $p$ are the dimensions of the magnet along the $x$ and $y$ axes, respectively. The last two terms in equation (3.2) are the mean flux densities over the elementary charged surfaces $\mathrm{ABCD}$ and $\mathrm{EFGH}$, respectively.

Bahmanyar has extended this analysis to magnetic track rail as well as the steel-backed structure similar to that proposed by Polgreen for Ferroba III (figure 17).

3.2.2. Cost of permanent magnet track. Some of the more general features of vehicle applications are considered later. These include problems of guidance, suspension stiffness and therefore ride comfort. However, these questions must be preceded by a question as to whether sufficiently large quantities of these ceramic magnets would be available to

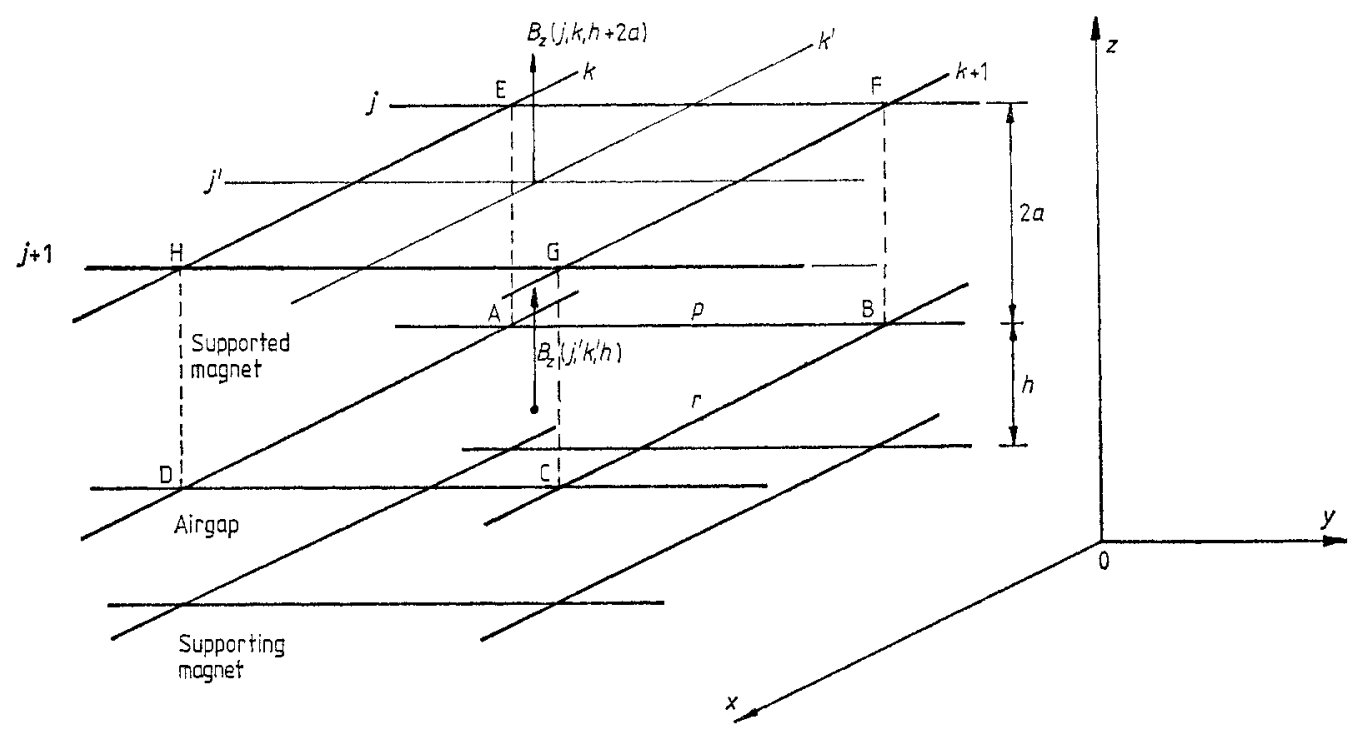

Figure 16. Model used for repulsion force analysis using the 'incremental mean flux density' technique of Bahmanyar (1973). 


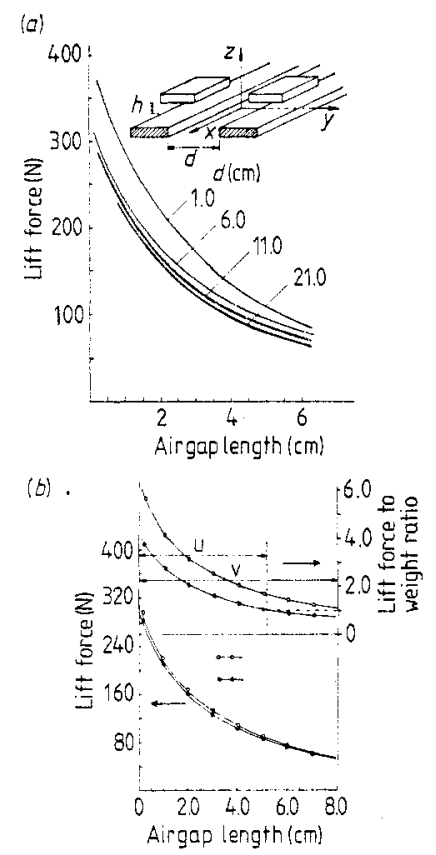

Figure 17. Lift forces in permanent magnet repulsion systems. (a) Lift force as a function of airgap length and rail spacing, $(b)$ lift force-airgap characteristics of steel channel backed magnetic rails. $G=$ gap between magnet sides and channel, $T=$ channel thickness. $O, G=3 \mathrm{~cm}, T=1 \mathrm{~cm}$; - $G=5.5 \mathrm{~cm}, T=1.5 \mathrm{~cm}$. u, v: ranges of load-carrying capacity. (Reproduced by permission of the IEE.)

build the track. Taking the example of the 10 ton vehicle and assuming that it will be $6 \mathrm{~m}$ long (as long as a single-decker bus) the weight of magnets per metre will be $35 \mathrm{~kg}$, i.e. 25 ton $\mathrm{km}^{-1}$ or 60 ton mile-1 of track. The price of samarium-cobalt is approximately $£ 1000 \mathrm{~kg}^{-1}$ but assuming that a cheaper version such as cerium-rare-earthcobalt magnets might cost $£ 250 \mathrm{~kg}^{-1}$ the cost of track magnets alone will be in excess of $£ 5000000 \mathrm{~km}^{-1}$, which does not seem prohibitive.

\section{Levitation using superconducting magnets}

There are, as indicated in $\S 2.3$, two ways in which superconductivity might be utilised to obtain levitation. The first is the Meissner effect, i.e. rejection of magnetic flux causing the superconducting body to behave as a perfectly diamagnetic body $\left(\mu_{r}=0\right)$. The second, which is the more practicable for application to vehicles, relies on the force of repulsion between a superconducting magnet moving on a conducting plate or guideway. This is the method proposed originally by Powell and Danby (1966). In a manner almost analogous to the development of permanent magnets leading to the interest and possibilities of transportation applications, the advances in superconducting materials has led to their consideration for high-speed vehicles. These advances are briefly reviewed before considering the levitation aspects.

\subsection{Some properties of superconductors}

The two properties mentioned in the previous section are the only ones of interest for the purposes of levitation. It is, however, also noteworthy in passing that superconductors 
form part of a category of materials at temperatures approaching $0 \mathrm{~K}$ called superfluids (London 1961). Two types of superfluids are known. One is represented by liquid helium (boiling point $4.2 \mathrm{~K}$ ) when cooled to $2.19 \mathrm{~K}$. The other type is represented by the superconducting state of electrons. Kammerlingh Onnes (1911), the Dutch physicist, discovered the latter phenomenon with mercury in 1911. Just as electric charge is transferred in a superconductor without a voltage difference between its ends, so superfluid helium can pass with ease through extremely narrow capillaries or fine cracks which would be impassable for any ordinary liquid. Furthermore, superfluid helium allows an extremely easy transfer of heat which has occasionally been described as 'heat superconductivity'.

Using his techniques of liquefaction of helium, Kammerlingh Onnes measured the electric resistance of various metals at liquid helium temperatures. He found much to his surprise that the resistance of mercury drops suddenly to an immeasurably small value when the temperature falls below a certain value (figure 18). This he determined to be about $4.2 \mathrm{~K}$, the so-called transition temperature of mercury. Kammerlingh Onnes called

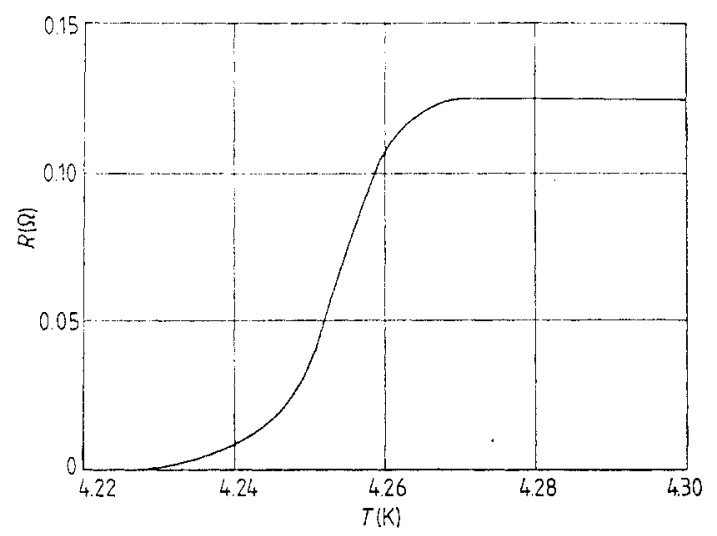

Figure 18. Resistance of mercury as a function of temperature.

this phenomenon superconductivity and further discovered that this property is destroyed when a strong magnetic field is switched on. He observed that there is a very sharp transition for a well-defined magnetic-field strength when the field is oriented parallel to the axis of the conductor and currents used for measuring resistance have low values. The field necessary to destroy superconductivity, the so-called critical or threshold field, depends on the temperature and disappears at the transition temperature with a finite slope. The curve representing the critical field $H_{\mathrm{c}}$ as a function of temperature (the threshold curve) has been measured for many superconductors (figure 19). It follows that if an electric current is caused to flow in a superconductor of such a value that the magnetic field due to it has a strength at the surface of the superconductor in excess of the critical value the superconductive condition will be destroyed. The transition between normal and superconducting states is reversible whether it is effected by changing the field strength or the ambient temperature.

In general, there will be a magnetic field, however weak, surrounding a superconductor which is being cooled below the transition temperature. As the superconductive condition spreads through the material, at transition, pockets of flux may in practice become isolated and cut off between advancing fronts of the superconducting regions. The flux in these pockets will be compressed between the advancing fronts to the critical value of 


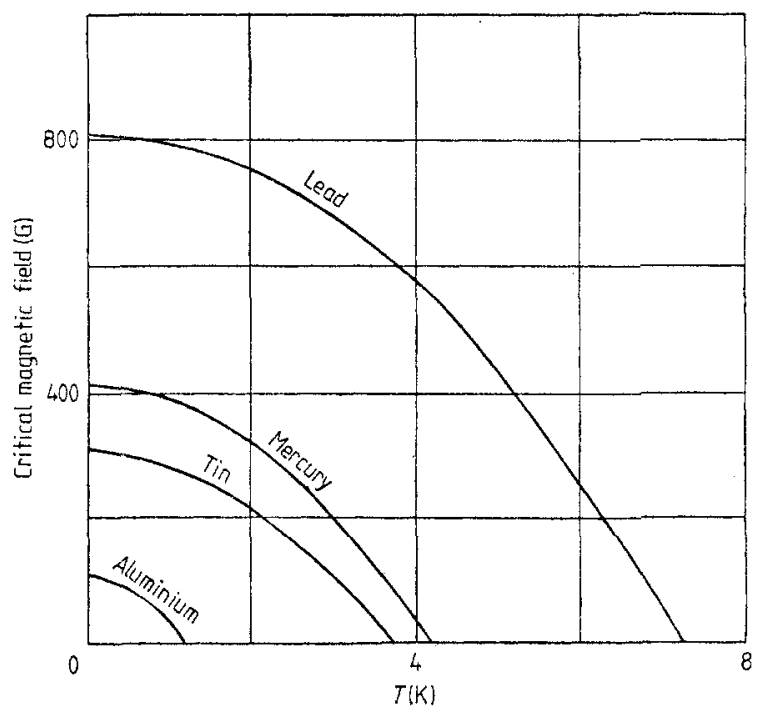

Figure 19. Critical temperatures and magnetic fields for superconductors.

the flux density; then the surrounding superconductivity, so to speak, cannot get into the pockets and the flux cannot get out. Thus it is difficult to obtain a superconducting body which is entirely free of magnetic moment although the amount of magnetic moment is likely to be of significance in components for special applications only, such as levitated superconducting gyro rotors.

The materials discussed so far, which include many of the elements (pure metals), are ideal or 'soft' superconductors. Practically speaking, transition with these materials and the exclusion of magnetic flux, apart from the frozen flux, is sudden and complete. Niobium and lead, which fall into this category, have high critical temperatures of $8 \mathrm{~K}$ and $7.5 \mathrm{~K}$, respectively, but niobium also has a high value of critical field of about 4000 Oe $\left(318 \times 10^{3} \mathrm{~A}\right.$ turns $)$. There are also non-ideal or 'hard' superconducting alloys and compounds which remain partly superconducting when penetrated by strong magnetic fields. They are being developed chiefly for the windings of superconducting electromagnets. Soft and hard superconductors differ in the manner in which they carry electric currents. In a soft superconductor current is carried only in a thin surface layer. In hard superconductors typical examples of which are compounds such as those consisting of vanadium and gallium $\left(\mathrm{V}_{3} \mathrm{Ga}\right)$ or niobium and tin $\left(\mathrm{Nb}_{3} \mathrm{Sn}\right)$ the current appears to be carried by filaments inside the material and the number of filaments can be increased by physically working the material. The effect of magnetic fields on a typical soft superconductor is also different from that on hard superconductors. When the field is low, under $0.1 \mathrm{kG}(0.01 \mathrm{~T})$, both conduct current in a thin surface layer and the field is excluded from the interior of the material. A moderate field of $1-10 \mathrm{kG}(0.1-1 \mathrm{~T})$ penetrates a soft superconductor, thereby destroying its superconductivity. In a hard superconductor, however, superconducting flow appears to be transferred to thin filaments and in certain hard superconductors filamentary flow persists even in high intensity fields of $10-100 \mathrm{kG}$ (1-10 T) and beyond, making them candidates for magnet windings of high-field modern superconducting magnets.

The synthesis of niobium-tin $\left(\mathrm{Nb}_{3} \mathrm{Sn}\right)$ was reported by Mathias (1957) as a compound that becomes superconducting at $18 \mathrm{~K}$ and remains even today one of the materials with 
highest transition temperature. It was pointed out by Kunzler (1961) that this compound satisfied the three essential requirements of a material suitable for the construction of superconducting magnets: (i) the material must remain superconducting in a high magnetic field; niobium-tin was found to be superconducting in a magnetic field of $88000 \mathrm{G}(8.8 \mathrm{~T})$, (ii) the material must sustain a high current density in a high magnetic field; niobium-tin was shown to sustain a current density in excess of $100000 \mathrm{~A} \mathrm{~cm}^{-2}$ at $88000 \mathrm{G}$, (iii) the material, even if refractory, must be capable of being fabricated into a magnet coil; niobium-tin is extremely brittle. However, if niobium-tin powder is enclosed in a niobium tube and then a monel metal jacket to act as an insulator and drawn through successive reducing dies it is formed into a wire which is wound directly on a former. The niobium-tin powder is converted into powder by heating the coil to a temperature of $1000^{\circ} \mathrm{C}$.

\subsection{Principles of superconducting levitation}

Reference was made in $\S \S 2.3$ and 2.10 to the two methods of using the superconducting phenomenon. The first is the Meissner effect to which considerable effort has been

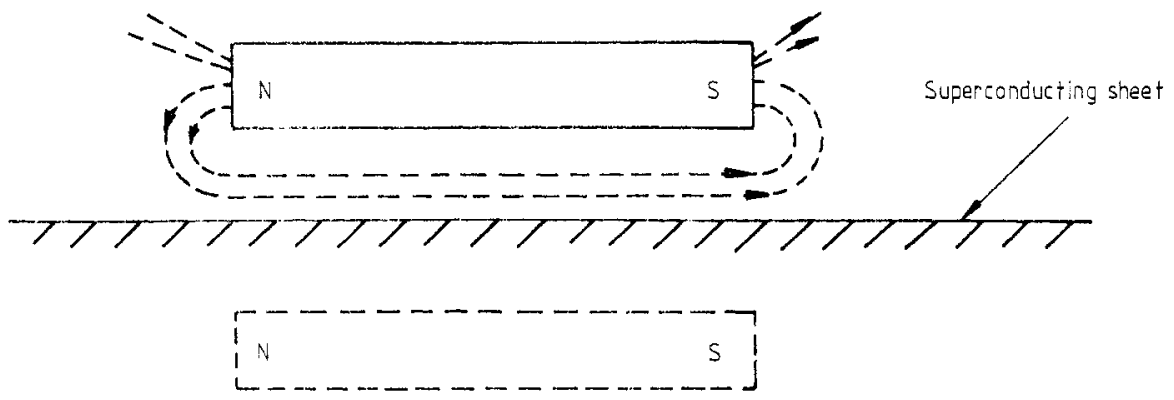

Figure 20. Levitation of a bar magnet on a superconducting sheet.

devoted in order to levitate gyro rotors. The second utilises forces of repulsion arising out of the interaction between a superconducting magnet and eddy currents induced in a conducting sheet as a result of relative motion between the two. This is the effect which seems the more practical for application to passenger carrying vehicles.

4.2.1. Levitation using the Meissner effect. When a bar magnet is brought near a horizontal superconducting surface we may regard the flux as being compressed into the space between the magnet and the magnetically impermeable surface of the superconductor (figure 20). Provided the compression of flux does not lead to the critical flux density being exceeded, the magnet will float on the superconducting surface in stable equilibrium in the vertical direction. If the surface is sufficiently large to be considered infinite in relation to the size of the magnet it will be in neutral equilibrium in the horizontal plane. It will, in effect, behave as if there is a mirror image of the magnet in the plane superconducting surface. When the size of the plane is reduced until the horizontal equilibrium becomes unstable stability can be restored by dishing the surface. The experiments of levitating a bar magnet having dimensions of several millimetres above a saucer-shaped superconducting surface were performed by Arkadiev $(1945,1947)$. There are reports of other experiments (Schoenberg 1960, Harding and Tuffias 1960, Buchold 1962) but Boerdijk (1956a, b) considered the possibility of levitating a magnet between an attracting 
magnet and a superconductor in the same way as he levitated a magnet between an attracting magnet and a piece of diamagnetic material.

Reported applications of this technique of using superconductivity to obtain levitation show that usually a superconducting object is levitated by flux from more than one magnet and that the levitated object is usually a rotor. The magnets are usually superconducting rings or coils which carry persistent currents. Simon (1953) reports theoretical and experimental investigations of the levitation of a superconducting hollow lead sphere $1.25 \mathrm{~cm}$ diameter and weighing $0.70 \mathrm{~g}$ over a pair of rings or coils carrying currents in opposite directions or ring permanent magnets with their axes vertical. Although Simon concluded that levitation was not possible over a single ring this was subsequently disproved by Harding and Tuffias (1960). This work, following the investigations of Culver and Davis (1957), was directed towards the development of the 'cryogenic magnetic suspension gyroscope'. Harding and Tuffias report the analysis of different configurations of superconducting current-carrying rings. They also describe other practical aspects such as the method for starting persistent current in the superconducting ring and the spot welding of niobium wire for making joints in the ring. Rotors were solid niobium spheres or lead plated by electrodeposition or vacuum deposition on to sapphire, plastic, aluminium or magnesium spheres. In the experiments described a $3.8 \mathrm{~cm}$ diameter lead-coated sphere weighing $8 \mathrm{~g}$ was levitated above a single ring carrying $1000 \mathrm{~A}$ turns. The largest sphere levitated was $4.25 \mathrm{~cm}$ diameter made of solid niobium weighing about $300 \mathrm{~g}$. Buchold (1962) also reports work on cryogenic rotor gyros at the General Electric Co. (USA). Construction of a cryogenic gyro with a niobium spherical rotor levitated between 10 coils of niobium wire carrying persistent currents is described. The rotors are typically $5 \mathrm{~cm}$ diameter with a wall thickness of $1 \mathrm{~mm}$ and weighing about $110 \mathrm{~g}$. An equatorial rim constitutes the armature of a cryogenic electric motor as the means by which the rotor is driven up to speeds of 40000-50000 RPM. The rotor has a radial clearance from its housing of just over $0.25 \mathrm{~mm}(0.12 \mathrm{in})$. The interior of the housing is evacuated and vent holes provided in the rotor wall. Other features such as sensing of rotor speed and attitude and refrigeration are also described. There is, in fact, a profusion of literature on cryogenic gyros and other devices emanating from the General Electric Co. (USA) around the 1960s.

The motion of a superconducting body levitated over a current-carrying superconductor is very oscillatory. It is, however, made considerably less so by locating a non-superconducting metal such as iron or copper near the current-carrying member. Changes in the distribution of flux around the superconducting ring or coil due to the approach and recession of the suspended body produce eddy currents in the nonsuperconducting metal and thus providing a means of dissipating energy.

4.2.2. Levitation using eddy currents induced by motion of superconducting magnets. This approach to magnetic levitation is based on the repulsion induced by a magnet travelling above a conducting surface. Conventional electromagnets or permanent magnets are also capable of induced levitation at small clearances but they are incapable of producing magnetic fields of the size and intensity required for anything heavy like, for example, a passenger carrying vehicle. Superconducting magnets due to their vastly greater magnetic fields have made this possible.

The phenomenon of electrodynamic levitation, as this phenomenon is now called, can be demonstrated very simply by spinning an aluminium drum at sufficiently high speed and placing a suitably (curved) shaped magnet attached to a flexible cantilever above it. The principle of this method of levitation is depicted diagrammatically in 


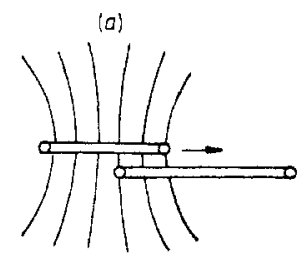

(b)

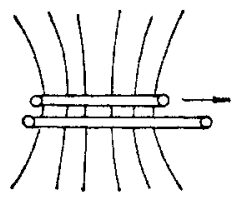

1c)

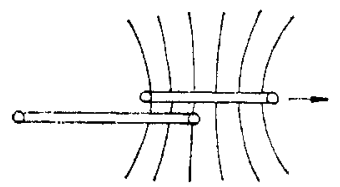

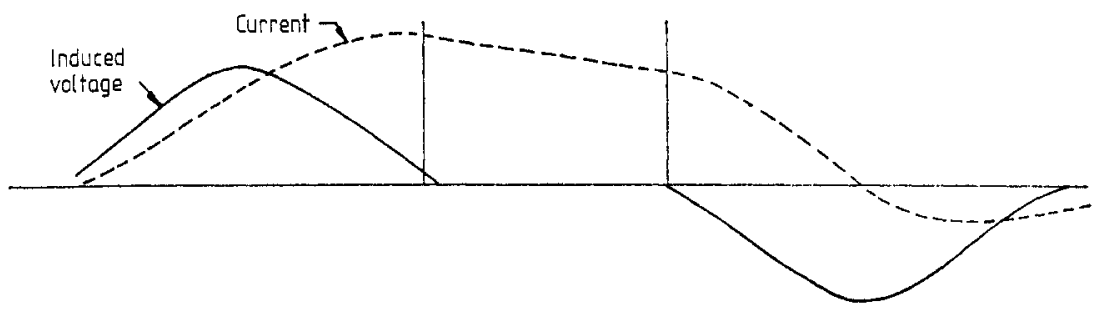

Figure 21. Principle of electrodynamic levitation systems.

figure 21. A current-carrying loop is assumed to be moving past a somewhat larger short-circuited loop. As the magnetic flux from the travelling loop begins to link the stationary loop it induces an EMF resulting in a current circulating in the loop and tends to oppose the change in the magnetic flux linking the stationary loop. The induced voltage is proportional to $\mathrm{d} \varphi / \mathrm{d} t$, i.e. to the rate at which the magnetic flux through the loop is changing, and the current is determined by the inductance and the resistance of the loop. When the moving loop is directly above the stationary loop the flux linking does not change and the induced EMF falls to zero. The induced circulating current begins to decay owing to resistive loss in the stationary loop. As the travelling loop begins to move away from the stationary loop the flux linkage begins to decrease and the induced EMF is now of a reversed polarity which not only brings the circulating current to zero but produces a current in the opposite direction that is equal to the amount by which the originally induced current has decayed. This residual current persists for a fraction of a second after the travelling loop (or the vehicle carrying this loop) passes and is called eddy current wake.

Both lift and drag are produced by the interaction of the two loops. Lift results from the fact that the induced current in the stationary loop flows in a direction opposite to the current in the approaching loop and therefore repels it. This repulsion alone would not produce a drag as its mechanical equivalent is a wheel passing over a hump in the road. All the energy lost in raising the wheel is regained when the wheel descends to its original level. Unfortunately, in the electromagnetic case the current in the stationary loop decays as the travelling loop passes and, therefore, not all the approach energy is recouped in the departure. The energy lost is, therefore, equal to the resistive dissipation in the stationary loop. The opposite current induced in the stationary loop due to decay is attractive and, therefore, causes drag. An unusual phenomenon is that the faster the vehicle carrying the current loops (which may be superconducting) travels the less the drag is. Decrease of drag with increasing velocity is rare in any transportation system.

All the early investigators thought in terms of a guideway composed of discrete loops or coils and there may in fact be an advantage in such arrangements. However, the loops can be replaced by continuous conducting sheets without any fundamental change in interaction. So long as the coil or the magnet is stationary the magnetic field is unaffected 
by a conducting but non-magnetic sheet. As the current-carrying coil begins to move eddy currents, which will be distributed in nature, are induced in the conducting sheet. Their overall effect is to generate a magnetic field which opposes that of the moving loop and to keep it from penetrating through the conducting sheet and in the process giving rise to lift forces.

The induced currents do not become strong enough to oppose the penetration of magnetic field through the sheet and to keep it out entirely until the current loop has reached a sufficiently high speed. Eventually when the current loop, if driven at an increasing speed, reaches this speed, virtually none of the magnetic flux is allowed to penetrate. The conducting sheet now behaves as a magnetic shield. The moving loop sees an image loop but of opposite polarity equidistant from the surface of the conducting sheet and thus repelling it. The force of repulsion becomes larger the smaller the distance of the moving loop from its image.

The main characteristics of electrodynamic levitation can be explained even with just these basic principles. If a current-carrying coil accelerates at a constant height above a conducting guideway it experiences a lift force at first proportional to the square of the velocity but eventually reaching a limiting value. The drag force is at first proportional to the velocity but then passes through a peak and decreases inversely as the velocity. At high speeds the drag decreases more slowly because of the skin effect. The induced eddy currents are largely confined to a thin layer near the surface of the conducting sheet and consequently the guideway appears to have higher resistivity. The electromagnetic drag, unlike the aerodynamic drag, decreasing with increasing speed is a remarkable feature of electrodynamic levitation. The lift force is proportional to the product of the perpendicular and parallel components (to the guideway) of the magnetic field and the drag is proportional to the square of the component of the magnetic field perpendicular to the surface of the guideway. The lift to drag ratio is, therefore, proportional to the ratio of the parallel component to the perpendicular component of the magnetic field. This relationship gives some insight into the way the configuration of the magnets on a vehicle can be optimised.

An interesting but very rough guide (which completely ignores the problem of overcoming the drag force) is that if the magnetic field at the surface is about $20 \mathrm{kG}(2.0 \mathrm{~T})$ sufficient force will be generated to just support the magnet and its notional payload at speeds as low as 20 mile h$^{-1}\left(32 \mathrm{~km} \mathrm{~h}^{-1}\right)$ and the limit of the lift force will be very nearly reached at $60 \mathrm{mile} \mathrm{h}^{-1}$ (approximately $100 \mathrm{~km} \mathrm{~h}^{-1}$ ). The lift force will be $60 \mathrm{lb} \mathrm{in}^{-2}$ $\left(4.2 \times 10^{4} \mathrm{~kg} \mathrm{~m}^{-2}\right)$, the same as the pressure in the tyres of a bus. It is, therefore, suggested that magnets about the size of the footprint area of a bus wheel tyre will levitate a bus; a suggestion which inspired the early investigators of a wheelless train.

\subsubsection{Some design considerations of passenger carrying vehicles with cryogenic magnets.} The principal considerations in the context of the electrodynamic system of levitation for passenger carrying vehicles are not only the geometry and configuration of the superconducting magnets but the propulsion schemes as well. Although the levitation system is capable of operating at large airgaps the linear induction motors require a much smaller gap. Current studies indicate that the linear motor and its control gear also represent a substantial fraction of the overall weight of the vehicle since the peak of the drag force occurs at speeds of approximately $10 \mathrm{mile} \mathrm{h}^{-1}$ and the thrust required to move past this is prohibitively high. At the upper end of the speed range collection of power of the order of several megawatts through sliding contacts would present very formidable problems. Additionally, ride quality studies indicate that a guideway surface equivalent 
to a poor road surface can be tolerated with the high clearance levitation but, without some form of active damping, the inherent oscillatory nature of the suspension will fail to satisfy the passenger comfort criteria. Not only does the lift mode need to be damped but also the lateral guidance. The problem of lateral guidance is indivisible from the design of the guideway. This discussion precludes any economic or environmental considerations of a new guideway alignment such as that envisaged for any advanced ground transportation scheme on the grounds that these will be similar for any new schemes irrespective of the technology.

4.2.3.1. Repulsion and drag force estimation. The generation of lift force with superconducting levitation schemes of this type is essentially a dynamic or speed-dependent phenomenon. There is, therefore, no lift force at zero speed and insufficient force until a certain speed is reached. Hence the vehicle must be supported in some fashion below such speeds and the commonly suggested solution is wheels. As the vehicle speed increases, for a magnet at constant height, the lift force $F_{L}$ increases rapidly at first and then levels off approaching the image force $F_{I}$ at high speeds (Borcherts et al 1973) (figure 22). $F_{\mathrm{I}}$ is the force between the coil and its image in the guideway at high speeds. As explained in $\$ 4.2 .2$ there is also the drag force $F_{D}$ on the moving coil as a consequence of the eddy current or Joule loss in the guideway. $F_{\mathrm{D}}$ also goes through a peak at about 10 mile $\mathrm{h}^{-1}$ and then drops off continuously as the speed is increased. The relatively low value of the magnetic drag at high speeds will add to the efficiency of this suspension (figure 23).
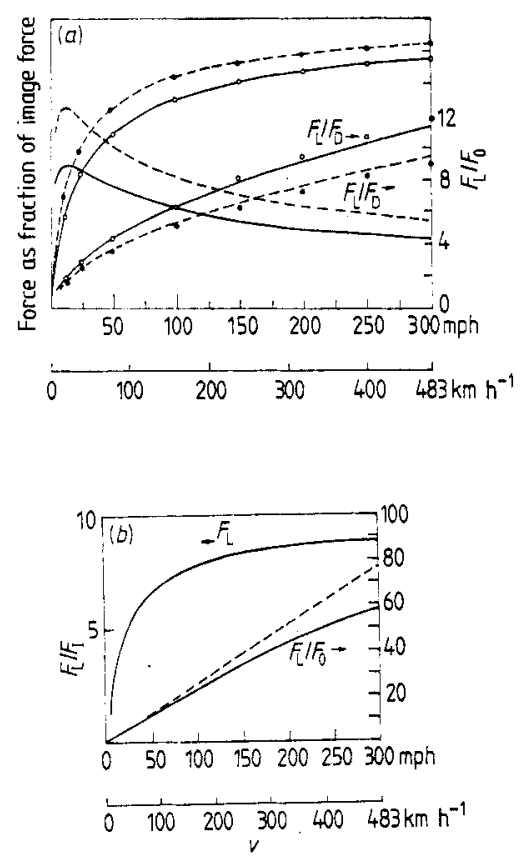

Figure 22. Drag characteristics for electrodynamic levitation systems. (a) Lift force $F_{\mathrm{L}}$, drag force $F_{\mathrm{D}}$ and lift to drag ratio on two rectangular coils above an infinitely thick aluminium slab as a function of speed. (The second dimension of the coils is in the direction of the motion.) - , theory, $O$, experiment: $2.12 \times 4.67 \mathrm{~cm}^{2}$ coil, $h=4.22 \mathrm{~cm}$. --- , theory, $\bullet$, experiment: $4.67 \times 2.12 \mathrm{~cm}^{2}$ coil, $h=4.22 \mathrm{~cm}$. (b) Lift force $F_{\mathrm{L}}$ and $F_{\mathrm{L}} / F_{\mathrm{D}}$ (lift to drag ratio) on a coil over an aluminium plate as a function of speed. $F_{I}$ is the image force. $0.5 \times 3 \mathrm{~m}^{2}$ coil, $h=0.3 \mathrm{~m}$, $2.54 \mathrm{~cm} \mathrm{Al}$ plate. (Reproduced by permission of the IEEE.) 

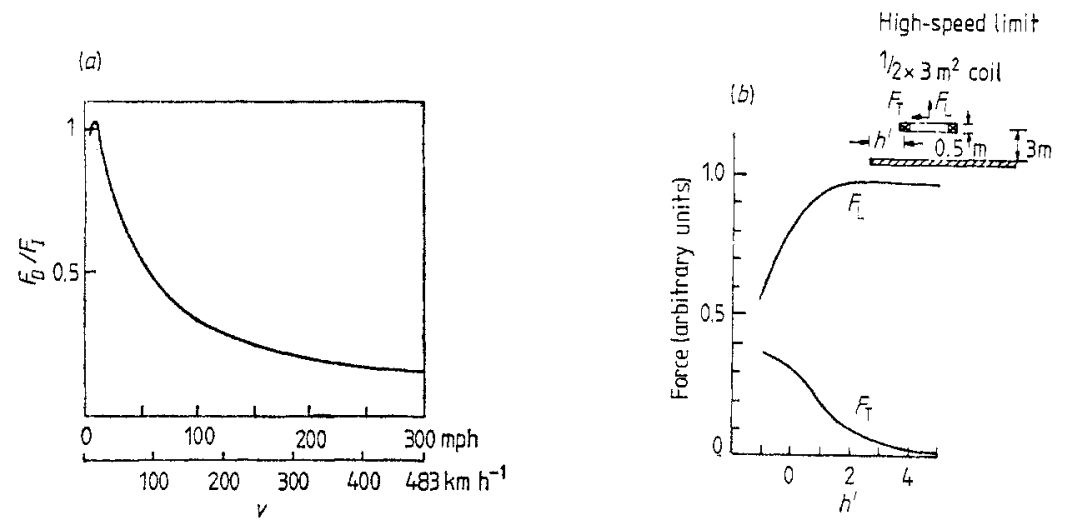

Figure 23. Lift and drag characteristics of electrodynamic suspension systems (cryogenic). (a) Drag force $F_{\mathrm{D}}$ as a function of speed, $(b)$ lift and transverse force on a coil near the edge of a conducting plate. (Reproduced by permission of the IEEE.)

The important parameters which characterise the suspension are lift and guidance forces per unit magnet weight and the $F_{\mathbf{L}} / F_{\mathrm{D}}$ ratio. Leaving aside the problem of guidance, calculations have been carried out (Reitz 1970, Reitz and Davis 1972) for rectangular coils moving with a velocity $v$ above a conducting plate of arbitrary thickness and infinite extent. An analytical fit to the calculations, if the plate thickness $T<\delta$, where $\delta$ is the skin depth of penetration and the permeability of the plate material $\mu=\mu_{0}$, i.e. non-magnetic, is

$$
F_{\mathrm{L}}=F_{\mathrm{I}}\left[1-\left(1-v^{2} / \omega^{2}\right)^{-n}\right]
$$

and (Davis 1972, Reitz et al 1972)

where

$$
F_{\mathrm{D}}=(\omega / v) F_{\mathrm{L}}
$$

$$
\omega=2 / \mu_{0} \sigma T
$$

$\omega$ has the dimensions of velocity and $\sigma$ is the conductivity. The number $n$ is determined by the dimensions of the coil. Equation (4.2) is an exact result. For the coil of figure $22(b)$, i.e. $0.5 \mathrm{~m} \times 3 \mathrm{~m} \mathrm{(} 3 \mathrm{~m}$ side parallel to $v$ ), $n$ is approximately equal to 0.2 and for aluminium plate 1 in $(2.5 \mathrm{~cm})$ thick $\omega=6300 \mathrm{~m} \mathrm{~h}^{-1}\left(3.9 \mathrm{mile} \mathrm{h}^{-1}\right)$. If the conducting plate is thick eddy currents are limited by skin depth $\delta$ and in equation (4.3) the value of $\omega$ is obtained by substituting $\delta$ for $T$ where $\delta=\gamma_{\mathrm{eff}} /\left(\pi \mu_{0} \sigma v\right)^{1 / 2}$ and $\gamma_{\mathrm{eff}}$ is the effective wavelength for the geometry under consideration (Reitz et al 1972). For a wide rectangular coil moving perpendicular to its length at a height $h$ small compared to its width, $\gamma_{\text {eff }}=16 h$. For a narrow coil, long side $2 b$, parallel to $v, \gamma_{\mathrm{epf}}=\frac{1}{2} \pi^{2} b$. For plates of intermediate thickness $F_{\mathrm{L}}$ and $F_{\mathrm{D}}$ have been determined numerically using Fourier methods. In general, it is found that increasing the levitation height and the length of the coil in the direction of motion improves the ratio $F_{\mathrm{L}} / F_{\mathrm{D}}$ but increasing the levitation height beyond $30 \mathrm{~cm}$ is not considered practical as the lift force diminishes. Figure 22(a) shows calculated and experimental values (Reitz 1970, Borcherts and Davis 1973) of $F_{\mathrm{L}}$ and $F_{\mathrm{L}} / F_{\mathrm{D}}$ for two coils, $2.12 \mathrm{~cm}$ and $4.67 \mathrm{~cm}$, at a suspension height of $4.22 \mathrm{~cm}$ over a thick aluminium plate. The experimental results were obtained by suspending the superconducting coils over the rim of a rotating aluminium wheel $61 \mathrm{~cm}$ diameter $\times 15.2$ $\mathrm{cm}$ width. The lift force on a $0.5 \mathrm{~m} \times 3 \mathrm{~m}$ coil at a suspension height of $0.3 \mathrm{~m}$ and $F_{\mathrm{L}} / F_{\mathrm{D}}$ 
are shown in figure $22(b)$. The drag force is shown in figure 23(a). Four such coils would support a 50 ton vehicle travelling at $v=483 \mathrm{~km} \mathrm{~h}^{-1}\left(300 \mathrm{mile} \mathrm{h}^{-1}\right)$.

The guideway plate acts very much like a 'thin' plate up to $160 \mathrm{~km} \mathrm{~h}^{-1}\left(100 \mathrm{mile} \mathrm{h}^{-1}\right)$. In this range it can be observed that $F_{\mathrm{L}} / F_{\mathrm{D}}$ is almost linearly proportional to speed and is essentially the same as that given by the thin plate analysis (broken line in figure $22(b)$ ). The eddy current distribution above $160 \mathrm{~km} \mathrm{~h}^{-1}$ is not uniform but is now limited by considerations of skin depth $\delta$ which is approximately $2 \mathrm{~cm}$ at $483 \mathrm{~km} \mathrm{~h}^{-1}$. The difference between the $F_{\mathrm{I}} / F_{\mathrm{D}}$ predicted by thin plate theory and the actual one is seen in figure 22 (b) to be almost $25 \%$ at $483 \mathrm{~km} \mathrm{~h}^{-1}$. Whilst theoretical calculations are based on rectangular geometry for the coil, in actual practice the corners will be rounded. This, however, does not have a significant effect on either $F_{\mathrm{L}}$ or $F_{\mathrm{D}}$.

There is in general a transverse force on the magnet if the guideway conducting plate is of finite width and the force is such as to push the magnet off the plate. Calculations and experiments show (Borcherts and Davis 1973) that if the track width exceeds the magnet width by about twice the levitation height the degradation in lift from that over an infinite plate is very small. The results from a $0.5 \mathrm{~m} \times 3 \mathrm{~m}$ coil for the lift force $F_{\mathrm{L}}$ and transverse force $F_{\mathrm{T}}$ for height above conducting plate $h=0.3 \mathrm{~m}$ by Borcherts and Davis (1973) are shown in figure $23(b)$. If the distance of the coil from the edge of the plate $h^{\prime}$ exceeds the suspension gap $h, F_{\mathrm{T}}$ is small and $F_{\mathrm{L}}$ is essentially the same as for an infinite plate. Since the drag force scales roughly with the sum of $F_{\mathrm{L}}$ and $F_{\mathrm{T}}$, the lift to drag ratio is degraded by about $10 \%$ if the track width exceeds the magnet width by only $2 h$.

It is necessary to have additional levitation surfaces in the guideway in order to provide transverse guidance forces for a suspended vehicle. The surfaces are preferably vertical ones and the vehicle can either have separate guidance magnets to operate against these surfaces or the main levitation magnets can operate in a dual role and provide guidance against the vertical sections of the guideway. The guidance force $F_{\mathrm{G}}$ generated by the lift magnet constituted by the same $0.5 \mathrm{~m} \times 3 \mathrm{~m}$ coil at levitation height of $h=0.3 \mathrm{~m}$ is shown in figure 24 . In either case the guidance force produces extra drag which degrades the overall suspension performance criterion given by $F_{\mathrm{L}} / F_{\mathrm{D}}$ since

$$
F_{\mathrm{D}}=(v / \omega)\left(F_{\mathrm{L}}+F_{\mathrm{G}}\right) \text {. }
$$

4.2.3.2. Cryogenic magnet design and cryogenic magnet requirements. The design of a magnetically levitated vehicle utilising superconducting magnets is crucially dependent upon a good magnet design which will transmit forces safely from the superconducting magnet elements, keeping the heat losses low and yet with minimum distance between the bottom of the Dewar and the superconducting wires.

The basic features of a cryogenic system for a vehicle operating in a U-channel guideway as suggested by Borcherts and Davis (1973) are shown in figure 25 . There are eight magnets, four for lift and four for guidance. This is not necessarily an optimum number. The lift and guidance pair are as shown in the figure at one of the corners and the arrangement allows a certain degree of redundancy, and hence safety, to be built into the system. The fringing field of the lift magnet can provide guidance in case of the failure of the guidance magnet and vice versa. In the example of the $0.5 \mathrm{~m} \times 3 \mathrm{~m}$ coil with $3.6 \times 10^{5} \mathrm{~A}$ turns the magnet and the Dewar assembly would weigh approximately $600 \mathrm{~kg}$ and hence give a lift to weight ratio of nearly 20 . In order to transmit the lift and guidance forces from the magnet at $4 \mathrm{~K}$ to the frame at $300 \mathrm{~K}$ some composite material such as epoxy fibre glass with very low specific conductivity but high tensile as well as compressive and fatigue strength must be used. Using layers of metallic reflectors to 


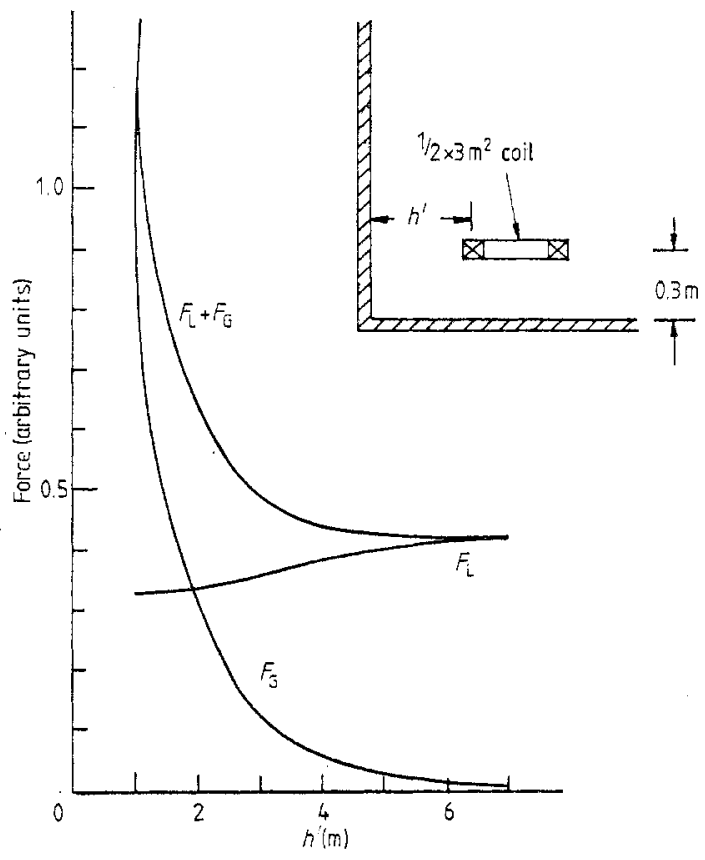

Figure 24. Lift and guidance force on a coil near a conductor shaped as a right angle corner. (Reproduced by permission of the IEEE.)
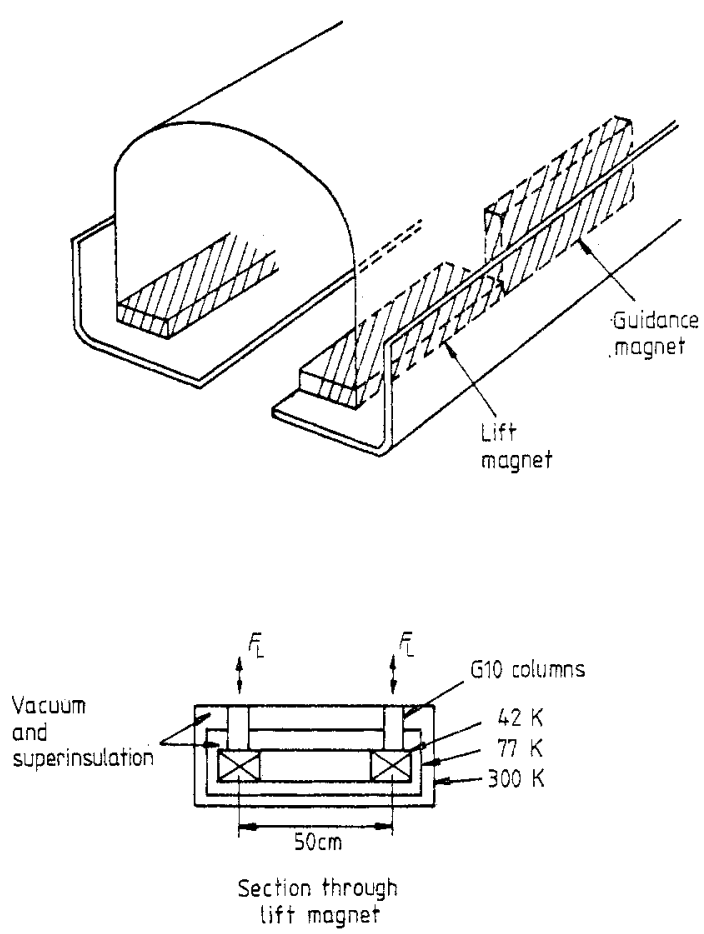

Figure 25. Basic features of a cryogenic suspension and guidance scheme operating in a U-shaped channel. (Reproduced by permission of the IEEE.) 
keep the radiation losses to a minimum, alternating with insulation spacers, the conduction and the radiant heat energy transmitted from $77 \mathrm{~K}$ to the magnet is $1 \mathrm{~W}$ for each.

A major source of heat leak into the cryostat is through the leads carrying the current in and out of the superconducting wires. For a well-designed pair of leads this would be approximately less than $2 \mathrm{~W}$ per $1000 \mathrm{~A}$ pair since the vehicle magnets are by no means large by present superconducting magnet standards. If the magnets are operated in the persistent mode these losses would be even further reduced. Typical figures of heat load to the $4 \mathrm{~K}$ cryostat would be less than $3 \mathrm{~W}$ per magnet or less than 24 per vehicle. There would in addition be AC losses in the superconducting windings due to the oscillatory motion of the magnets of the order of 1-10 W per magnet for the typical 10-12 $\mu \mathrm{m}$ diameter filaments in a multifilament niobium-titanium composite (Reitz et al 1972). The $4 \mathrm{~K}$ refrigeration for these magnets could come from on-board lightweight cryogenic refrigerators although such units have not yet been fully developed. The $77 \mathrm{~K}$ or the intermediate temperature refrigeration could be achieved by either liquid nitrogen, or the heat capacity in the boiled-off helium or an intermediate temperature point on the on-board refrigerator, although this would increase the size of the refrigerator.

4.2.4. Acceleration, braking and propulsion aspects of superconducting systems. The drag force on a magnet moving at constant height above a conducting track exhibits a peak (figure $23(a)$ ) in the speed range $8-16 \mathrm{~km} \mathrm{~h}^{-1}\left(5-10 \mathrm{mile} \mathrm{h}^{-1}\right)$ depending upon coil geometry and the thickness of the conducting track. At high speeds the drag falls off inversely as the speed, i.e. if the vehicle remains on wheels up to some lift-off speed $v_{0}$, the magnetic drag on the vehicle is similar in shape to figure $23(a)$ provided $v_{0}>80 \mathrm{~km} \mathrm{~h}^{-1}$ and the magnets remain at constant height for $v<v_{0}$. For a 50 ton vehicle the estimated magnitude of the low-speed drag peak is $8.9 \times 10^{4} \mathrm{~N}(20000 \mathrm{lb})$ inclusive of the drag associated with the guidance magnets (approximately half of the lift forces). The total drag force on such a vehicle due to both aerodynamic and electromagnetic forces is shown in figure 26 . This force is calculated on the assumption that the vehicle is entirely

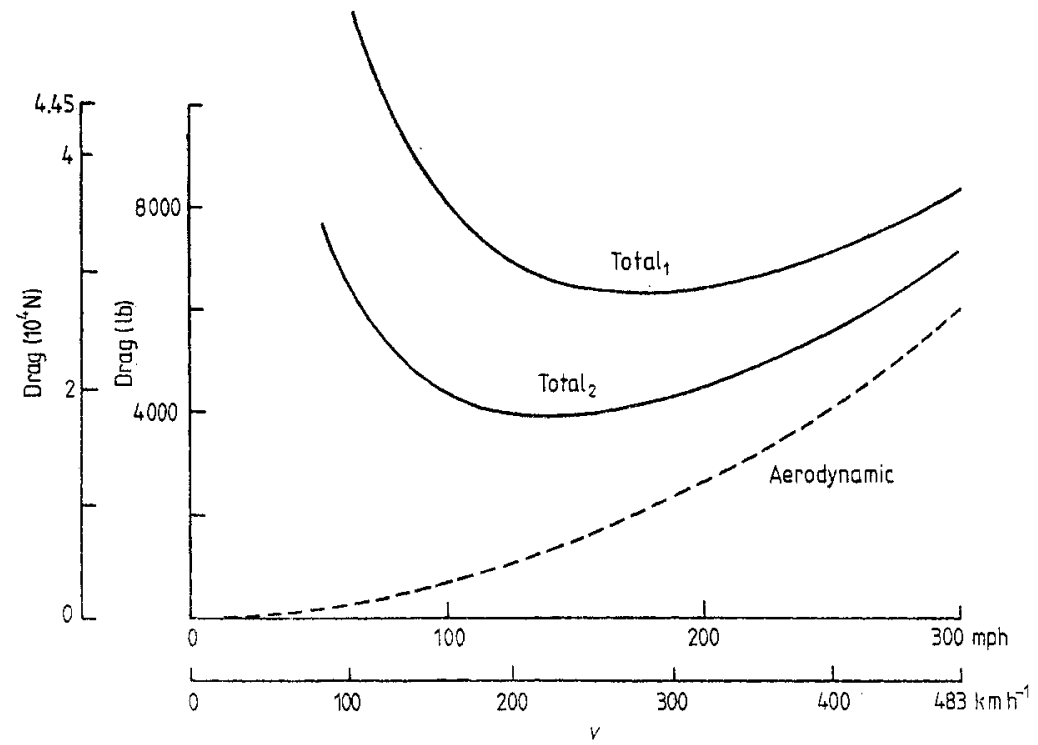

Figure 26. Propulsive force requirements to overcome magnetic and aerodynamic drag as a function of speed. (Reproduced by permission of the IEEE.) 
levitated. The aerodynamic drag is proportional to $v^{2}$ and at $483 \mathrm{~km} \mathrm{~h}^{-1}$ is estimated (Reitz et al 1972) to be $2.7 \times 10^{4} \mathrm{~N}(6000 \mathrm{lb})$. Two cases are illustrated in figure 26 , one for $F_{\mathrm{L}} / F_{\mathrm{D}}=40$ at $483 \mathrm{~km} \mathrm{~h}^{-1}$ and the second one for $F_{\mathrm{L}} / F_{\mathrm{D}}=80$ at $483 \mathrm{~km} \mathrm{~h}^{-1}$. For the case of $F_{\mathrm{L}} / F_{\mathrm{D}}=40$ the magnetic drag at $483 \mathrm{~km} \mathrm{~h}^{-1}$ is approximately $1.5 \mathrm{MW}$ and the aerodynamic drag is $3.5 \mathrm{MW}$ giving a total of nearly $5 \mathrm{MW}$. The magnetic drag at $80 \mathrm{~km} \mathrm{~h}^{-1}$ for this case, however, is $6.7 \times 10^{4} \mathrm{~N}(15000 \mathrm{lb})$. It would thus appear that the drag is appreciable over the entire speed range. There will, therefore, be considerable problems in accelerating the vehicle through this drag peak with a thrust-limited engine such as a linear induction motor.

A possibility is to look at ways of reducing the drag peak. Methods suggested for reducing or eliminating the low-speed drag are (i) not to operate the magnets in a persistent mode but to reduce the current during acceleration. The magnet coils, however, will possess substantial inductance and it could take as much as $10 \mathrm{~s}$ to bring the current up to full value. It would also introduce some additional cryogenic losses. (ii) Conductor to be left out of the initial section of the guideway and in order to avoid an abrupt increase in the drag force the conducting track to be brought up to the wheel level gradually from below. (iii) By tapering the aluminium plate in the track although the drag peak cannot be avoided this way. However, the vehicle can pass through the drag barrier of a tapered section if it has sufficient velocity. Thus the vehicle could be accelerated to $80 \mathrm{~km} \mathrm{~h}^{-1}$ at first in a guideway without a metal plate, then, with a section having tapered plate limited to $15-30 \mathrm{~m}$ in length, the reduction in speed in passing through the drag peak will be only $1.5-3 \mathrm{~km} \mathrm{~h}^{-1}$. (iv) The vehicle is fitted with wheels which maintain the magnets at a height greater than the operating gap. All the electromagnetic forces including the drag peak are thus reduced. When the vehicle reaches a speed substantially above the lift-off or the drag peak speed the wheels are retracted. Although this scheme adds the mechanical complexity of retractable wheels it has the advantage of being able to operate anywhere on the guideway so long as provision is made for wheeltracks. (v) A refinement of the superconducting guideway proposed by Powell and Danby is known as the 'null flux' method. Two opposing guideway loops are arranged flanking a single coil on the vehicle or two vehicle loops are arranged flanking a single guideway loop (figure 27). This results in an extremely high field gradient and a significant reduction in drag. A null flux system not only provides a high lift to drag ratio but also strong restoring forces. The suspension stiffness, in fact, is so high that guideway alignment might become critical and the ride uncomfortable without secondary suspension. The secondary suspension might add considerable weight and create additional aerodynamic drag and could thus cancel out much of the advantage. Based on the magnetic and aerodynamic drag calculations estimates of peak acceleration range from $0.17-0.3 \mathrm{~g}$ depending on the coil geometry and $v_{0}$. Peak deceleration is reduced by either increasing $v_{0}$ or by increasing the length/width ratio of the coils. Increasing the thickness of the conductor in the guideway is equivalent to increasing $v_{0}$ since it is $v_{0} / \omega$ which is important. TACV specifications of normal deceleration not exceeding $0.15 \mathrm{~g}$ and emergency deceleration of not more than $0.3 \mathrm{~g}$ can, therefore, clearly be met.

4.2.4.1. Linear synchronous motors with superconducting magnets. There is a common belief that a linear induction motor with the primary on the vehicle is the only possible means of propulsion unit viable for advanced ground transportation. In the case of vehicles levitated by superconducting magnets the problems of the drag peak and operation at airgaps of the order of $0.1-0.3 \mathrm{~m}$ pose a serious question mark against the linear induction motor. The linear synchronous motor was at first ignored because of the 


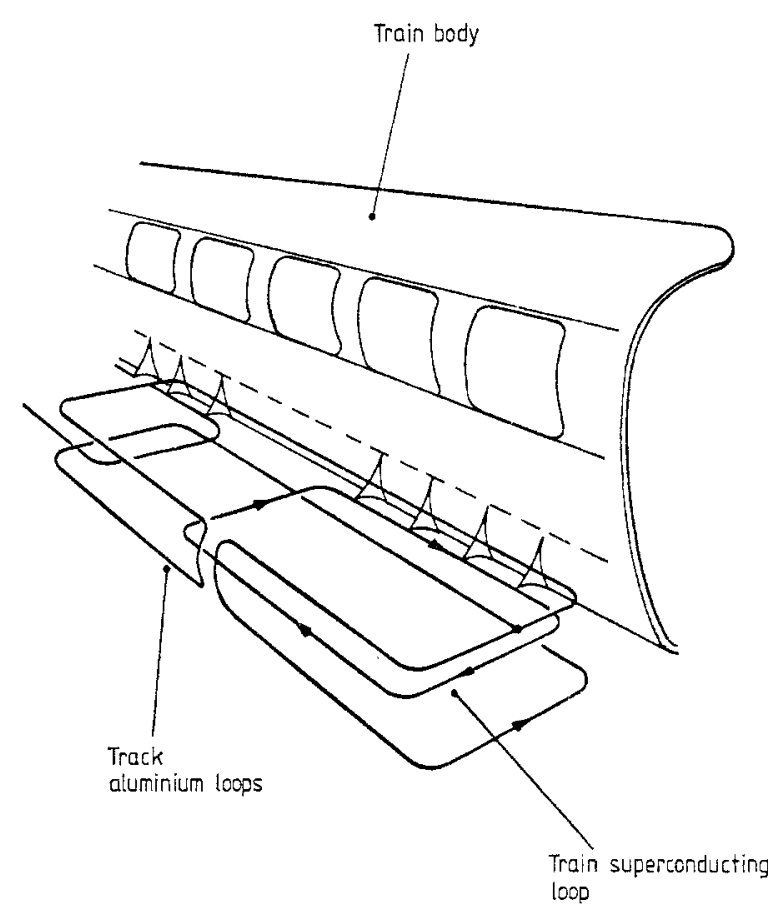

Figure 27. Null flux suspension proposed by Powell and Danby (1966).

requirement of synchronisation between the speed of operation and the frequency of its supply. It was also assumed that the track structure, particularly the long stator, i.e. a wound track fed from inverters, would be prohibitively expensive. The advent of superconductivity and advances in inverter technology have changed the situation so completely that the linear synchronous motor may now be the key to propulsion at high speeds. The superconducting levitation magnets provide fields that are not only intense but of large volume so that they can be coupled efficiently to fixed stator windings wound directly in the guideway with no iron, even at clearances of the order of $0.1-0.3 \mathrm{~m}$. The increase in the rotor current, i.e. the on-board superconducting magnets, opens up the way to a reduction in the stator currents and so to simpler and less costly stator structures than possible hitherto. As to variable frequency inverters or converters the recent advances in power transistor technology have opened up the possibilities of light, compact, efficient and also reliable variable frequency sources alongside the track. The higher the superconducting coil currents the lower the track currents with correspondingly high efficiencies. Typical cruise efficiencies are of the order of $80 \%$ depending on the length of the superconducting loops, the amount of current carried and the length of the energised track. If the currents in the superconducting coils are as large as $3-5 \times 10^{5} \mathrm{~A}$ track currents may be as low as 1000-1500 A and a thrust in excess of $8.9 \times 10^{4} \mathrm{~N}(20000$. 1b) appears feasible for clearances of $0.1 \mathrm{~m}$.

4.2.5. Current activity in superconducting levitation. The two most advanced developments, currently, are those of the Japanese National Railways (JNR) and the Canadian Institute of Guided Ground Transport (CIGGT). As indicated in $\$ 2.3$ JNR have built two vehicles so far (Yamamura 1976). The first vehicle was $7 \mathrm{~m}$ long, $2.5 \mathrm{~m}$ wide, $2.2 \mathrm{~m}$ 
high and weighed 3.5 tons. It was propelled by a linear induction motor which had its primary on the track and secondary on the vehicle. The track was only $480 \mathrm{~m}$ long and hence the maximum speed achieved was limited to $65 \mathrm{~km} \mathrm{~h}^{-1}$. The clearance at this speed was $6 \mathrm{~cm}$ and lateral guidance was provided by wheels operating against side rails.

The second JNR vehicle illustrated in figure 3 is being tested on a much longer track of $20 \mathrm{~km}$ and was finished in 1978 . Therefore, it is a little too early to obtain any published results for its operation. It is $10 \mathrm{~m}$ long, $3.8 \mathrm{~m}$ wide, $2.7 \mathrm{~m}$ high and weighs 10 tons. The lifting superconducting magnets have $1.5 \times 10^{5} \mathrm{~A}$ turns each and are installed horizontally on the vehicle. The secondaries are aluminium coils on the guideway and the configuration adopted is in the form of a ladder track. The lifting magnets act in the 'normal flux' mode. Another set of superconducting magnets, each with $4 \times 10^{5}$ A turns, is installed vertically on the vehicle and this set of magnets produces thrust and guidance forces acting on vertical coils attached to the guideway. The guidance magnets act in the 'null flux' mode. The vertical coils are also the primary of the linear synchronous motor and are energised by a cycloconverter in the frequency range $0-33.3 \mathrm{~Hz}$. Synchronisation of the LSM is maintained by optical means.

Studies and some experimental work are in progress in Canada (CIGGT) into electrodynamic levitation and linear synchronous motor propulsion (Eastham 1975). The experimental work consists mainly of a wheel $7.6 \mathrm{~m}(25 \mathrm{ft})$ in diameter rotating about a vertical axis at peripheral speeds up to $101 \mathrm{~km} \mathrm{~h}^{-1}\left(63 \mathrm{mile} \mathrm{h}^{-1}\right)$ driven by a $1750 \mathrm{RPM}$ $120 \mathrm{~kW}$ variable speed DC motor through a $25: 1$ reduction gearbox. Guideway components can be attached to the vertical rim while vehicle-borne components are mounted in a stationary harness and six component balances are used for positional adjustment and for the measurement of forces and torques.

The Canadian investigation is aimed at developing vehicles with linear synchronous motor propulsion and guidance for $480 \mathrm{~km} \mathrm{~h}^{-1}$ intercity transit along the TorontoOttawa-Montreal corridor for the 1990s. The vehicles are intended to carry 100 passengers, weighing 30 tons and levitated with $15 \mathrm{~cm}$ clearance by eight vehicle-borne superconduct-

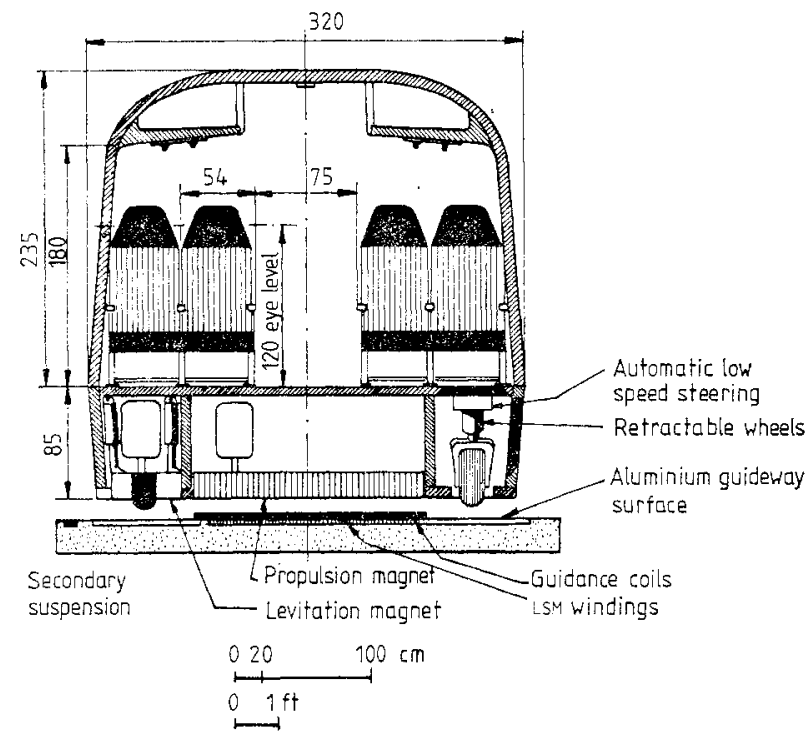

Figure 28. A schematic cross section of the proposed Canadian vehicle using superconducting levitation and guidance. All dimensions are in $\mathrm{cm}$. (Reproduced by permission of the CIGGT.) 
ing magnets (Eastham 1975, Eastham and Atherton 1975). The lift magnets are $1.0 \mathrm{~m}$ long $\times 0.3 \mathrm{~m}$ wide with $3.85 \times 10^{5} \mathrm{~A}$ turns interacting with eddy currents induced in $80 \mathrm{~cm}$ wide aluminium strips on a flat-topped guideway. The aluminium strip is graded from $1 \mathrm{~cm}$ at high-speed to $3 \mathrm{~cm}$ at low-speed sections to maintain the total drag (magnetic and aerodynamic) almost independent of speed whilst minimising the combined costs of energy requirements and aluminium amortisation. For the synchronous propulsion system the vehicle is supposed to carry 50 superconducting magnets each $0.4 \mathrm{~m}$ long $\times 1.5 \mathrm{~m}$ wide and the guideway is to have split three-phase windings energised by variable frequency current source inverters in $5 \mathrm{~km}$ sections to give $72 \%$ efficiency and 0.73 power factor. The thrust force is controlled by sensing the phase angle between supply current and the guideway windings, thus minimising the inverter rating and introducing the possibility of dynamic control of the vehicle.

A flat-topped guideway is proposed to minimise ice and snow accumulation and a new technique is proposed for obtaining lateral guidance by using interaction of the 50 propulsion magnets with the edges of the levitation strips and with null flux loops overlaying the LSM windings. The stiffness expected to be obtained is $10^{6} \mathrm{~N} \mathrm{~m}^{-1}$. The outline of the proposed vehicles is shown in figure 28 .

\section{Levitation using eddy currents induced by mains frequency excitation}

Conducting materials in solid and liquid states can be levitated above AC coil systems. The levitation of solid plates (Laithwaite 1965) or rings has been used as a laboratory or lecture demonstration. The levitation of molten metal for zone refining (Orkress et al 1952) dates back to the early 1950 s when there was considerable activity in the general area of induction machines. The notion that this form of levitation could be applied to passenger carrying vehicles appears to have originated towards the end of the 1960 s or early 1970s (Laithwaite et al 1971, Eastham and Laithwaite 1974, Laithwaite 1973a) as a result of several years of study related to the use of linear induction motors as propulsion units for high-speed vehicles (Barwell and Laithwaite 1967, Chirgwin 1974). The idea that linear induction motors could be used not only to propel passenger carrying vehicles but to levitate them as well gained considerable attention and popularity in the mid1970s. The idea became known as the 'magnetic river'.

A single-sided linear induction motor can be designed to produce large levitation forces in addition to its normal translational or tractive force. As the speed of the linear motor with respect to a composite reaction plate, consisting of conducting material backed by permeable material such as steel, increases, the force of repulsion becomes a force of attraction in normal design. The concept of magnetic rivers revolves around the objective of the design of a single-sided linear motor which could combine the functions of propulsion, levitation and guidance. Furthermore, in such a motor the force of repulsion would remain constant up to running speed with little or no additional input.

\subsection{Some stable and unstable $\mathrm{AC}$ induction levitators}

The study of AC levitation is closely linked with that of induction machines. For example, the lifting force on a conducting sheet over the surface of a single-sided linear motor can be calculated (West and Hesmondhalgh 1962) provided the equations for the flux density $b$ and the current density $j$ at all points can be found. However, in most practical levitation systems the equations either cannot be formulated or are so formidable that solu- 
tions are virtually impracticable. A qualitative appreciation based on an experimental approach is likely to be more fruitful.

A jumping ring experiment (figure 29) is an example of forces of repulsion between two current-carrying loops threaded by an open magnetic core. The geometry lends itself to fairly accurate calculations of force if the leakage flux is assumed to decay exponentially (West and Jayawant 1962). Until recently, when an application was proposed for this in the aluminium smelting process for stirring molten metal (Bamji 1974), this experiment was not much more than a good lecture demonstration.

If the iron core in figure 29 is sufficiently long and the ring allowed to take up a steady-state position as shown in the figure, it will be found that the ring will always be in contact with the core somewhere along its perimeter. The ring cannot, therefore, be considered as levitated stably since it is restrained by the core. The link between the study of conventional motors and their driving forces on the one hand and the study of levitation using AC and lift forces on the other is provided to some extent by the shaded

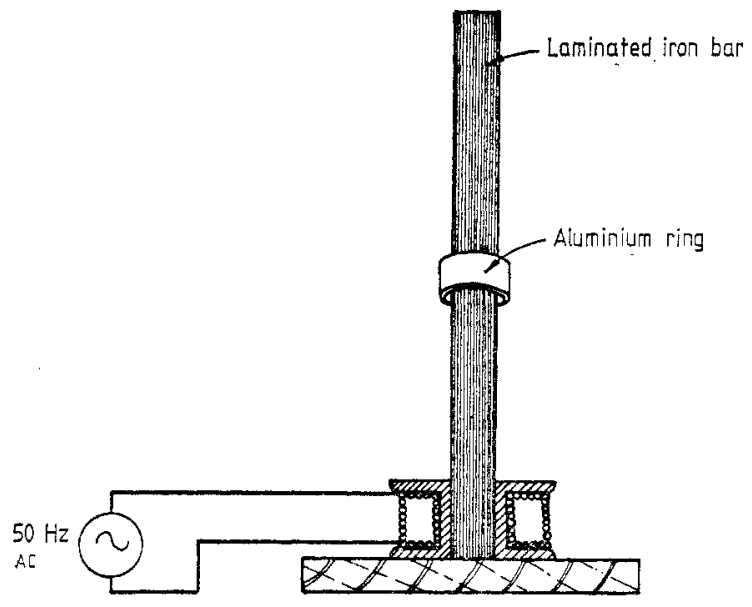

Figure 29. Jumping ring experiment.

pole motors (Laithwaite 1965). In such motors the phase changes produced by the shading ring are capable of producing force on the ring itself. The transition from a conventional three-phase induction motor to a circular plate levitator through a shaded pole motor and a ring levitator (Laithwaite 1965, 1966) is shown in figure 30. Starting with a conventional three-phase motor in $(a)$ in which the phase progression of the airgap flux along the perimeter of the rotor is dictated by the voltages to which the stator windings are connected, the first step is to replace the polyphase winding by a single-phase winding with shading ring(s) on the poles $(b)$. Next the rotor conductors are replaced by a continuous cylinder and the machine unrolled into a linear machine $(c)$. It is then rerolled about the axis $\mathrm{AB}(d)$ with the stator winding on the inside. The thrust is now such as to push the conducting cylinder out of the stator. The motor is then turned through $90^{\circ}$ so that the force on the cylinder is now vertically upwards. The length of the machine is now cut down so that the number of windings and the shading rings is reduced to the minimum $(f)$. The next step shown in $(g)$ is the important one of using the shading ring to produce a force on itself. The jumping ring geometry is one in which the outer iron cylinder is removed. The action is still one of a travelling field moving up the annular slot. The flux fringes above the slot opening and thus the action does not 

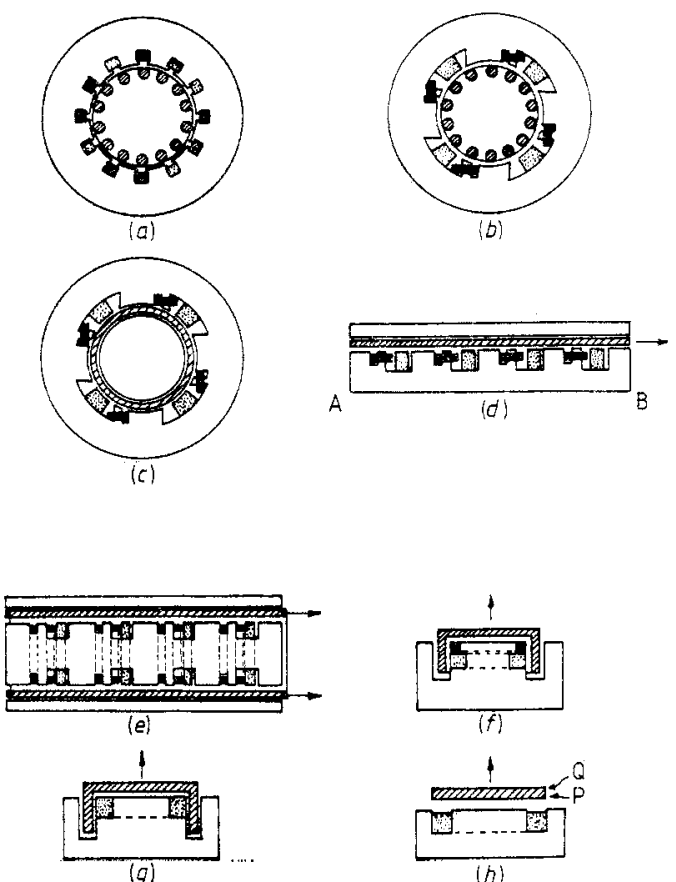

Figure 30. Topological steps from a three-phase squirrel-cage motor to a levitation system. (a) Conventional rotary machine, $(b)$ shaded pole rotary machine, $(c)$ shaded pole drag cup machine, (d) linear shaded pole motor with sheet rotor, $(e)$ tubular shaded pole motor with axial flux, $(f)$ single coil tubular shaded pole motor, $(g)$ elementary levitator, $(h)$ circular plate levitator. (Reproduced by permission of the IEE.)

stop abruptly there. Also, therefore, phase changes of current and flux occur in the parts of the cup outside the slot. Currents circulate around the rim of the cup and ideally there are no currents in any other axis. The cylindrical rim of the cup is now removed leaving a circular conducting ring, the hollow part of which may now be filled $(h)$, thus completing the transition to a circular plate levitator. The currents which circulate around the lower part of the periphery in position $\mathrm{P}$ are in phase advance over those circulating around the upper part in position $\mathrm{Q}$, and the lifting force may be attributed to a travelling field pattern travelling upwards and operating on the plate in the manner of an induction motor. It is relatively simple to show that the system is unstable. As soon as the plate is displaced slightly from the central axis (figure 31 ) the forces originating from the pole shading action will be greater on the overhanging side than on the other and the plate will be thrown off the coil-core face. The reason for the disc being thrown off may also be attributed to the presence of the plate producing radially outward travelling fields. Stability in principle could be achieved using a number of concentric circular coils each connected to voltages of different phase and thus producing fields travelling radially inwards. In practice, two such coils have been found sufficient to produce stability. The core may be made up of radial laminations, the tapered form achieved by staggering them radially (figure $32(a)$ ). More conveniently it could be made into blocks as shown in figure $32(b)$. Stable suspensions can be obtained for a range of plate diameters between the outer diameter of the inner exciting coil and the inner diameter of the outer exciting coil. The conditions of stability vary widely with the diameters, even within this range. Plates of diameters less than the outer coil diameter can be suspended stably with their 

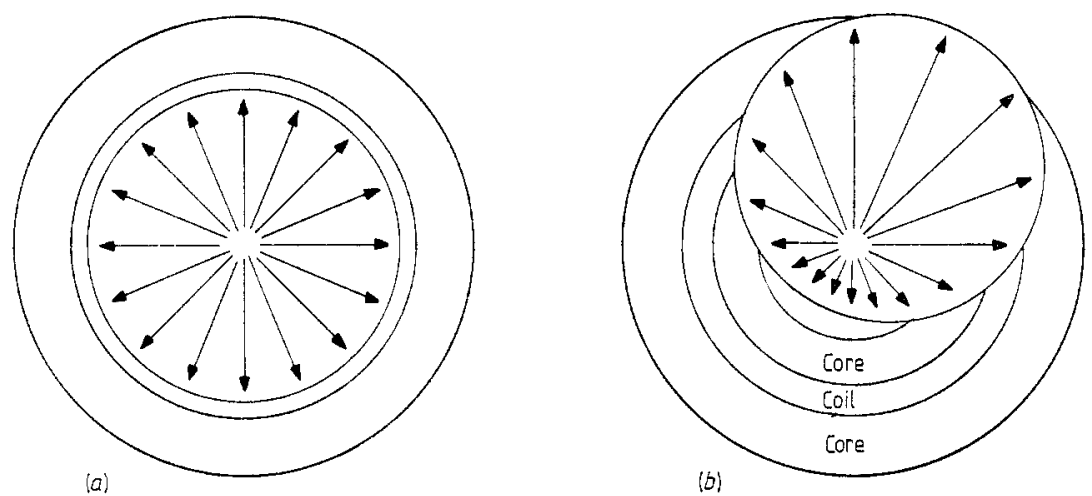

Figure 31. Horizontal forces produced by a circular plate levitator. (a) Plate concentric, (b) plate eccentric. (Reproduced by permission of the IEE.)

centres displaced away from the centre of the coil. The plate is then also capable of being spun as if it were inside an invisible tube of a larger diameter than that of the plate itself. The principal design dimensions given by Laithwaite (1966) for a circular plate levitator are height $5 \frac{1}{2}$ in, overall diameter 8 in with the lamination width $\frac{1}{2}$ in; the number of turns on the inner coil 480 and the number of turns on the outer coil 440 of 15 SWG enamelled copper wire.

The height of the plate above the coil for any particular value of the inner coil current does not vary a great deal when the outer coil current is in the range where the levitated plate is stable. The formula given by Laithwaite (1966), based on the assumption that for a given height the upward force on the plate is proportional to the square of the primary current, is lift at height $h$ is proportional to $1 /(h+\delta)^{4}$ where $\delta$ is a constant current necessary to obtain the correct value of the lift at $h=0$. The inner coil may be regarded as the lifting coil whereas the outer coil is the stabilising coil. Most of the losses in the plate are, therefore, supplied through the inner coil. The power input

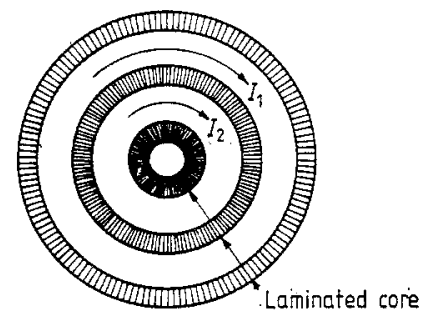

(a)

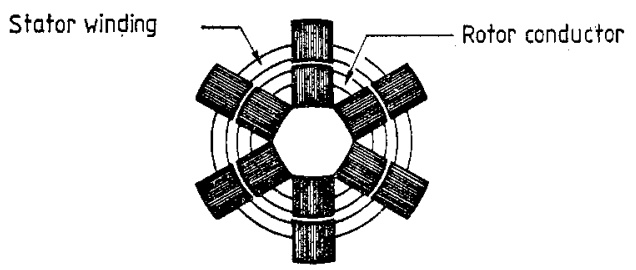

(b)

Figure 32. Configuration for disc levitators. (a) Ideal arrangement of coils and laminations, $(b)$ practical arrangement of coils and laminations. (Reproduced by permission of the IEE.) 
depends on various factors such as disc thickness, slot dimensions, etc, but for a fixed configuration such as the one for which principal dimensions are given above, the power dissipated in the disc is a linear function of the height above the stator core. Again the formula for the power loss is given (Laithwaite 1966) as $P_{\mathrm{D}}=k_{1}+k_{2} h$; the constants $k_{1}$ and $k_{2}$ can be measured in terms of the mass of the material to be lifted. For copper these are quoted as $k_{1}=27, k_{2}=36, h$ measured in $\mathrm{cm}$ and $P_{\mathbf{D}}$ in watts. For aluminium these are $k_{1}=52$ and $k_{2}=52$ for the same structure. The total power input is obtained by adding the stator $I^{2} R$ loss and the core loss to $P_{\mathrm{D}}$. The $I^{2} R$ loss increases as the fourth power of the height of levitation of the plate $h$.

5.1.1. Rectangular plates. The linear equivalent of the circular plate levitator, i.e. the one used to levitate rectangular plates, is an extension of the ideas involved in the pre-

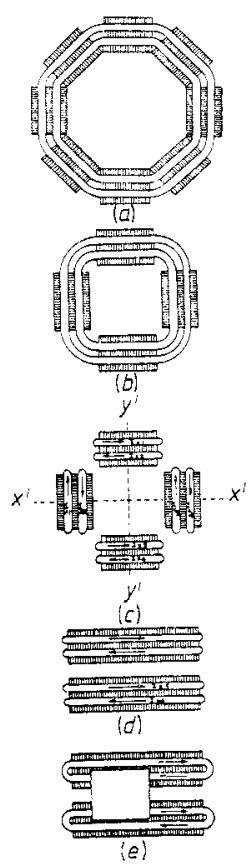

Figure 33. Evolution of a plate levitator from a disc levitator. (Reproduced by permission of the IEE.)

vious subsection. Figure 33 shows this evolution. The behaviour of the arrangement of figure $33(a)$ is very little different from that of a truly circular one. In figure $33(b)$ the number of blocks is reduced to four and a square plate of appropriate size can be supported with this arrangement. There are only two energising coils in this arrangement so that the same currents flow through the slots of the four blocks. If the currents in each slot were returned beneath the individual blocks in the manner of a Gramme ring winding (figure $33(c)$ ) currents in the eight slots could be controlled independently, thus making the stability and the attitude of the plate in each of the two directions $x-x^{\prime}$ and $y-y^{\prime}$ also independent.

The last step of this evolution is that of figure $33(d)$ in which $I_{y_{1}}=I_{y_{2}}=I_{y_{3}}=I_{y_{4}}=0$ and the blocks carrying currents along the $x$ direction have been elongated. If the system is found to be stable for $I_{x_{1}}=I_{x_{2}}=I_{x_{3}}=I_{x_{4}}$ with the directions of currents shown the arrangement of figure $33(e)$ is identical but with the advantage that the two blocks are 
now self-contained and can be moved closer or further apart to accommodate plates of different widths. Just as there is no resistance to the motion of a spinning disc, there is no resistance to the motion of the plate along the $x-x^{\prime}$ direction apart from any irregularities of construction.

5.1.2. Levitation of spheres and cylinders. Stable levitation of bodies with spherical symmetry is generally more easily obtained than with flat plates. The construction of a sphere levitator at power frequencies is basically the same as in the case of discs. It consists of two concentric coils in an iron structure and the inner coil can often be shorted out, thereby acting as a shorted ring instead of being fed from an external power source. Figure 34 shows (Laithwaite 1966) the construction of a levitator in which the hollow aluminium sphere of wall thickness $\frac{1}{32}$ in is levitated by a single coil which encloses a thick copper cylinder as a shading ring with a split iron cylinder separating the two. If thicker material, for example $\frac{3}{16}$ in wall thickness, is used for the sphere it may become

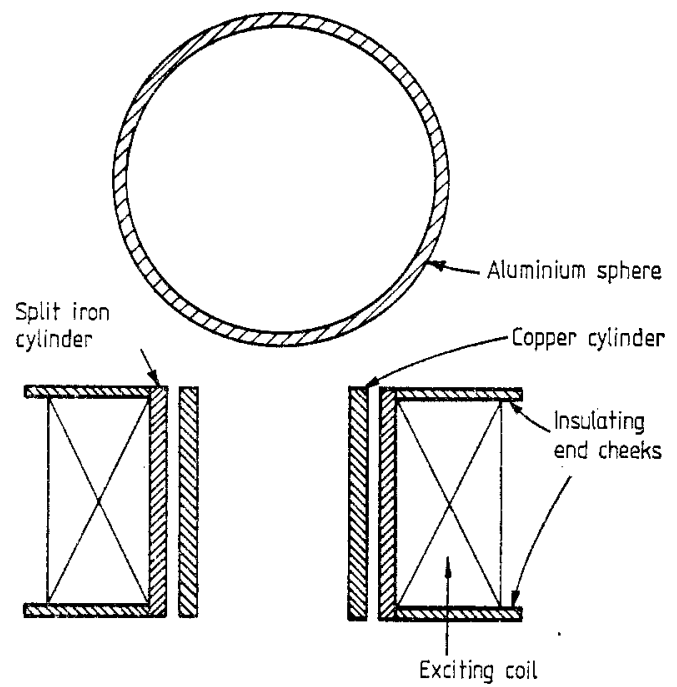

Figure 34. Levitation of a sphere using a shading ring stabiliser. (Reproduced by permission of the IEE.)

oscillatory about a horizonta] axis, finally electing to spin in one direction. The dynamic impedance of the moving conductor then so changes the flux phase pattern that the sphere drifts from the centre and is finally ejected. This occurs in such a direction that the sphere appears to roll out of the field along an invisible horizontal plane.

Support of cylinders by elongated coil systems is also possible. Spheres and cylinders behave in an analogous manner as do discs and rectangular plates as far as unresisted motion is concerned. A cylinder can be supported simply by a pair of long conductors spaced horizontally and carrying high-frequency currents in opposite directions. Again the single circular coil used to support spheres is analogous.

The techniques used in power frequency levitators are often useful in high-frequency systems. The use of iron in power frequency levitators is the same as in any conventional machine, i.e. to improve the magnetic circuits and thereby leading to a substantial reduction in $I^{2} R$ losses. It is now possible to extend the range of frequencies by the use of ferrites which have improved quite considerably. The second way in which power frequency techniques are useful to the design of high-frequency systems is in the form of 
construction. Highly rated coils or conductors such as water-cooled tubes in the same position as conductors in the slots of figure 32 can be used as an alternative to the conical coils of figure 4 which have been adopted in America. Finally the travelling field concept of power engineers is useful in deducing the best techniques for controlling the temperature of the suspended body. For a given configuration the pole pitch along one diameter, say, is fixed and increased frequencies will increase the velocity of the travelling field. This in turn will increase the power input to the supported member, thus indicating that the temperature of the levitated member and the input required to lift a given mass may be controlled by varying the frequency. The power required to lift $1 \mathrm{lb}$ of mass may be made as low as $60 \mathrm{~W}$ although it must be borne in mind that the reactive power will still be quite substantial due to an inherently poor magnetic circuit for the primary.

High-frequency levitation work is mainly concerned with supporting molten metal, and laboratory pieces of equipment to achieve this are available. These are used for zone refining and as it is achieved by a completely non-contacting method, i.e. without a crucible, it is claimed that this leads to an extremely high degree of purity. The method generally appears to be suitable (Orkress et al 1952, Polonis et al 1954, Schreibe 1953, Weisberg 1959) for application to rather small masses of non-ferrous molten metal, although Schreibe (1953) claims to have suspended $8 \mathrm{~kg}$ of molten steel. The coil arrangement of one such piece of equipment is shown in figure 4 . The conductors are copper tubes, water-cooled, and thus highly rated and the frequencies used are of the order of $10 \mathrm{kHz}$. Orkress et al (1952) have attempted an analysis of this configuration by breaking down the system into single circular loops and calculating the axial force between each loop and a solid sphere placed on the axis of the loop. It is assumed that for a sphere placed in a uniform sinusoidally time varying field, not of a high frequency, the field due to the induced eddy currents is equivalent to that of a magnetic dipole alternating in time with the field, which has a phase and amplitude depending on the strength of the generating field, the radius of the sphere, its permeability and conductivity. The net force on the sphere is zero. If the field is now non-uniform in space, the force on the sphere may be calculated by replacing it by a dipole whose moment is calculated as before in terms of a field whose properties are those of the field which exists at the centre of the sphere. Although this method is only approximate the amount of detail in the resulting formulae is considerable.

The coils shown in figure 4 consist of tubular conductors so that water may be circulated in them. With currents of the order of $800 \mathrm{~A}$ at $9.6 \mathrm{kHz}, \frac{1}{2} \mathrm{lb}$ of bronze could be supported. It is estimated that about $50 \mathrm{kV}$ A would be needed to support a few pounds of metal.

\subsection{Levitation of passenger carrying vehicles, or the magnetic river}

There are some fundamental differences between levitation of rectangular plates as described in $\S 5$ and single-sided linear motors acting against sheet secondaries with steel backing. The name, magnetic river, is intended to emphasise the behaviour of moving magnetic fields as being analogous to a viscous fluid in cases where rectangular plates levitated by one AC coil system are propelled by the same system. The moving magnetic field is regarded as a channel containing a flowing liquid into which objects can be dropped which thereafter are accelerated by the liquid and which, if unrestrained, soon attain the same speed as that of the liquid. If a piece of wood floats in the liquid stream or 'river' and is caused to pull a mechanical load on wheels along the banks of the river as shown in figure 35 some water slips past the wooden block and the latter fails to reach the river 

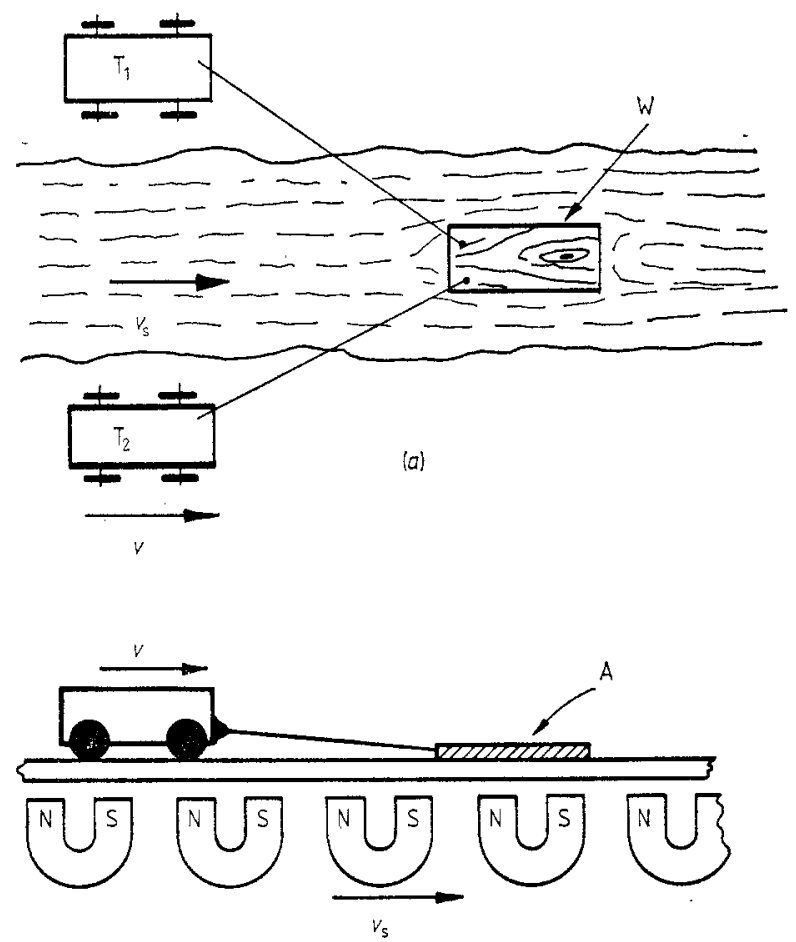

(b)

Figure 35. Magnetic river analogy of linear induction motor. Wooden block $\mathrm{W}$ in $(a)$ is replaced by a piece of conducting material $\mathrm{A}$ in (b). (Reproduced by permission of the IEE.)

speed $v_{\mathrm{s}}$. Instead, the load and the block will travel at some other speed $v$ and the entire force produced by the river on the piece of wood is transferred to the load on the banks via the connecting ropes.

There exists an analogy between the river and linear induction motors in which the secondary member is repelled as well as propelled by the primary and in the case of a machine in which the pole surface is horizontal, causes the secondary to 'float' as does the wooden block in the river. It is possible to control the lateral, vertical, pitch and roll motions of the floating secondary sheet so that it is maintained in a horizontal plane within the confines of the 'electromagnetic river'. The sheet must be made stable in yaw as well so that five degrees of freedom, viz heave, roll, pitch, yaw and lateral displacement, are under control. The sixth degree of freedom is the linear motion along the stream or the direction of the travelling magnetic field.

5.2.1. Linear induction motors as propulsion and levitation devices. It is important to look at the limitations of linear motors which may be considered as the evolution of rotary induction motors cut along the axis and rolled out flat. Machines of this kind are called axial flux machines. The two essential elements of such machines, the magnetic circuit and the electric circuit, are shown in figure 36 . When considered for application to high-speed transportation systems a long pole pitch is necessary, thereby stretching both the electric and magnetic circuit paths. This leads to large overhangs, i.e. wasted conductors on the windings and an increase in the depth of the core to carry the magnetic flux. This increases the weight of the machine and makes it almost unacceptable for a 

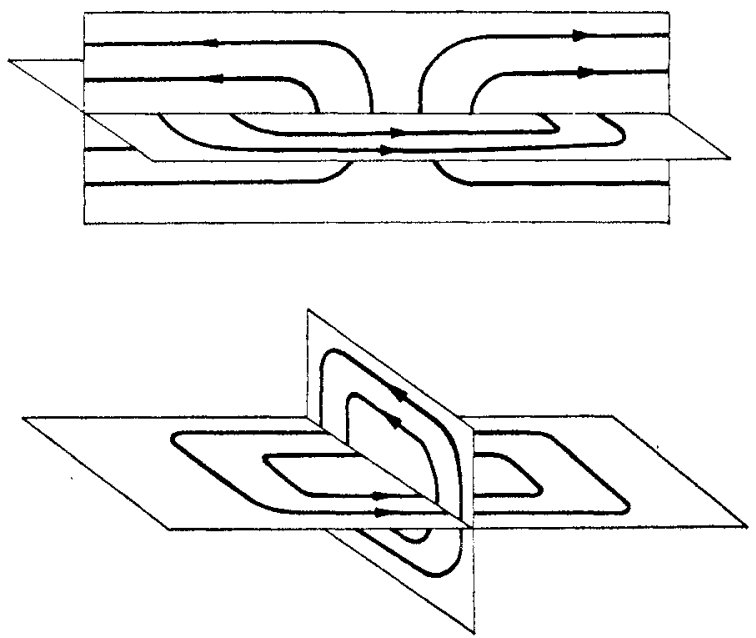

Figure 36. Electric and magnetic planes related to the direction of motion. (a) The axial flux machine, (b) the transverse flux concept. (Reproduced by permission of the IEE.)

transportation vehicle. Furthermore an axial flux motor virtually cannot be used as a single-sided machine acting against a reaction plate with backing steel (figure 37(a)) without encountering considerable forces of attraction. These forces might almost double the weight of the levitated vehicle. Such forces of attraction are also present in conventional rotary machines but if the airgap is uniform around the periphery of the rotor these forces are self-cancelling. If not these result in an unbalanced magnetic pull (UMP) and is taken up by the shaft bearings.

These fundamental objections to axial flux motors in transport applications can be overcome by using a geometry called transverse flux machines (Laithwaite et al 1971) (TFM) shown in figure 37. It can be seen from figure $37(b)$ that due to the pattern of the

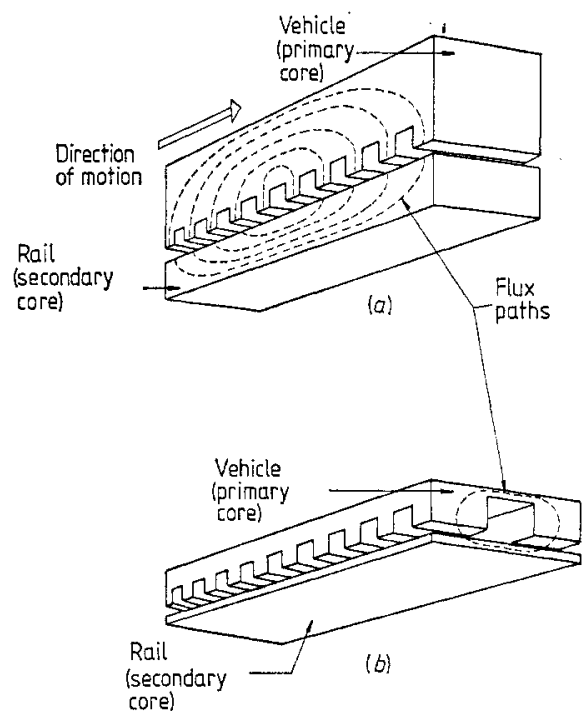

Figure 37. Comparison of flux paths, relative thickness and rails for linear motor geometries. (a) Axial flux, $(b)$ transverse flux. (Reproduced by permission of the IEE.) 
flux in the core of the machine and the reaction rail, which would normally be the track member, the thickness of the cores of both the primary and secondary would be dramatically reduced. The principal advantage of the TFM, however, becomes apparent when the equation for UMP is examined, particularly in relation to an electromagnetic arrangement where the goodness factor $G$ (Laithwaite 1966) is high and equal and opposite currents face each other across the airgap. The expression normally used for calculating the unbalanced magnetic pull is $B^{2} / 2 \mu_{0}$ and although incomplete, it is good enough in most cases of rotary machines because the force of repulsion between the opposite currents is less than $10 \%$ of the force of attraction between the magnetised surfaces. The full equation for the normal component of unbalanced magnetic pull is (Eastham and Laithwaite 1974)

$$
F_{\mathrm{n}}=\frac{B^{2}}{2 \mu_{0}}-\frac{\mu_{0} J^{2}}{2} \quad \text { per unit area. }
$$

In a typical linear motor design evolved straight from its rotary counterpart the factor $B^{2} / 2 \mu_{0}$ will still dominate. However, in a well-designed linear motor in which the slots will be wide and the teeth narrow the current density $J$ is increased and the flux density $B$ considerably reduced. Thus in equation (5.1) the two terms become much more comparable in magnitude. In fact, an increase of $J$ by a factor of three and a reduction in $B$ by a factor of five will make the second term more dominant and the force will now be of repulsion not attraction. When TFM are considered even greater lifting forces are generated although both secondary and primary members contain iron cores and this makes the design of combined lift, guidance and propulsion schemes feasible. During the course of development of TFM aimed at power factor improvement and track cost reduction it has been found that a primitive ' $\mathrm{C}$ ' core TFM would levitate and stabilise any conducting sheet of sufficient thickness and width at any height within an expanding trough as shown in figure 38. The power fed to the primary determines the height of levitation and the stability at such a height depends upon the sheet being made to fit between the dotted lines. By analogy with the unbalanced magnetic pull in rotary

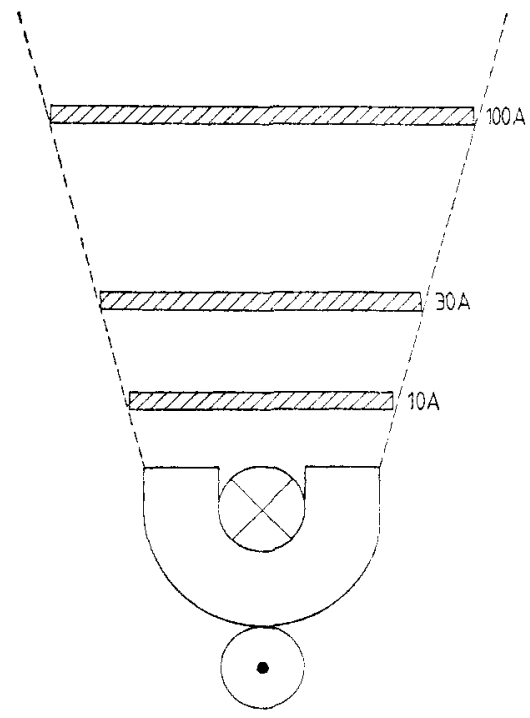

Figure 38. Expanding geometry for stable levitation. Current values are those required in the primary coil to produce the same degree of stability at the heights shown. (Reproduced by permission of the IEE.) 
machines the repulsion force available, mainly from TFM, is called unbalanced magnetic push (Freeman and Laithwaite 1968). Further the UMP is of the order of 20 times greater than the tangential or useful force and very little power, if any, is dissipated because there is no vertical motion and, therefore, no mechanical output. The levitation force is, thus, an unbalanced magnetic push exaggerated to its maximum by a single-sided arrangement and basically a force which does not require any power to levitate a mass. Whilst, of course, this argument is true it does not take account of the fact that the higher the support height, the lower will the power factor be due to secondary leakage reactance. The reactive volt ampere requirement, which is in any case substantial, will be even greater. On the other hand, it is said (Laithwaite 1977) that the bigger the machine the larger will the induced eddy current effects be when the track member consists of aluminium backed up by steel. In very large vehicles such as those for carrying 300 passengers travelling at over $300 \mathrm{mile} \mathrm{h}^{-1}$ and weighing 100-150 tons, this scheme was, therefore, claimed to be superior to the controlled DC electromagnet system. Unfortunately this has not subsequently been borne out in more detailed calculations and studies (Eastham 1978).

Early experiments showed that the normal force between the primary and secondary of a flat motor could be tensile or compressive depending upon the value of the airgap flux density $B_{\mathrm{g}}$ and the stator surface current density $J$. But as the motor gained speed lift forces were reduced and the tensile forces increased. It was also observed that the front portion of the motor would exhibit a different normal force-speed characteristic from the rear portion (Freeman and Lowther 1973) and this could give rise to pitch instability. Such instability is aggravated by increase of the goodness factor $G$ (defined as $G=p^{2} \mu \sigma \omega / \pi^{2} \rho_{\mathrm{r}} g$ ). A large airgap $g$ and a larger pole pitch $p$ in order to maintain a high value of $G$ both work in favour of the concept of a magnetic river using TFM exploiting their short magnetic circuits. A model magnetic river experimental track $9 \mathrm{~m}$ long capable of levitating aluminium plates has been demonstrated (Eastham and Balchin 1974) but it is not known whether the arrangement has subsequently been inverted as it would have to be, i.e. the wound member would be on the vehicle and the track would be an aluminium plate backed up by steel.

\subsection{The magnetic river as a vehicle system}

Eastham (1978) has calculated some theoretical boundaries of this system. He has presented two designs based on programmes developed for previous work on linear induction motors (Freeman and Lowther 1973, Eastham and Balchin 1974). One of these designs is for an airgap of $20 \mathrm{~mm}$ and the other is for a $100 \mathrm{~mm}$ airgap with a vehicle weight (assumed) of 50 tons in both cases.

\begin{tabular}{lcl}
\hline & $20 \mathrm{~mm}$ & $100 \mathrm{~mm}$ \\
\hline Motor weight & 11.2 tons & 11.2 tons \\
Synchronous capacitor weight & 19 tons & 29.5 tons \\
Corrected power factor & 1.0 & 1.0 \\
Peak efficiency & 0.38 & 0.38 \\
Weight remaining for payload & 19.8 tons & 9.3 tons \\
and body weight & & \\
\hline
\end{tabular}

The figures for operation at $100 \mathrm{~mm}$ gap, therefore, appear to leave very little margin for payload. Eastham also casts doubts about the ability to operate the vehicles at $20 \mathrm{~mm}$ 
gap without some form of feedback control and the ability to achieve the required current densities without water-cooling of conductors and excessively large area required for the motors. It is clear that whilst TFM hold considerable potential for straightforward applications as propulsion units any proposals for the use of the magnetic river require considerably more detailed studies involving the best available techniques to optimise the design.

\section{Suspension using controlled DC electromagnets}

The extent of research and development in this technology and the current activity has been indicated in \$2.8. A significant feature of the technology of controlled DC electromagnet suspension is the potential of applications to frictionless bearings and contactless suspensions. Both in vehicle applications and these energy requirements, elimination of noise and reliability appear to be important features contributing to the success of this method. Combinations of permanent magnets with controlled excitation also appear technically feasible and might lead to a further reduction of energy requirement (theoretically to zero in steady state). Being essentially a position control system the work in this area has made considerable contributions to the development of novel transducers and power amplifiers. Arising from the control of multimagnet systems it has also contributed to the advancement of theoretical work in an extremely difficult area of nonlinear multivariable control systems. At the time of writing this review controlled DC electromagnet schemes seem to hold considerable potential, as yet unexploited.

\subsection{Principle of suspension using controlled DC electromagnets}

A corollary of Earnshaw's theorem and Braunbeck's subsequent work is that systems using permanent magnets or electromagnets ( $\mathrm{AC}$ or $\mathrm{DC}$ ) without the control of current are inherently unstable. In order, therefore, to achieve stable suspension it is necessary to devise a means of regulating the current in an electromagnet using position feedback of the object to be suspended. The effect of this is to modify the force-distance characteristics such that the current and thus the force of attraction decreases as the airgap decreases and vice versa. A simple method of detecting changes in gap is the photooptical sensing method which is illustrated in figure $39(a)$. If the steel ball to be suspended in this case is attracted towards the magnet the amount of light falling on the photocell (phototransistor) diminishes which in turn decreases the current and hence the force acting against gravity. The force-distance characteristics are thus modified as shown by the broken curve in figure $39(\mathrm{c})$. In order to use this method of suspension of bodies such as vehicles the magnets and the amplifiers must be mounted on the moving member (the vehicle) and the optical 'transducer' is replaced by something more appropriate to the application. The modifications required are shown in figure $39(b)$ where the magnet is suspended below an inert steel rail and the transducer is called an inductive proximity transducer'. This is only one of the several types which may be used in this application. Since the system is a closed loop position control system some form of anticipation of position change is required in the feedback path. In the figure this is shown as compensation. The simplest form of compensation is the derivative of the position signal or phase-advance stabilisation but with more complex and multimagnet systems other forms of compensation as well as transducers are required for adequate 

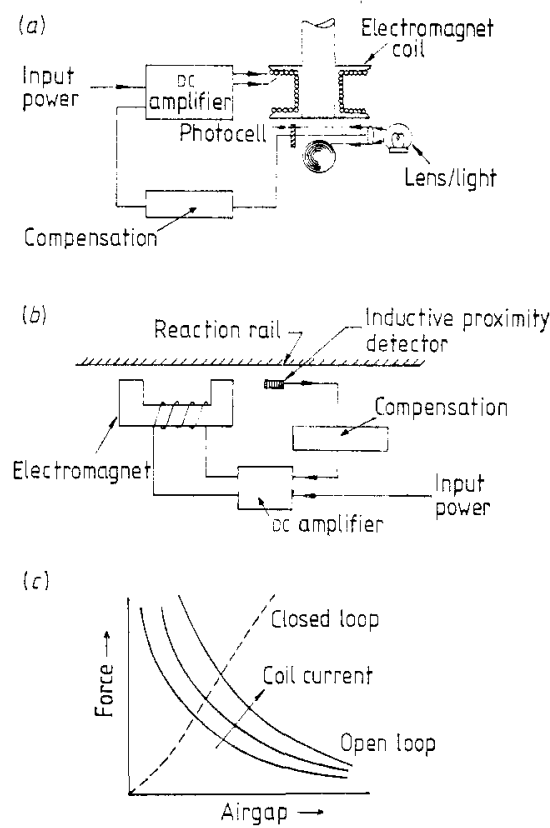

Figure 39. Principles of single-magnet suspension. (a) Suspension with optical transducer, (b) suspension of magnet under a steel rail, (c) open and closed loop characteristics.

stability. A laboratory demonstration model of a suspended steel ball is shown in figure 40 (plate).

6.1.1. Nature of the control problem in a single-magnet suspension. The force of attraction between magnetised bodies is given by

$$
F_{\mathrm{m}}=\frac{B^{2}}{2 \mu_{0}} \times \text { area. }
$$

In the case of electromagnets, such as those for suspension systems operating with an airgap, the gap flux density is directly proportional to the ampere turns $N I$ and inversely proportional to the gap length. Therefore equation (6.1) can be rewritten as

$$
F_{\mathrm{m}}=\frac{\mu_{0}}{2}\left(\frac{N I}{g}\right)^{2} \times \text { area. }
$$

It is obvious from this equation as well as the force-distance characteristics of figure $39(c)$ that the system is a highly non-linear position regulator. An adequate insight into the nature of the control problem can, however, be gained by looking at a linearised model. At the nominal required gap in figure $39(b)$ the magnet current $i_{0}$ generates a force equal to the weight to be suspended. Any displacement $z$ from this position results in a change $i$ in the current. It is assumed in the first instance that the change in the force of attraction is given by a linear function of the gap and current changes. The linearised equation is, therefore,

$$
f=-k_{1} z+k_{2} i
$$

for small changes about the equilibrium position where $k_{1}$ is the force per metre at constant current and $k_{2}$ is the force per ampere at constant distance. The constants $k_{1}$ and 
$k_{2}$ can be determined experimentally for a given magnet. Thus

$$
m \ddot{z}=-f=-\left(-k_{1} z+k_{2} i\right)
$$

where $m$ is the mass of the suspended body.

The relationship between the magnet current and voltage is

$$
R i+L \frac{\mathrm{d} i}{\mathrm{~d} t}=V .
$$

In Laplace transform notation equations (6.4) and (6.5) become

$$
\left(s^{2}-k_{1} / m\right) Z(s)=\frac{k_{2}}{m} I(s)
$$

and

$$
I(s)=\frac{k}{1+s T_{\mathrm{m}}} V(s)
$$

The two equations combined together give

$$
\frac{Z}{V}(s)=\frac{k_{1} k_{2}}{m\left(1+s T_{\mathrm{m}}\right)\left(s^{2}-k_{1} / m\right)} .
$$

The quantity that can be directly controlled through a DC amplifier driving the magnet is its voltage output and $T_{\mathrm{m}}$ represents the lag in the resulting current due largely to the magnet inductance. The second term $\left(s^{2}-k_{1} / m\right)$, however, arises from the force-distance characteristics which may be considered analogous to a spring stiffness constant but in this case a negative one. The consequence of the negative spring stiffness characterised by $k_{1}$ causes these systems to be inherently unstable. The explanation in control terminology is that the term $\left(s^{2}-k_{1} / m\right)$ represents poles at $\pm\left(k_{1} / m\right)^{1 / 2}$ on the $s$ plane and the pole at $+\left(k_{1} / m\right)^{1 / 2}$ contributes to the instability. In the closed loop or feedback systems of figure 39 the transducers will have a very small time constant associated with it but it is of very little practical significance here in comparison with the main time constants. Because the compensation is in series with the transducer or the actuating signal and the amplifier driving the magnet this scheme is known as series or cascade compensation. A block schematic of the system is shown in figure 41 .

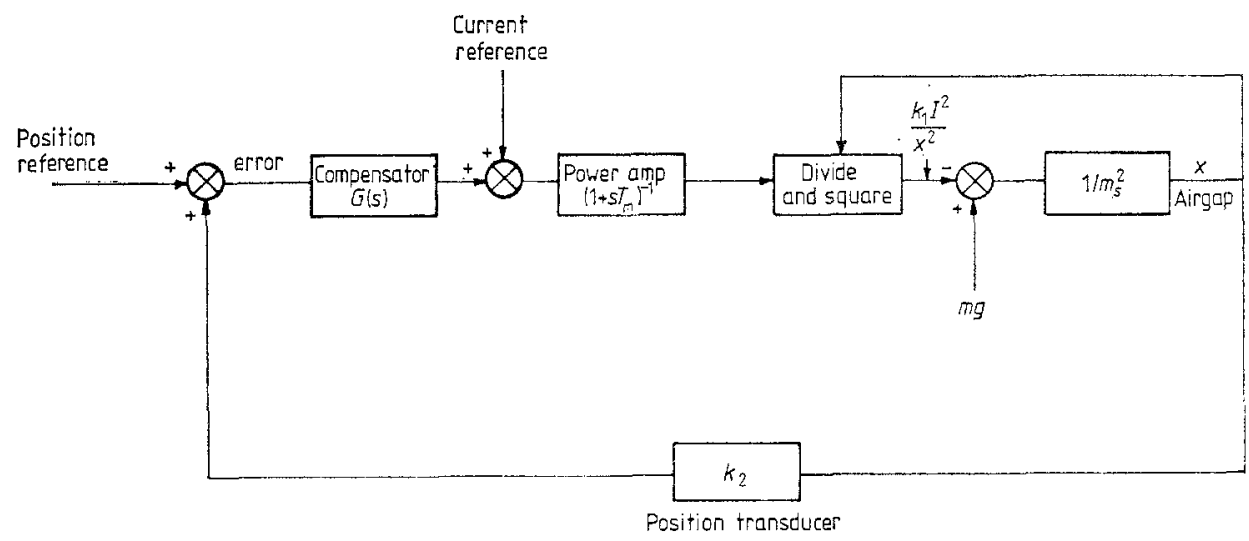

Figure 41. Schematic of a general magnetic suspension with cascade compensation. 
6.1.2. Multimagnet systems. In multimagnet systems and particularly in transport applications there are some departures from the single-magnet systems. By and large, when providing maximum lift the magnet is operating in a highly non-linear part of its force-distance characteristics. The control system gain is directly related to the slope of the characteristics at the operating point. Therefore changes in the operating conditions such as load variations will severely degrade the transient response or even cause instability. Another important departure of the multimagnet systems arises from the mechanical coupling of magnets mounted on a rigid chassis and that of the individual control systems of the magnets. This demands an even higher degree of stability than that obtainable from the cascade compensation scheme using position transducers alone.

There are several ways of overcoming or at least reducing the severity of the problem of mechanical interaction, some of which are described in \$6.2.1. There is, however, some case for further improvements through the basic control system design. If a transducer is introduced on the face of the lift magnets to measure gap flux density and used in conjunction with a feedback control loop to control the magnet current in such a manner that the gap flux density remains constant over the operating range of airgaps, the non-linear force-distance characteristics will be very nearly linear. A primary cause of instability will thus be eliminated, at least in the operating region, by the use of a separate flux control loop. Although other transducers have variously been used the author has used a Hall effect device mounted on the face of the magnets for the simple reason that the output from a Hall plate is DC. The use of a flux control loop means that the force is now independent of the gap length or that in equation (6.3) the constant $k_{1}$ is zero. Alternatively this also means that in the $s$ plane the pole in the right-hand half at $+\left(k_{1} / m\right)^{1 / 2}$ will have effectively moved to the origin. This partial linearisation leaving the force variations proportional to $i^{2}$ gives a dramatic improvement to the stability margin of the multimagnet systems. Introduction of this loop to keep the airgap density constant (for varying $z$ ) changes the open loop system equation (6.7) to

$$
\frac{Z}{V}(s)=\frac{k_{1} k_{2}}{m\left(1+s T_{\mathrm{m}}\right) s^{2}}
$$

The scheme of using an independent feedback loop for one parameter, gap flux density in this case, can be extended to the measurement of position and velocity independently and to using a separate loop for each. This concept of partial state feedback gives control over gains of the individual loops and enables optimum setting for each. The transducers employed for measurement and position are described later but the measurement of acceleration and integrating the accelerometer signal to give velocity has the advantage that the noise is attenuated in the process of integration. Obtaining velocity from the position signal by taking its derivative or by differentiating it, on the other hand, introduces a great deal of noise. In particular, if the position signal is obtained by a process of rectification of an AC voltage the problem can be really acute. A block schematic of a single-magnet system using separate position, velocity and flux transducers is shown in figure 42 .

An efficient suspension system employing DC electromagnets must of necessity operate at relatively small airgaps as compared to, say, the superconducting levitation system. The control of the airgap must, therefore, be very tight and for passenger carrying vehicles this introduces conflicting requirements. Tight control of airgaps or a stiff suspension will produce a hard and uncomfortable ride. It is, therefore, commonly accepted that acceleration feedback is also necessary to achieve ride comfort. 


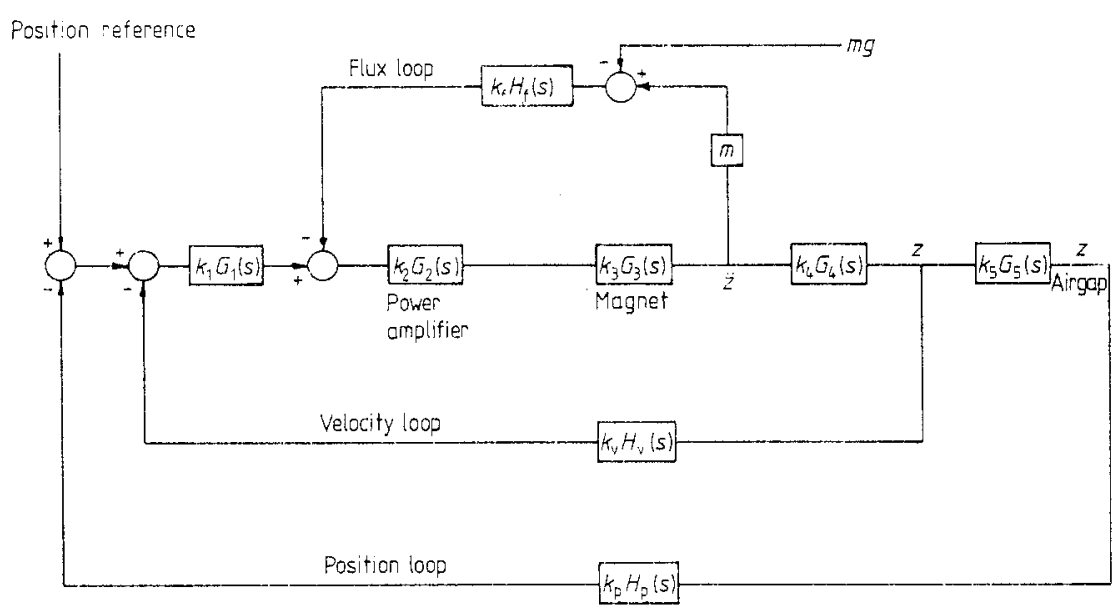

Figure 42. Schematic of magnetic suspension with partial state feedback controller.

\subsection{Analytical aspects of multimagnet systems}

The problems of stabilising and extending the analysis of single-magnet systems to multimagnet systems are many and formidable. These are attributable in the main to the inherent non-linearities in the control systems and to the mechanical cross coupling between the magnets and their controllers all mounted on the same framework. For example, the attitude of a vehicle chassis in free space is determined by three parameters: heave, roll and pitch. If the chassis has four magnets, one at each corner, by specifying and attempting to control four gaps to be the same introduces conflict between control loops for each corner. This may be called 'local control'. Alternatively, the measurement of the airgaps at the four corners may be converted to give the attitude of the chassis or the suspended body in terms of heave, roll and pitch and these are controlled instead by associating a reference as well as compensation with each of them. This may be termed 'integrated control'. The concepts of 'local' and 'integrated' control are illustrated in figure 43. This figure illustrates the problem which is that multimagnet frames are non-linear multivariable control systems and that the objectives are to achieve decoupling by whatever means are practicable. These and various other aspects of the control of magnetically suspended vehicles, such as the operation on flexible guideways, have received a great deal of attention within the last decade (Linder 1976, Jayawant et al 1975, 1976, Jayawant and Sinha 1977, Gottzein and Lange 1975, Gottzein et al 1975, 1977, 1979, Nakamura 1979, Nakamura et al 1979, Katz et al 1974, Popp and Schiehlen 1975, Meisinger 1975, 1977, Sinha 1977a, b). This work on vehicle dynamics is applicable with only minor modifications to vehicles using any other method of levitation such as superconducting magnetic levitation. Only a brief introductory outline of it is given here.

6.2.1. Equations of motion and geometric transformations. A free body in space has six degrees of freedom, three associated with translational motion, the other three with rotation. Given information about all external forces acting on the body the principles of linear and angular momentum lead to six non-linear differential equations. These contain common factors giving rise to interaction between the variables.

Consider the suspended body to be represented by a uniform box-shaped object with the centre of mass coincident with the centre of geometry (figure 44) and the frames of 

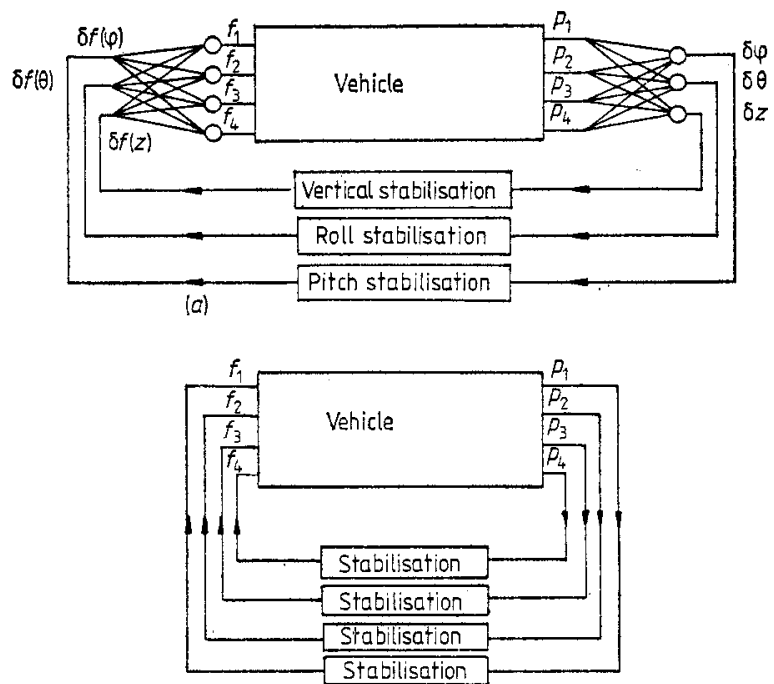

(b)

Figure 43. Control strategies for multimagnet systems. (a) Separate control of each degree of freedom, (b) control of each magnet-transducer pair.

reference and the principal axes as shown. The principle of linear momentum gives the following equations (Hazlerigg 1974):

$$
m \ddot{x}=F x \quad m \ddot{y}=F y \quad m \ddot{z}=F z
$$

where $F x, F y$ and $F z$ are the resultant forces acting along the inertial axes $\mathrm{O} x, \mathrm{O} y$ and $\mathrm{O} z$.

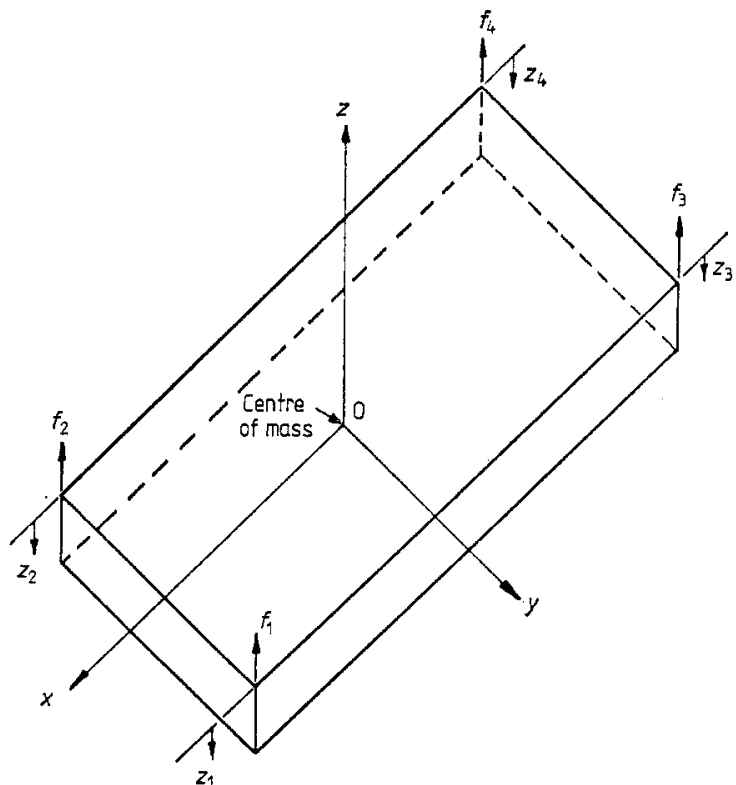

Figure 44. Frame of reference and dimensions of quantities used in the equations of dynamics. $\mathrm{O} x$, propulsion axis; $\mathrm{O} y$, lateral guidance axis; $\mathrm{O} z$, heave axis; $B$, distance between centres of lift along $y$ axis; $b$, distance between position transducers along $y$ axis; $D$, diagonal distance between centres of lift; $d$, diagonal distance between position transducers; $L$, distance between centres of lift along $x$ axis; $l$, distance between position transducers along $x$ axis. 
The principle of angular momentum leads to three more equations for the rotational coordinates. From Euler's equations after substitution of appropriate Euler's angles

$$
\begin{aligned}
& T_{x}=I_{x x} \dot{\omega}_{x}+\left(I_{z z}-I_{y y}\right) \omega_{y} \omega_{z} \\
& T_{y}=I_{y y} \dot{\omega}_{y}+\left(I_{x x}-I_{z z}\right) \omega_{z} \omega_{x} \\
& T_{z}=I_{z z} \dot{\omega}_{z}+\left(I_{y y}-I_{x x}\right) \omega_{x} \omega_{y} .
\end{aligned}
$$

A suitable set of Euler's angles is shown in figure 44 which corresponds to the notion of rolling, pitching and yawing of the vehicle frame. In terms of these angles the angular velocities are

$$
\begin{aligned}
& \omega_{x}=\dot{\varphi}+\dot{\psi} \cos \theta \\
& \omega_{y}=\dot{\theta} \sin \varphi-\dot{\psi} \sin \theta \cos \varphi \\
& \omega_{z}=\dot{\theta} \cos \varphi+\dot{\psi} \sin \theta \sin \varphi
\end{aligned}
$$

and

$$
\begin{aligned}
& \dot{\omega}_{x}=\ddot{\varphi}+(\ddot{\psi} \theta+\dot{\psi} \dot{\theta}) \\
& \dot{\omega}_{y}=\ddot{\theta}+(\dot{\psi} \dot{\theta} \varphi \theta-\ddot{\psi} \varphi-\dot{\psi} \dot{\varphi}-\dot{\theta} \dot{\varphi} \varphi) \\
& \dot{\omega}_{z}=\ddot{\psi}+(\ddot{\theta} \varphi+\dot{\theta} \dot{\varphi}-\dot{\psi} \dot{\varphi} \varphi-\dot{\psi} \dot{\theta} \theta) .
\end{aligned}
$$

For small angular motions the terms in brackets may be neglected on the grounds of smallness as compared to the principal terms $\ddot{\varphi}, \ddot{\theta}$ and $\ddot{\psi}$, i.e. in comparison with the roll, pitch and yaw accelerations. The resulting equations for angular motions and torques become

$$
T_{x}=I_{x x} \ddot{\varphi}(\text { roll }) \quad T_{y}=I_{y y} \ddot{\theta}(\text { pitch }) \quad T_{z}=I_{z z} \ddot{\psi} \text { (yaw). (6.11(a)) }
$$

$T_{x}, T_{y}$ and $T_{z}$ are the applied moments of the external magnet forces. In practice, the platform or the suspended frame may not be absolutely rigid and hence one more equation describing the torsional motion of the suspended platform may be necessary and a linearised version of this is

$$
T_{t}=I_{t} \ddot{\zeta}
$$

In practice the forces and torques in equations (6.9) and (6.11) are derived from a system of electromagnets and the displacements and rotations are measured by a system of transducers. Since the forces, torques, displacements and rotations are not measured directly in the form in which they occur in equation (6.9)-(6.11) the magnets and transducer systems effectively introduce cross coupling. The four lift forces generated by the four corner magnets $f_{1}, f_{2}, f_{3}, f_{4}$ are controlled by outputs $p_{1}, p_{2}, p_{3}, p_{4}$ from a set of four transducers so as to maintain roll and pitch angles at zero and the height (of the vehicle) constant. Then in figure 45

$$
\begin{gathered}
F_{z}=\frac{1}{4}\left(f_{1}+f_{2}+f_{3}+f_{4}\right) \\
T_{x}=\left(-f_{1}+f_{2}-f_{3}+f_{4}\right) B \\
T_{y}=\left(f_{1}+f_{2}-f_{3}-f_{4}\right) L \\
z=\frac{1}{4}\left(z_{1}+z_{2}+z_{3}+z_{4}\right) \quad \varphi=\frac{1}{2 b} \sum_{i=1}^{4} \pm z_{i} \quad \theta=\frac{1}{2 l} \sum_{i=1}^{4} \pm z_{i} \quad \zeta=\frac{1}{2 d} \sum_{i=1}^{4} \pm z_{i} .
\end{gathered}
$$

Thus the platform (vehicle) can be regarded as a transformation between the magnet forces and the transducer outputs and is in effect a four-input, four-output system. 


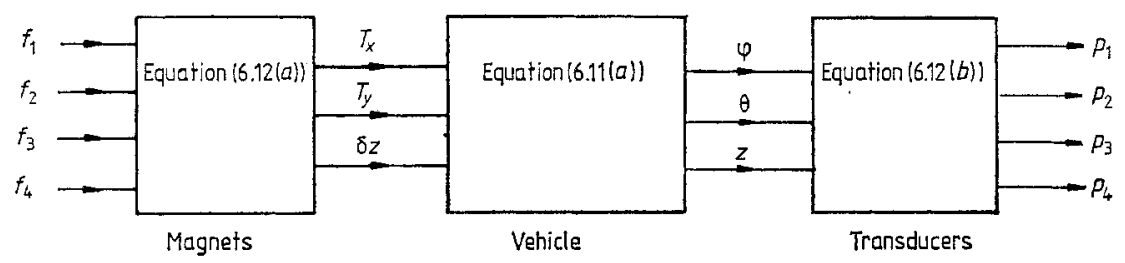

Figure 45. Vehicle dynamics.

Change in any one of the forces will cause a response in all the output variables in view of the cross coupling implied by equations (6.12). However, the essentially non-interacting dynamics represented by equations $(6.11)$ enables in principle each degree of freedom $\varphi, \theta$ and $z$ to be controlled independently by working with the appropriate linear combination of the $F_{i}$ and $p_{i}$ (figure 43(a)). An alternative design procedure is to admit the cross coupling implied in equations (6.12) and to attempt control based on a tight association between a magnet and its nearest transducer, i.e. the force $f_{i}$ is controlled by the transducer $p_{i}$. This leads to the control loop shown in figure $43(b)$. In this case the disturbance entering any loop or applied to the mechanics in the form of external forces will cause changes to all variables and will propagate throughout the system. It is assumed in the analysis that all magnet-transducer pairs are identical, a state of affairs seldom likely to be achieved in practice. There will, therefore, always be interactions both static and dynamic in multimagnet vehicles. Furthermore, although complete static non-interaction may just possibly be achieved, dynamic non-interaction under all operating conditions is a virtual impossibility due to higher-order effects and also due to the centre of mass being differently placed from the centre of geometry. This will, therefore, introduce coupling between various modes. In the realms of control theory it is also possible to achieve decoupling by various state feedback decoupling methods. As yet there are no reports of any group of workers having achieved any success through these.

6.2.2. Decoupling through flexible chassis or magnet mountings. A somewhat simpler alternative to schemes of dynamic decoupling by the use of state feedback methods is to introduce flexibility in the vehicle chassis. The advantage of this method is that it does not require any electronic circuitry other than that for individual magnet stabilisation. This scheme was implemented by the author in one of the vehicles (figure 46). In this vehicle

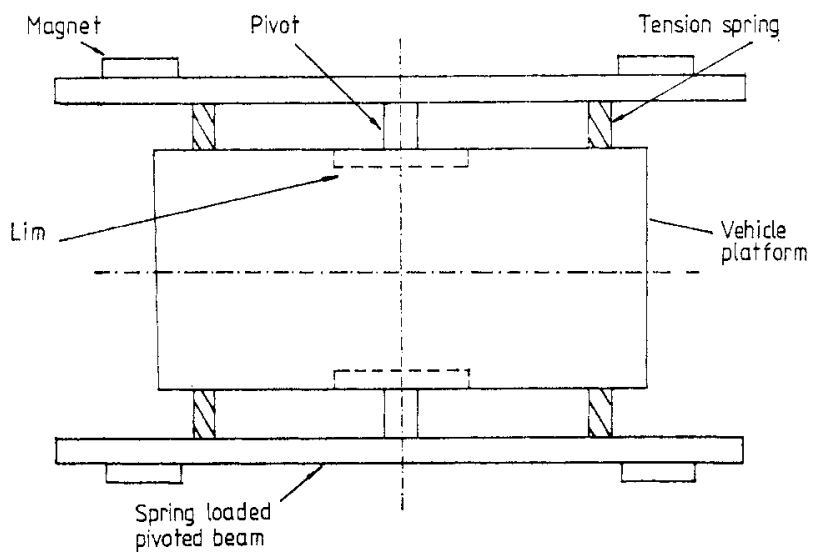

Figure 46. Flexible suspension frame. 
platform the magnets are mounted as a pair on beams which are pivoted about their centres and coupled to the vehicle platform by means of springs of appropriate stiffness. There is enormous improvement in the overall performance and stability of this arrangement. The same principle of a sprung chassis was incorporated in the General Motors vehicle and in the Japan Air Lines vehicle HSST 02.

The concept of independent control of magnets and sprung chassis has been carried even further on the German vehicles, Transrapid 04 and Transrapid 05 (figure 7). In these vehicles the magnets are attached via a primary suspension to magnet frames which are connected to the cabin by a secondary suspension. This structure adapts to guideway irregularities even at high speeds and therefore a smaller airgap may be realised, leading to a considerable reduction in magnet weight and power required for suspension. This modularised structure carrying up to four magnets per frame has been termed the 'magnet wheel' (Gottzein and Crämer 1977).

\subsection{Transducers, magnets and power amplifiers for magnetic suspension systems}

The principal elements of a suspension system using controlled DC electromagnets, being a part of a control system, are subject to criteria of dynamic performance in much the same way as are components of any electromechanical control system. When the suspension system forms part of the vehicle-mounted equipment it is then subject to a multitude of other criteria such as weight, power consumption, reliability and ruggedness as well as safety requirements. Many of these requirements are mutually conflicting and the process of optimisation is difficult. The amount of effort devoted to the development of vehicles in the last decade has, however, been such that remarkable progress has been made in the component field and a review of some of these is likely to prove useful. Moreover the application of magnetic suspension to fields other than vehicle transportation is at least as important.

6.3.1. Transducers. In magnetic suspension systems transducers are required for the measurement of position velocity and acceleration. In addition the flux control loop described in $\S 6.1 .2$ also requires a transducer to measure the gap flux density. Whilst a wide variety of transducers appear to have been advocated only two distinct types now seem to be in favour. All transducers need to be judged by some criteria and the following are some of the important ones. In setting out these criteria it is worth noting that in a control system the transducers operate outside the feedback loop. The accuracy of the transducers cannot be improved by any compensation techniques and the control system is at best only as good as the transducers in it.

(i) Bandwidth: this may be as large as $1000 \mathrm{rad} \mathrm{s}^{-1}$.

(ii) Robustness and stability under various operating conditions.

(iii) Linearity over the operating range.

(iv) Immunity from external noise, radiation and strong magnetic fields.

(v) Ability to operate without mechanical contact.

6.3.1.1. Optical. The simplest type of position transducer that can be used is the one illustrated in figure 39. Either a filament type of light source or an infrared source can be used. With this transducer the output is linear over a $2 \mathrm{~mm}$ range of movement and the bandwidth is as high as $10 \mathrm{MHz}$. Very high stiffnesses of suspension and precision can be achieved by using optical transducers but the application is limited to a clean environment only. 
6.3.1.2. Inductive. The basic principle employed in inductive transducers is that the inductance of an iron- or air-cored coil is dependent on its proximity to a ferromagnetic body or more particularly on the airgap between the two (Barwick et al 1977). The transducer measuring element is usually an E-shaped or U-shaped ferrite or high permeability steel laminated core (such as $\mu$-metal) with several hundred turns on it. A widely used form of inductive transducer is constructed using the identical coils (Hazlerigg 1974), one being the transducer and the other a reference coil (figure 47). The coils are elements of a Maxwell bridge which is in balance when the inductance of the two coils is equal. Any imbalance of the bridge due to airgap variations of the transducer coil is fed to a phase-sensitive detector to discriminate the direction of displacement. The bandwidth is limited by the filter circuitry but the transducer can be made to give linear output over a large range. A variant of the inductive transducer as described above is one which operates against a non-magnetic conducting surface such as an aluminium sheet. The

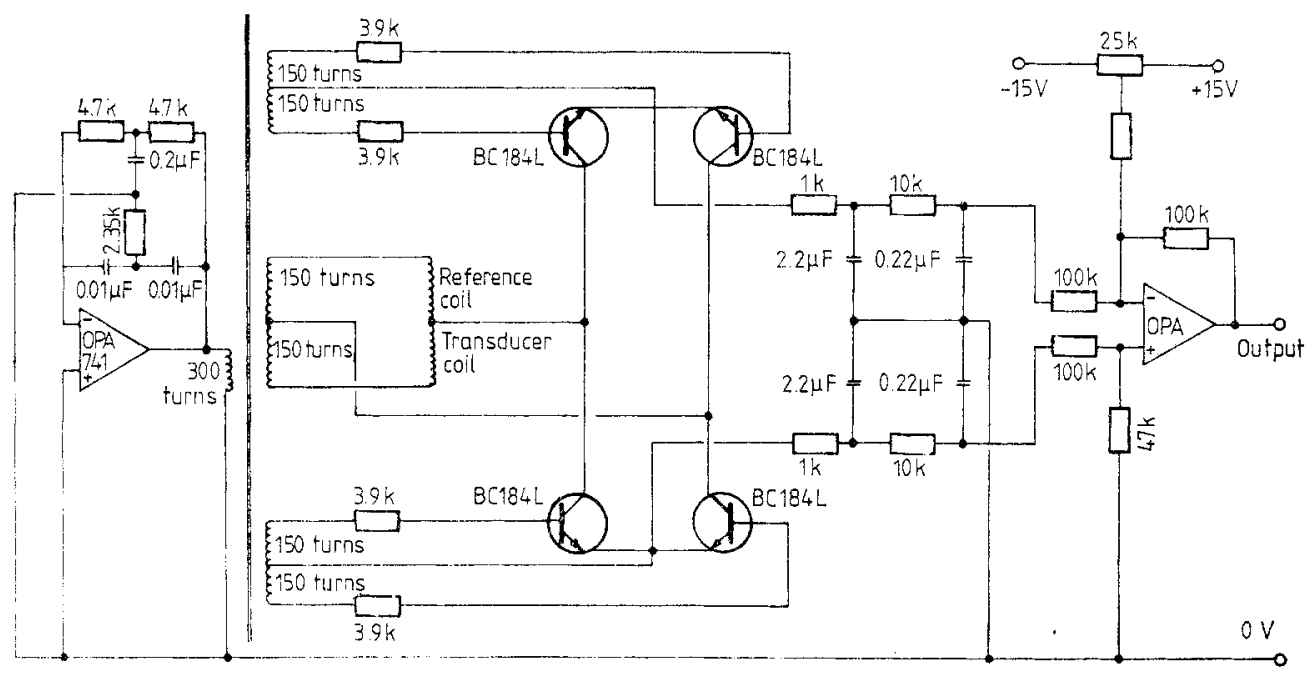

Figure 47. Circuit for inductive position transducer with bridge and phase-sensitive rectifier.

change in inductance of the coil in this case is due to the induced eddy currents (Sinha 1977b).

6.3.1.3. Magnetic. A magnetic transducer consists of a permanent magnet with a Hall plate on its face measuring the gap flux density when placed in the proximity of another ferromagnetic surface. The closer the magnet is to the rail, the greater the flux density. This is partly due to the change in the operating point on the demagnetisation curve of the magnet and partly due to the leakage flux being higher at larger gaps. A circuit developed by Hodkinson (1972) takes the Hall voltage $V_{\mathrm{H}}$ and uses it to control the Hall current $I_{\mathrm{HI}}$ such that $V_{\mathrm{H}}$ remains constant. In effect, the flux is multiplied by a current proportional to the output voltage to achieve linearisation. The circuit is shown in figure 48. It has been found (Jayawant et al 1975) that for small gaps where the flux density $B$ approximately varies as the inverse of the gap, i.e. as $1 / z$, and also a fraction $\alpha$ of the output of the amplifier $A_{2}$ is fed back to its positive input terminals, the output is given by

$$
V_{0}=\frac{V_{\text {ref }}}{B / R-\alpha}=\frac{c_{1} z}{c_{2}-c_{3} z} .
$$




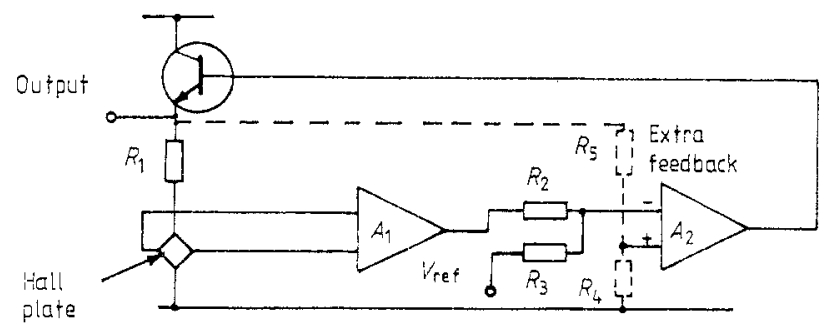

Figure 48. Hall plate position transducer.

By an appropriate selection of the constants $c_{2}$ and $c_{3}$ a virtually linear output can be obtained for a range of $z<c_{2} / c_{3}$. The bandwidth of these transducers is in excess of a few $\mathrm{kHz}$. The principal advantages of this transducer are that the output is $\mathrm{DC}$, it is virtually immune from noise and is of a fairly rugged construction.

These transducers are preferably used in conjuction with separate velocity and flux control loops as shown in figure 42. In the small instrument type of suspension, however, where separate transducers might be precluded on account of space restrictions, a modification shown in figure 49 has been found suitable instead. For a particular geometry, the gap flux density $B=k N I / z$. Hence the gap can be determined from the quantity $I / B$. In figure 49 the Hall plate is placed in the feedback path of the amplifier, thus acting as a divider. When the amplification factor of the amplifier is large and since $I_{\mathrm{H}}$ is now determined by both $B$ and $V_{0}$, it is easy to show that

$$
\begin{aligned}
V_{0} & =\frac{R}{k_{1}{ }^{\prime} B}\left(k_{2} I_{\mathrm{m}}+k_{3}\right) \\
& =k_{\mathrm{A}} \frac{\left(I_{\mathrm{m}}+k_{\mathrm{B}}\right)}{B}
\end{aligned}
$$

when $k_{\mathrm{B}}=0$ the output voltage $V_{0} \propto I / B$ or $z$. This transducer has been called the $I / B$ transducer (Whorlow 1978).

6.3.2. Magnets. The design of magnets for providing lift in magnetic suspension is constrained by static design considerations on the one hand but also by dynamic considerations. Given that the magnets are to operate with airgaps of the order of $5-15 \mathrm{~mm}$ the object of the design is to maximise the lift per unit power input and if the magnets are intended for vehicle applications then to also minimise the weight of the magnet per unit

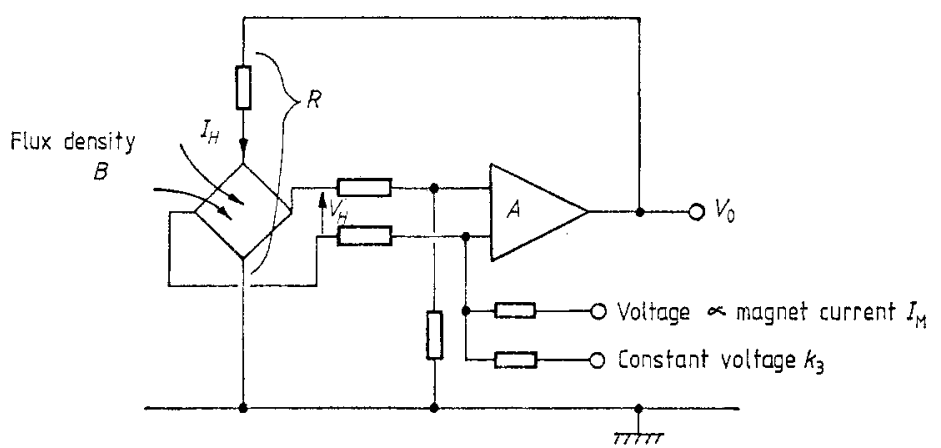

Figure 49. $I / B$ transducer. 
lift capability. Amongst the considerations which limit the static performance of lift magnets are that the magnet material is ordinary mild steel, thus placing a relatively low limit of flux density for saturation, and that the width of the track or the lift rail is limited, thus restricting the window area and the pole face area.

The starting point of the design is to consider equation (6.2) giving the force of attraction:

$$
F \propto \frac{(N I)^{2} \mu_{0}}{z^{2}} A
$$

where $N I=$ ampere turns, $z=$ airgap, $A=$ pole face area.

The constant of proportionality is determined by the track and magnet geometry. Over and above the useful flux which links both the magnet and the rail there is a considerable amount of leakage flux. It is therefore counterproductive to make the magnets, if they are U-shaped, very much deeper than they are wide. In a given window area the choice of coil materials rests between either copper or anodised aluminium. The latter gives coils with much improved heat-dissipating capability but results in low-voltage high-current coils. This is no particular disadvantage since it can be shown (Gondhalekar 1980) that on the basis of the forcing voltage to quiescent voltage ratio a high-current design is to be preferred although a given window area produces the same time constant. The commonly accepted choice of geometry for magnets is $\mathrm{U}$-shaped, thus giving long magnets travelling along this length. Magnets travelling at right angles and presenting the track with alternating N-S polarities suffer from excessive induced eddy currents. These result in increased drag forces, attenuation of the airgap flux density and thus serious loss of lift at speed without a laminated track.

The factors discussed above also influence the dynamic characteristics and the following features need particular consideration: $(a)$ inductance of coils and time constant, (b) operating voltage and voltage needed to force currents to change rapidly enough, $(c)$ lateral guidance forces, $(d)$ induced eddy current effects and $(e)$ route switching of vehicles.

The time constants of magnets for 1-2 ton lift capability are of the order of several hundred milliseconds and in order to obtain bandwidths of $10-25 \mathrm{~Hz}$ a forcing voltage of about 3-5 times the steady-state voltage drop may be required. It is then possible to calculate the rate at which the generated force can change (Jayawant 1981), i.e. the slewing rate of the force.

The guidance forces generated by lateral displacement of magnets as shown in figure 50 are unlikely to be of sufficient magnitude for vehicle applications, even if currents are increased to compensate for the reduction in the pole area to keep the airgap (and lift) constant. By displacing magnets first on one side of the rail as shown in figure 50 and then on the other and using twice the number of magnets to those required it has been demonstrated on the German vehicle Transrapid 01 that sufficient guidance forces can be generated (the US Department of Transportation figure is $42 \%$ of the lift force). For higher speed vehicles, capable of travelling in excess of $400 \mathrm{~km} \mathrm{~h}^{-1}$, it appears that separate guidance magnets are preferred (Gottzein et al 1977) on the grounds of avoiding interaction between control systems of the guidance and lift functions.

The induced eddy currents in the rails due to the relative motion between the magnets and the rails has a two-fold effect. In the same manner as in the case of superconducting levitation it will produce an extra drag with similar speed-dependent characteristics. A much more serious effect is, however, that the eddy currents will tend to reduce the airgap flux density quite significantly and since lift is proportional to the square of the flux 


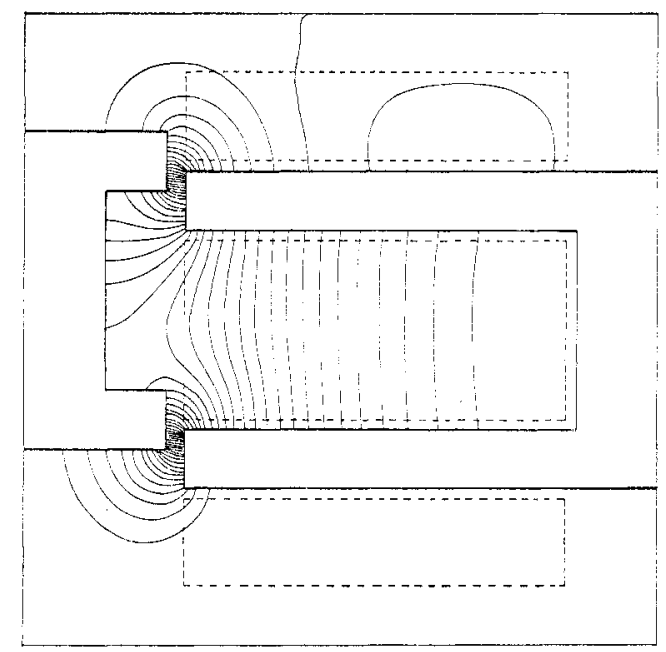

Figure 50. Computed field plot for a U-shaped magnet in a laterally displaced position.

density this will result in a drastic reduction in the lift capability of the magnets. There appears to be little published literature (Yamamura and Ito 1975) on the subject, partly in the belief that laminated rails will have to be used for high-speed vehicles and partly because the problem is a genuinely difficult one to compute or evaluate experimentally. An alternative solution to that of laminated rails appears to be through the use of 'magnet wheels', i.e. a number of short-length magnets as closely spaced as possible operating at small airgaps. Results of this approach (Crämer 1979) seem to indicate that the rail stays magnetised as the short magnets pass over a given section of the rail at high speeds and particularly at small gaps the induced eddy currents are reduced to a level not large enough to cause any concern at speeds of up to $500 \mathrm{~km} \mathrm{~h}^{-1}$.

The problem of changing routes, switching as it is called, for magnetically suspended vehicles is rather a difficult one. In the Emsland project it is proposed to shift sections of the track in much the same way as is done for railways. Proposals were made (Barwick et al 1977, Domande 1973) for using duplicate sets of magnets and duplicated rails at switches but do not appear to have provided any experimental evidence. A small $\frac{1}{4}$ ton vehicle using a new geometry (Jayawant 1977, 1979) called the I magnet has been tested by this author and is illustrated in figure 51 (plate). The advantage of this scheme is that the vehicle will operate with an inverted L-shaped rail either on one side of the magnets or the other or both. Hence there is no need to turn the magnets on and off as required in the duplicate magnet scheme.

6.3.3. Power amplifiers. The recent rapid advances in the technology of attraction magnetic suspension are attributable almost entirely to the development of solid-state electronic devices and power devices at that. High power to weight ratio and efficiency coupled with extreme reliability of operation are principal requirements for magnetic suspension in almost any application. The early experimenters (Kemper 1937) had to use valve amplifiers and although they succeeded in suspending substantial loads it is not surprising that this work did not lead to many (if any) practical applications at the time.

As pointed out in the previous subsection the time constants of typical magnets are of the order of several hundred milliseconds and yet the magnet-amplifier combination 
must act as a closed loop control system of a bandwidth of at least $10 \mathrm{~Hz}$ or more. Fairly substantial reserve voltage to force rapid current changes in the magnet in order to overcome the inductive voltage is, therefore, an essential feature in the design of DC power amplifiers in this context. This requirement in turn can lead to large power dissipation and low efficiencies in quiescent operating conditions.

The choice of amplifier configuration is essentially between Class A and Class D. These two configurations are illustrated in figure 52. The active linear regulator or the active switch is either a transistor or a thyristor. The Class A amplifier is obviously preferable, because of its smooth current regulation capability, to Class D where the on-off sequence is bound to interfere with the transducers, particularly the flux loop transducers which are mounted on the magnet faces. However, when the efficiencies of the two schemes are considered in the light of power dissipated in the output stage (transistors) it is quite clear that for large power applications Class D is the only real choice (Hodkinson 1974). There are several problems associated with the use of Class D amplifiers such as the added complexity of the pulse-width modulators, although this offers the possibility of isolation between control and power circuits, using either transformers or opto-isolators. There are often problems of both audio noise as well as RF interference with other circuits, but these are not serious.

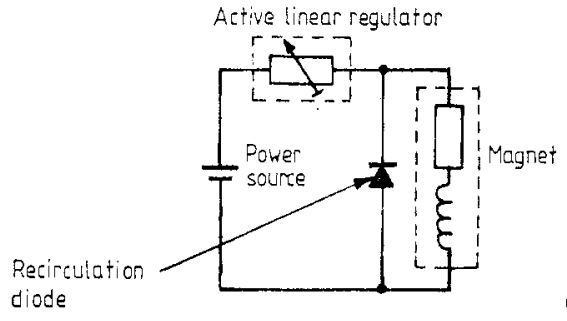

$\{a\}$

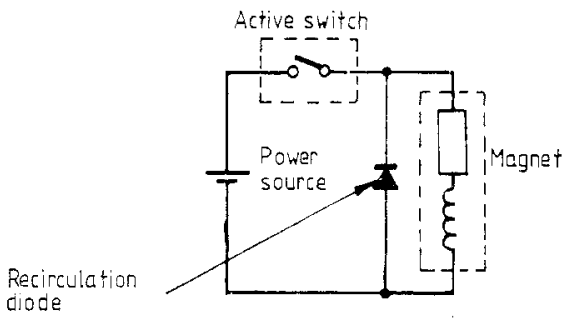

(b)

Figure 52. Types of power amplifiers. (a) Class A, (b) class D.

\subsection{Contactless support and frictionless bearing applications of controlled DC electro- magnetic suspension}

The current popularity of suspension and levitation stems no doubt from the possibilities in high-speed ground transportation schemes. Whilst these are both challenging and exciting there is considerable scope for the application of suspension techniques to achieving frictionless bearings. The requirement in this case is often for close tolerances, low power consumption, small airgaps and, in general, compactness. Thus, the controlled DC electromagnet schemes have received more attention than the other techniques of repulsion levitation. Of the many applications investigated three are described here as they illustrate the diverse nature of requirements which may be fulfilled as well as the versatility with which this may be achieved through the use of magnetic suspension.

The first application is that of a flowmeter (Jayawant and Aylwin 1978), in particular for measuring flow rates of the order of $1-3$ mile $\mathrm{h}^{-1}$. A prototype instrument is shown in figure 53 (plate). It consists of a central turbine-type rotor suspended between two controlled DC electromagnets. The electromagnets are pot-core-shaped and have the $I / B$ transducers in the pole face (\$6.3.1.3). The axis of the magnets and the rotor can be horizontal or vertical or at any other angle. Even in the horizontal position there is very little drag due to induced eddy currents and in order to measure the speed of flows some 
controlled eddy current damping must be introduced. Many other geometries are possible and the principal advantages are low power consumption and ruggedness in hostile environments as compared to the alternative of rather high-precision low-friction bearings.

A second application is perhaps at the other end of the speed spectrum and is that of turbomolecular pumps for extremely high vacua. The speed of the rotor is in excess of 10000 RPM and the rotor can not only be held in suspension but also driven by an electric motor such as an induction motor in a completely non-contact manner. If the pump rotor and the driving motor are both in the high-vacuum chamber the problem of seals for conventional bearings can be eliminated. The suspension stiffness required for such an operation is rather high, the airgap clearance is very small and could only be achieved in the restricted space available by using infrared transducers instead of (the conventional) optical ones.

The third application is that of a centrifuge consisting of a steel drum which, when full, weighs over 8 tons. The drum is suspended by two magnets at the top (figure 54 (plate)) and guided by two magnets at each end. The drum is driven by an arch-shaped linear induction motor located in the middle. At its maximum the speed of the $1.6 \mathrm{~m}$ diameter drum is 300 RPM and thus the peripheral speed is approximately 100 mile $^{-1}$. The reasons for adopting magnetic suspension are elimination of noise created when driven by steel rollers on which the drum rests as in the conventional equipment and speeding up the process quite substantially due to the much higher speeds achieved. The airgap between the rails on the drum and the magnets is $7 \mathrm{~mm}$ and the power consumption of each magnet is $900 \mathrm{~W}$ when the drum is stationary.

\section{Assessment of electromagnetic suspension and levitation schemes}

The current level of research activity and popular interest in this field stems primarily from the possibilities in advanced ground transportation schemes as alternatives to the conventional steel-wheel steel-rail form of transport. The activity in electromagnetic methods has increased as it became apparent in the middle to late 1960s that the aircushion systems such as the Tracked Hovercraft in England or the Aerotrain in France have serious limitations and operational difficulties, including noise. Whilst the magnetic river scheme has probably produced more innovative thinking, as shown at the end of $\$ 5.3$, the reactive power input needs to be much reduced before the scheme could be considered as practicable. There is also no known large-scale model of it in operation anywhere to base reasonable estimates of its performance at full scale. Permanent magnet repulsion schemes may have applications in factory floor handling, say trolleys, but again for high-speed or even urban applications it does not seem a likely candidate as yet due to the unsolved problems of track laying and maintenance. This basically leaves only the superconducting magnet levitation and the controlled DC electromagnet schemes as the contenders for advanced ground transportation (AGT) schemes. Superconducting magnet schemes have been full of unsolved problems, not the least those of cryostats, and work on them has come to a standstill except in Japan. The Japanese National Railway scheme has propulsion units on board the vehicle and, therefore, the problem of power collection at speed has still to be tackled. It is, however, possible to invert the arrangement, i.e. to have a long stator, and this arrangement was tested in Erlangen in Germany and is proposed in the Canadian scheme. Neither for this scheme nor for the controlled DC electromagnet scheme employing linear synchronous motors with air-cored winding on the track are any reliable costs available. Until these are 
available it is difficult to foresee whether such schemes could be implemented even on a trans-European basis. The AGT high-speed systems must be considered as being in direct competition with aircraft and it is conceivable that when using aeroplanes to carry passengers is no longer possible due to shortage of fossil fuels, electrically propelled passenger carrying vehicles will be the only possible alternative. In the lower speed range the DC electromagnet scheme is the only possible technology. Here the problems of power pickup do not exist and the track costs also appear to be reduced significantly since it is now the passive element. It may also be possible to combine the lift and propulsion functions in the same magnets. Environmentally, such schemes will be acceptable due to absence of noise and pollution but whether they could be made to fit into existing urban environments remains debatable. Operationally the controlled DC electromagnetic suspension systems offer substantial advantages over any wheeled systems on the grounds of operating costs if fully automated and in terms of reliability and maintenance.

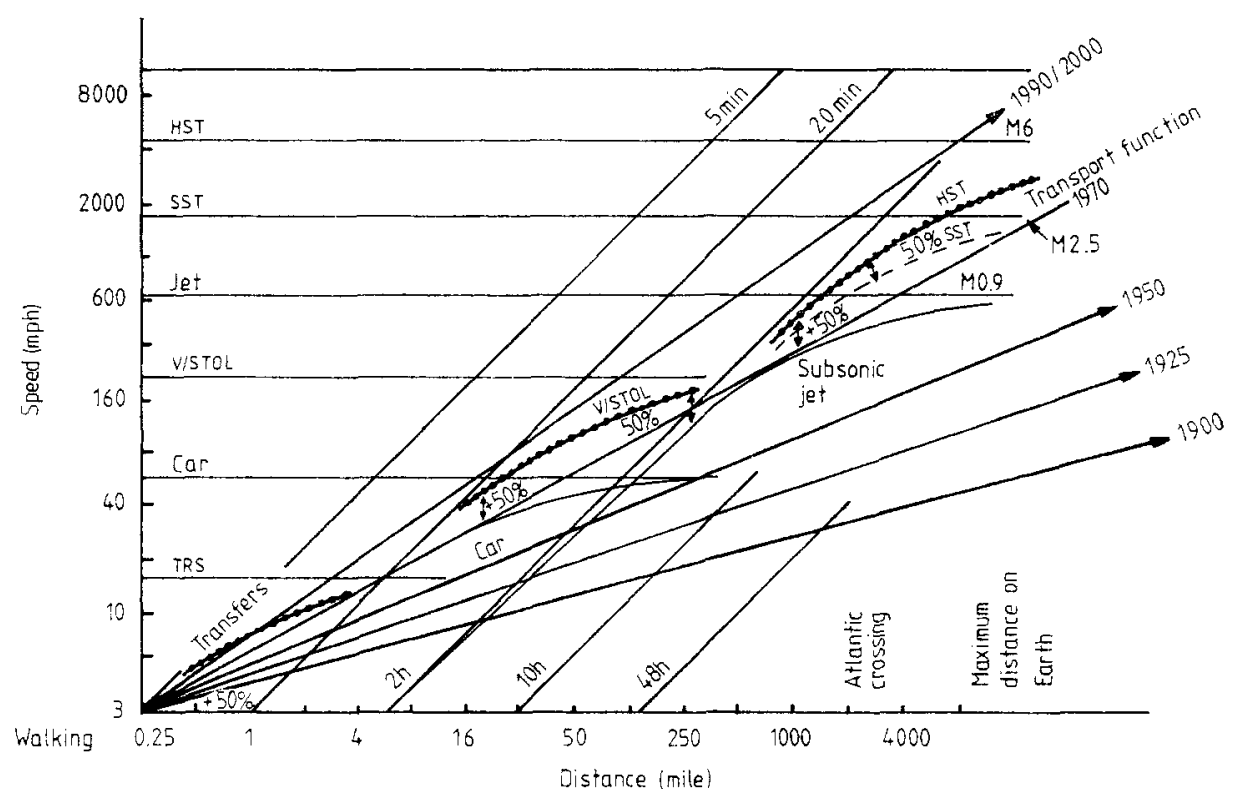

Figure 55. Transport hierarchy.

It is of some interest in passing to take a look at the transport hierarchy of today (figure 55). If it is assumed that people principally use three modes of transportwalking for less than $\frac{1}{4}$ mile, cars for $2 \frac{1}{2}-110$ miles, and aeroplanes for distances of greater than 200 miles-it is seen that there are some significant gaps for both urban and longdistance travel which at present are filled by various means from bicycles to trains and could equally well be filled by electromagnetic systems of the type discussed. An innovatory public transport system must have sufficient passenger attraction potential mainly to attract the motorist from his car for it to be acceptable to transport planners even technically. For this purpose several criteria have been put forward (Grant 1973) which include alignment flexibility to blend new systems into an existing city fabric, visual intrusion, i.e. a light track of minimum cross section, a high frequency of service independent of labour costs which in turn requires ultra-high reliability, small cheap vehicles, a short distance grid, low noise and pollution, ease of switching and maintenance and 
freedom from service interruption due to bad weather. It is interesting to note that no existing system will fulfil all these criteria.

In the application of magnetic suspension systems to frictionless bearings or supports there is a very wide range of applications possible, with controlled DC electromagnet schemes being most predominant. The cost of the suspension element seems to dominate the thinking and for this reason the superconducting magnet schemes do not figure predominantly. Although the control aspect is a good deal more difficult, controlled permanent magnets offer the possibility of very small suspension power requirements. It has already been proved that controlled DC electromagnet schemes are not only low cost but also capable of operating satisfactorily in the most hostile environmental conditions from high vacuum to heavily contaminated atmospheres, from indoors to outdoors and from extremely low temperatures to fairly high ones. The room for further adaptation and innovation remains almost unlimited.

\section{References}

Anschütz-Kaempfe H 1923a British Patent No 193397

- 1923b US Patent No 1589039

Arkadiev V 1945 J. Phys., Moscow 9148 1947 Nature 160330

Backers FT 1961 Philips Tech. Rev. 22 232-8

Bahmanyar H 1973 Novel transport systems with particular reference to contact free suspension. PhD Thesis London University

Bahmanyar H and Ellison AJ 1974 Proc. IEE Conf. on Linear Electric Machines. Publ. No 120 (Stevenage: IEE) pp 203-7

Bamji F 1974 Proc. IEE Conf. on Linear Electric Machines. Publ. No 120 (Stevenage: IEE) pp 68-76

Baran W 1971 Z. Angew. Phys. 32 216-8

Barwell FT and Laithwaite ER 1967 Proc. Inst. Mech. Engrs. $1813 \mathrm{G}$

Barwick RW, McArthur S and Redfern M 1977 British Railways Board Research and Development Division Tech. Rep. TREDYN 7

Beams JW 1937 J. Appl. Phys. 8 795-806

Becker JJ 1970 Sci. Am. Dec, 92-100

Bedford BD, Peer LHB and Tonks L 1939 General Electric Rev. 42246

Bevir MK 1976 UKAEA Culham Laboratory Preprint No CLM-P 458

Boerdijk AH 1956a Philips Res. Rep. 11 45-56

—. 1956b Philips Tech. Rev. 18 125-7

Borcherts R H, Davis LC, Reitz JR and Wilkie DF 1973 Proc. IEEE 61 569-78

Borcherts R H and Davis LC 1973 J. Appl. Phys. 43 2418-27

Braunbeck W 1939a Z. Phys. 112 753-63

-1939b Z. Phys. 112 764-9 1953 Umschau 53 68-70

Buchold TA 1962 US Patent No 3044309

Cambridge Thermionic Corporation 1963 Electronic Design 11 7, 32-3

1975 US Patent No 1401514

Chirgwin KM 1974 Proc. IEE Conf. on Linear Electric Machines. Publ. No 120 (Stevenage: IEE) pp 236-43

Chrisinger JE, Tilton EL, Parkin WJ, Coffin JB and Covert EE 1963 J. R. Aero. Soc. 67 717-24

Clemens PL and Cortner AH 1963 Bibliography Arnold Engineering Center Tech. Documentary Rep. AEDC-TDR-63-20

Coffey HT, Barber TW and Chilton F 1969 J. Appl. Phys. 402161

Covert EE and Finston M 1973 Prog. Aerospace Sci. (Oxford: Pergamon) ch 2, pp27-107

Crain CD 1965 Design and critical calibration of a magnetic suspension system for wind tunnel models AEDC-TR-65-187

Crämer W 1979 Colloq. on advanced ground transportation schemes. IEE Digest No 1979/27

Culver WH and Davis MH 1957 Rand Corporation, Santa Monica, California. Rep. No R363 
Davis LC 1972 J. Appl. Phys. 43 4256-7

Domande H 1973 Proc. Conf. on Personal Rapid Transit, University of Minnesota (Milwaukee: University of Minnesota) pp77-88

Dukes TA and Zapata RN 1969 IEEE Trans. AES-1 20-8

Earnshaw S 1842 Trans. Camb. Phil. Soc. 7 97-112

Eastham JF 1977 Electronics and Power 23 239-42

1978 The magnetic river. Paper presented to the Town meeting of the Science Research Council, UK, Advanced Ground Transport Panel

Eastham A R (ed) 1975 CIGGT Rep. No TS-5

Eastham AR and Atherton DL 1975 IEEE Trans. MAG-11 2

Eastham JF and Balchin MJ 1974 Proc. IEE Conf. on Linear Electric Machines. Publ. No 120 (Stevenage: IEE) pp9-14

Eastham JF and Laithwaite ER 1973 Proc. IEE $120337-43$

- 1974 Proc. IEE 121 1099-108

Eastham AR and Rhodes RG 1971 Proc. 2nd Int. Symp. on electromagnetic suspension, Southampton University (Southampton: Southampton University) ppE1-20

Edwards JD and Antably A el 1978 Proc. IEE 125 209-14

Ellison AJ and Bahmanyar H 1974 Proc. IEE 121 1224-48

Evershed S 1900 J. IEE 29 743-9

Faus HT 1943 US Patent No 2315408

de Ferranti SZ 1947 British Patent No 590292

Forgacs R L 1973 Proc. IEEE 61 604-16

Frazier R H, Gillinson PJ and Oberbeck GA 1974 Magnetic and electric suspension (Cambridge, Mass.: MIT Press)

Freeman EM and Laithwaite ER 1968 Proc. IEE 115538

Freeman EM and Lowther DA 1973 Proc. IEE 120 1499-506

Geary PJ 1964 Survey of Instrument Parts No 6. SIRA Res. Rep. No R314

General Electric Co. (USA) 1963 GE Advanced Technology Laboratory, Schenectady, NY. Tech. Documentation Rep. No ASD-TDR-63

Gillinson PJ, Denhard W G and Frazier RH 1960 MIT Instrument Lab. Rep. No R-272

Gondhalekar VM 1980 Control aspects of magnetically suspended vehicles using controlled d.c. electromagnets. DPhil dissertation University of Sussex

Gottzein E, Brock K H, Schneider E and Pfefferl J 1977 Automatica 13 201-23

Gottzein E and Crämer W 1977 Proc. IFAC Symp. on Multivariable Technological Systems, New Brunswick (Oxford: Pergamon)

Gottzein E, Crämer W, Ossenberg FW and Roche C 1975 Proc. IUTAM Symp. on the dynamics of vehicles on roads and railway tracks. Delft (Amsterdam: Swets and Zeitlinger) pp504-30

Gottzein E and Lange B 1975 Automatica 11 271-84

Gottzein E, Meisinger R and Miller L 1979 ZEV-Glasers Ann. 103 227-32

Graeminger B 1912 British Patent No 74499, 24541

Grant BE 1973 Proc. Symp. on Advanced Transport Systems, Warwick University (Coventry: Warwick University) pp22-38

Greene A H 1974 IEEE Trans. MAG-10 431-4

Guderjahn CA, Wipf S L, Fink HJ, Boom RW, McKenzie KE, Williams D and Downey T $1969 \mathrm{~J}$. Appl. Phys. $402133-40$

Guthberlet HG 1974 IEEE Trans. MAG-10 417-20

Hagihara S 1974 Elec. Engng, Japan 95 493-9

Harding JT and Tuffias RH 1960 California Institute of Technology, Jet Propulsion Laboratory, Tech. Release No 34-100

Hazlerigg ADG 1974 Proc. IEE Conf. on Control Aspects of Guided Land Transport. Publ. No 117 (Stevenage: IEE) pp233-9

Hodkinson RL 1972 Suspension and stabilisation of an electric train by electromagnets. MSc dissertation University of Sussex

- 1974 Proc. IEE Conf. on Control Aspects of Guided Land Transport. Publ. No 117 (Stevenage: IEE) pp184-92

Holmes FT 1937 Rev. Sci. Instrum. 8 4441-7

Homer JG, Rendle TC, Walters CR, Wilson MN and Bevir MK 1977 J. Phys. D: Appl. Phys. 10 879-86

Jayawant BV 1977 Electronics and Power 23 236-8 


\section{British Patent No 1557864}

1981 Electromagnetic suspension and levitation (London: Edward Arnold)

Jayawant BV and Aylwin DG 1978 British Patent No 1497801

Jayawant BV and Rea D P 1968 Proc. IEE 115 549-54

Jayawant BV and Sinha PK 1977 Automatica 13 605-10

Jayawant BV, Sinha PK and Aylwin D G 1976 Int. J. Control 24 627-39

Jayawant BV, Sinha PK, Wheeler AR, Whorlow RJ and Willsher J 1975 Proc. IEE 123 941-8

Jenkins AW and Parker HM 1969 J. Appl. Phys. Suppl. 302385

Judd M and Goodyear M 1965 Magnetic suspension system. Annual Report of the Aeronautics Department, Southampton University

Kammerlingh Onnes H 1911 Konik. Akad. Wetensch. Amsterdam Proc. 14 Comm. No 122b 113-5

Kaplan BZ 1967 Proc. IEE $1141801-4$

1970 Electron. Lett. 670

Katz R M, Nene VD, Ravera RJ and Skalski CA 1974 Trans. ASME 96 G2, 204-12

Kellogg OD 1953 Foundations of potential theory (New York: Dover)

Kemper H 1937 German Patent No 643316, 644302

1938 Schwebende auf hängung durch electro-magnetische kräft; eine möglichkeit für eine grundsätlich neue fort bewegungsart. ETZ 59, 15, 391-5

Kilgore RA and Hamlet JL 1966 Summary of ARL Symp. on magnetic wind tunnel model suspension and balance systems ed F L Daum (Cambridge, Mass.: MIT) ARL 66-0135

Knobel HW 1964 Control Engng 11 70-1

Kunzler JE 1961 Rev. Mod. Phys. 33 501-9

Laithwaite ER 1965 Proc. IEE 112 2361-75

1966 Induction machines for special purposes (Newnes)

- 1973a High speed ground transport. Royal Institution discourse

1973b Electronics and Power $19310-2$

- 1977 Transport without wheels (Elek Science) ch 11

Laithwaite ER, Eastham JF, Bolton H and Fellows T G 1971 Proc. IEE 118 1761-7

Linder D 1976 Proc. 2nd IEE Conf. on Advances in Magnetic Materials and their Applications. Publ. No 142 (Stevenage: IEE) pp96-9

London F 1961 Superfluids (New York: Dover)

Mathias BT 1957 Sci. Am. 197 May, 92-103

McCaig M 1961 Elec. Rev. 169425

1967 Permanent magnets for repulsion devices. Swift and Levick Bulletin

Meisinger R 1975 Proc. IUTAM Symp. on the dynamics of vehicles on roads and railway tracks, Delft (Amsterdam: Swets and Zeitlinger) pp531-54

1977 Beiträge zur reglung einer magnet schwebebahn auf elastischem fahrweg. Dr. Ing. Dissertation Technische Universität München

Meissner W and Ochsenfeld R 1933 Naturw. 2178

Nakamura S 1979 IEEE Trans. MAG-15 1428-33

Nakamura S, Takeuchi Y and Takahashi M 1979 IEEE Trans. MAG-15 1434-6

Nordsiek AT 1961 American Rocket Society, Guidance, Control and Navigation Conf., Stanford, Calif. (New York: ARS) Paper No 1963-61

Ohno E, Iwomoto M and Yamada T 1973 Proc. IEEE 61 579-86

ONERA 1960 Engineer 210 5463, 67-8

Ooi BT and Banakar MH 1975 IEEE Trans. MAG-11 1418-20

Orkress EC, Wroughton D M, Coment ZG, Brace PH and Kelly JCR 1952 J. Appl. Phys. 231413

Outsuka T and Kyotani Y 1975 IEEE Trans. MAG-11 608-14

Papas C H 1977 Appl. Phys. 13 361-4

Pfann WG and Hagelbarger DW 1956 J. Appl. Phys. 27 12-8

Polgreen GR 1965 Transport possibilities with magnetic suspension. Electrical Times 25 August

- 1966a New applications of modern magnets (London: McDonald)

- 1966b Proc. Inst. Mech. Engrs 181 3G, 145-50

1968 Engineer 226 632-6

1971 Electronics and Power 127 233-7

Polonis DH, Butters RG and Parr JG 1954 Research 7272

Popp K and Schiehlen W 1975 Proc. IUTAM Symp. on the dynamics of vehicles on roads and railway tracks, Delft (Amsterdam: Swets and Zeitlinger) pp479-503

Powell JR 1963 The magnetic road. IEEE Paper 63-RR-4 
Powell JR and Danby GR 1966 ASME Publ. 66WA/RR5

Reitz JR 1970 J. Appl. Phys. 41 2067-71

Reitz JR and Borcherts R H 1975 IEEE Trans. MAG-11 615-8

Reitz JR, Borcherts RH, Davis LC and Wilkie DF 1972 Ford Motor Co., Tech. Rep. No FRA-RT-72-40

Reitz J R and Davis LC 1972 J. Appl. Phys. 43 1547-53

Rhodes R G 1976 Proc, 6th Int. Cryogenic Engineering Conf., Grenoble (Grenoble: University of Grenoble) pp63-7

Rhodes R G, Mulhall B and Abel E 1974 Proc. IEE Conf. on Control Aspects of Guided Land Transport. Publ. No 117 (Stevenage: IEE) pp214-20

Ross JA 1973 Proc. IEEE 61 617-20

Rutherford Laboratory and Culham Laboratory 1976 A new system for magnetic levitation. Brochure for the Royal Society Conversazione

Schoenberg D 1960 Superconductivity (Cambridge: Cambridge University Press) pp16-20

Schreibe W 1953 Metall. 7751

Silver KR and Henderson JG 1969 J. Aircraft 6398

Simon I 1953 J. Appl. Phys. 24 19-24

Sinha PK 1977a Proc. 4th MVTS Symposium (IFAC), New Brunswick (Oxford: Pergamon) pp573-81

- 1977b Proc. IEE Conf. on New Developments in Automatic Testing. Publ. No 158 (Stevenage: IEE) pp22-3

Skinner D J and Edwards D R 1978 Discussion at a meeting on Superconducting Cables, IEE Savoy Place, London

Slemon GR 1975 Trans. IEEE MAG-11 1478-83

Tang $\mathrm{CH}$, Harold W J and Chu RS 1975 Trans. IEEE MAG-11 625-6

Temple G and Bickley W 1933 Rayleigh's principle (Oxford: Oxford University Press)

Thornton R D 1973 Proc. IEEE 61 586-98

Tournier M and Laurenceau P 1957 Recherche Aeronautique 59 21-6

University of Virginia 1962 Tech. documentary rep. for Aeronautical Systems Division, Air Force Systems Command, Wright Patterson Base, Ohio, No ASD-TDR-62-441

Uranker L 1974 IEEE Trans. MAG-10 421-4

Voigt H 1974 Wiss-ber-AEG-Telefunken 47 15-20

Weisberg L R 1959 Rev. Sci. Instrum. 30135

West JC and Hesmondhalgh DE 1962 Proc. IEE 109C 172-81

West JC and Jayawant BV 1962 Proc. IEE 109A 292-300

Westinghouse Engineer 1965 Westinghouse Engineer 25 95-6

Whorlow RJ 1978 Design of magnetic suspension systems and control of vehicles in networks. DPhil Dissertation University of Sussex

Wilson A and Luff B 1966 Royal Aircraft Establishment, Farnborough, Tech. Rep. No. TN66248

Yamamura S 1976 IEEE Trans. MAG-12 874-8

Yamamura S and Ito T 1975 Proc. INTERMAG Conf. (New York: IEEE) Paper No 28. 1 


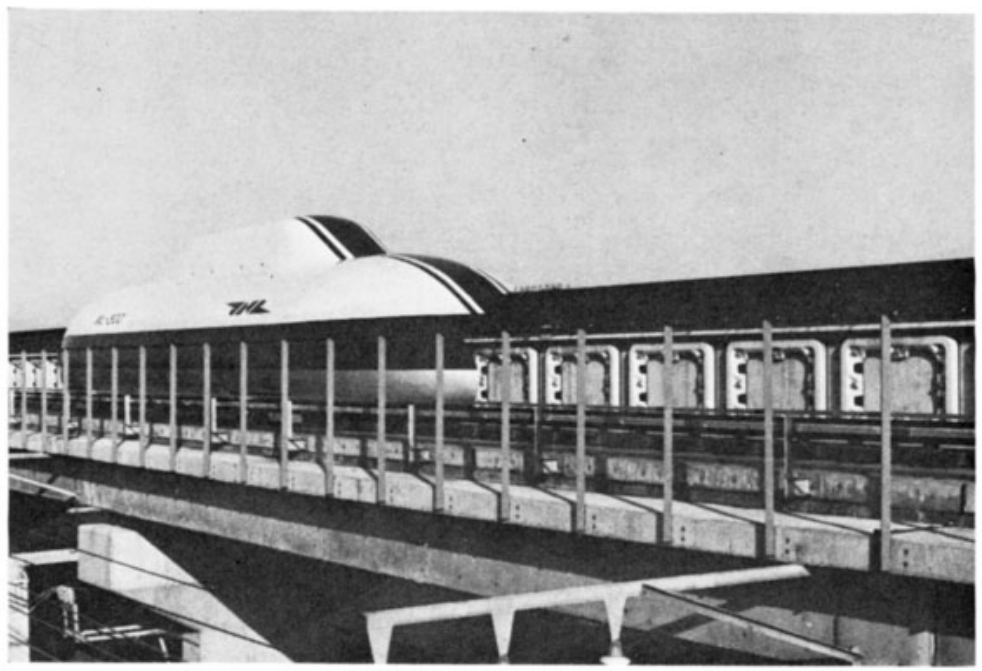

Figure 3. Japanese National Railways superconducting magnet vehicle. (Reproduced by permission of the Japanese National Railways.)

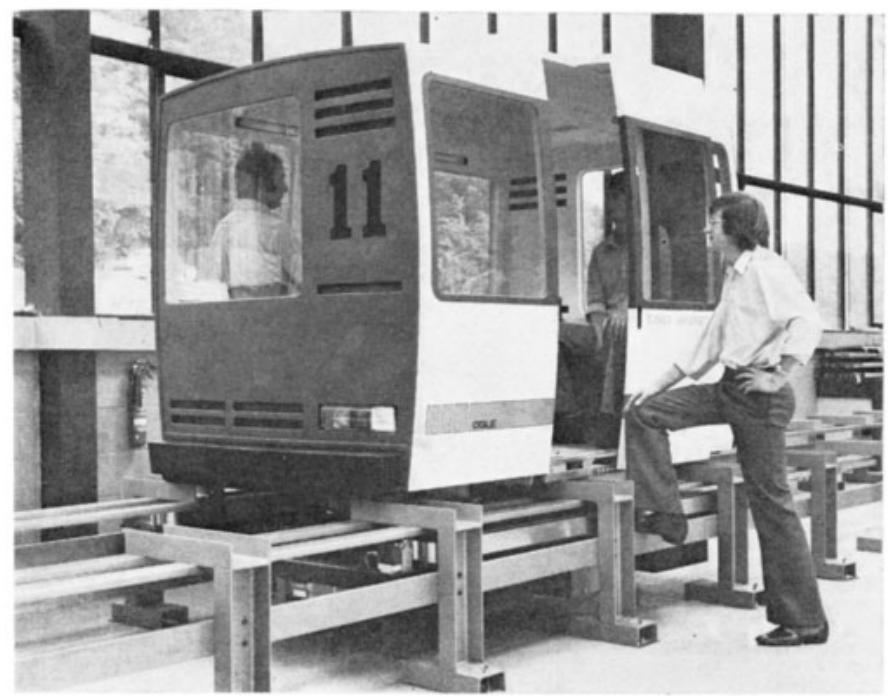

Figure 6. University of Sussex 1 ton 4 passenger vehicle using controlled Dc electromagnets for suspension. 


\section{B V Jayawant}

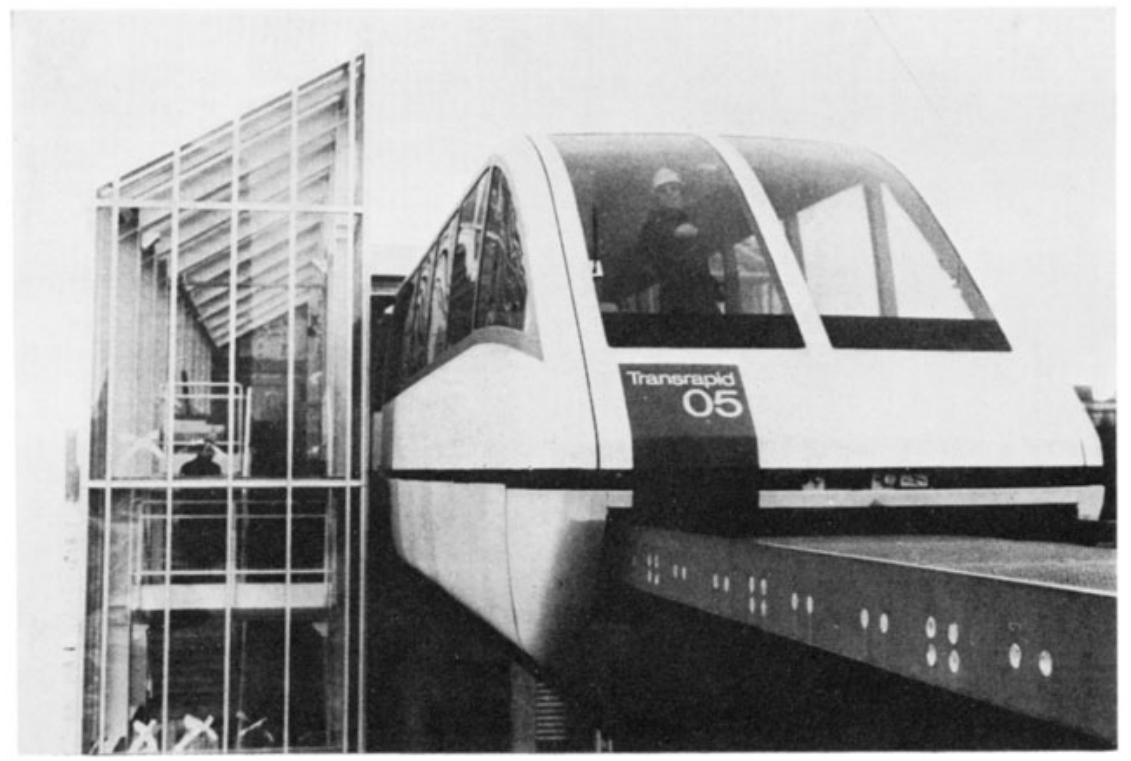

Figure 7. MBB, 35 ton 68 passenger vehicle demonstrated in Hamburg Exhibition in 1979. (Reproduced by permission of MBB.)

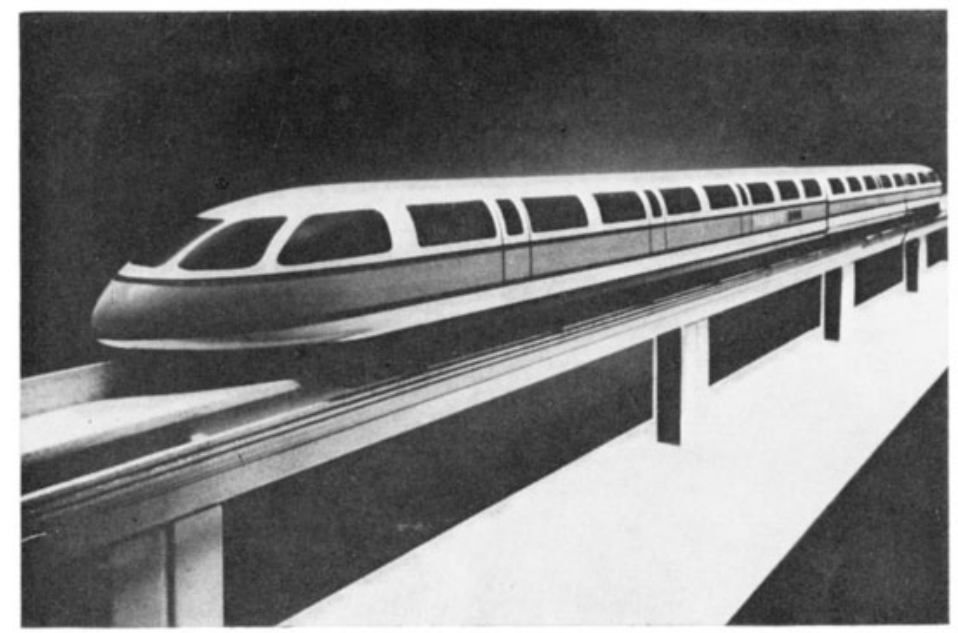

Figure 8. Model of the German 121 ton vehicle for the Emsland project. (Reproduced by permission of MBB.) 


\section{Electromagnetic suspension and levitation}

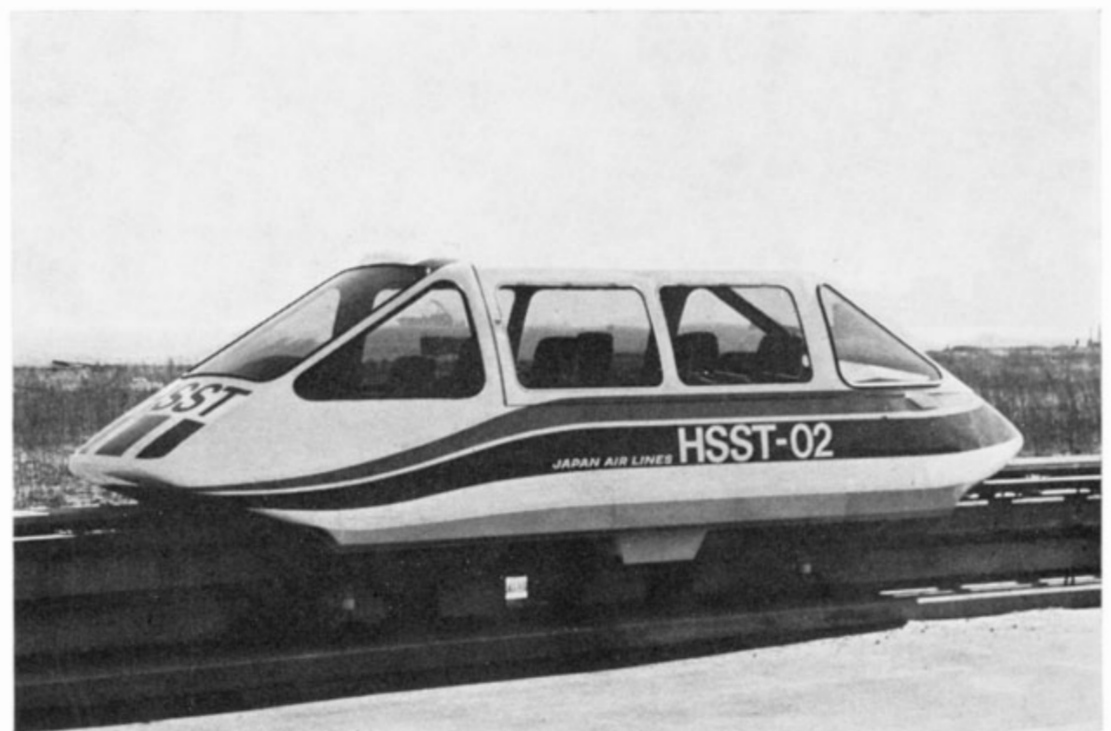

Figure 9. Japan Air Lines 8 passenger vehicle HSST-02. (Reproduced by permission of Japan Air Lines.) 


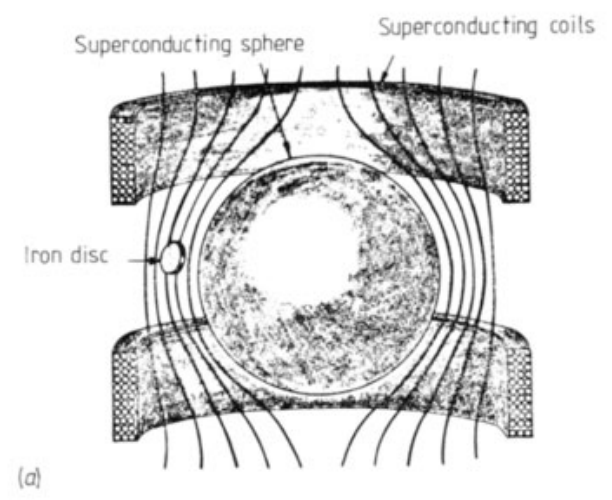

(b)
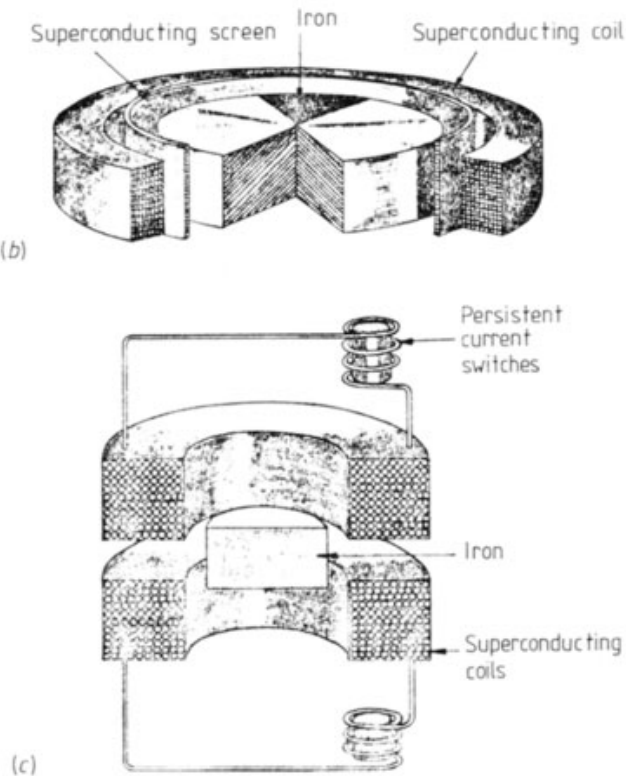

Figure 11. Mixed $\mu$ levitation. (a) Iron disc suspended near a superconducting sphere. (b) iron disc suspended inside a (superconducting) magnetic flux screen, $(c)$ iron hody suspended between two constant flux coils. 


\section{Electromagnetic suspension and levitation}

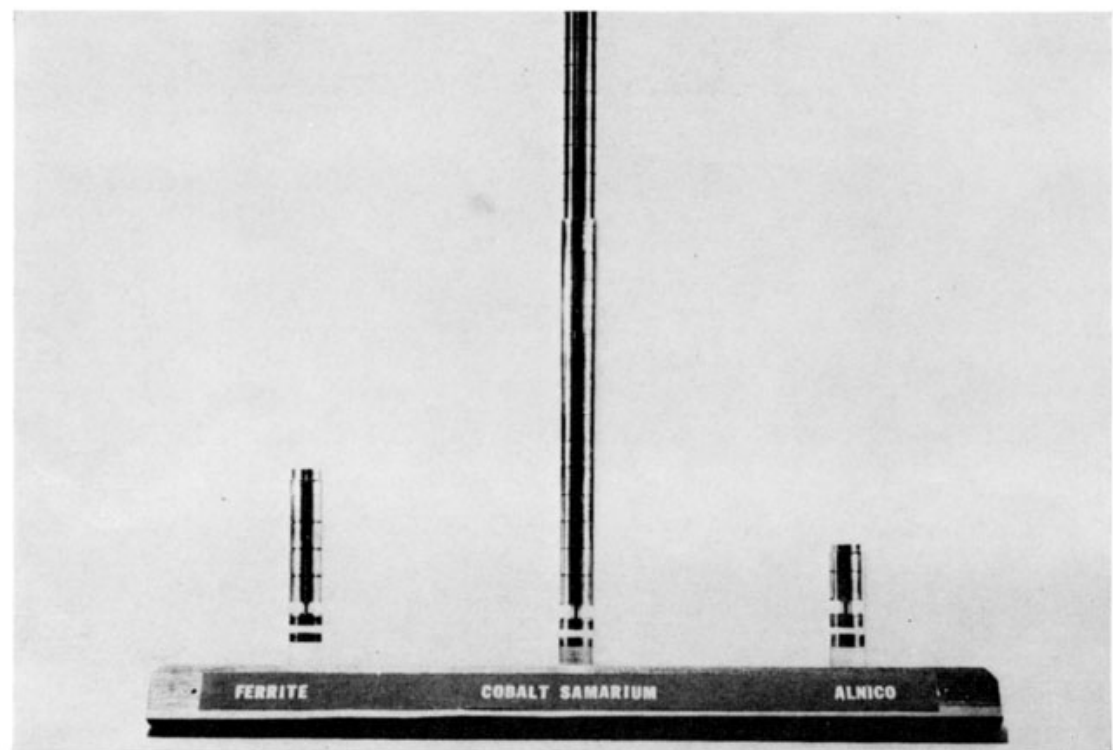

Figure 13. Relative weights which can be supported by permanent magnets made of Alnico, barium ferrite and cobalt samarium in repulsion. (Reproduced by permission of $\mathbf{J}$ Becker.) 


\section{B V Jayawant}

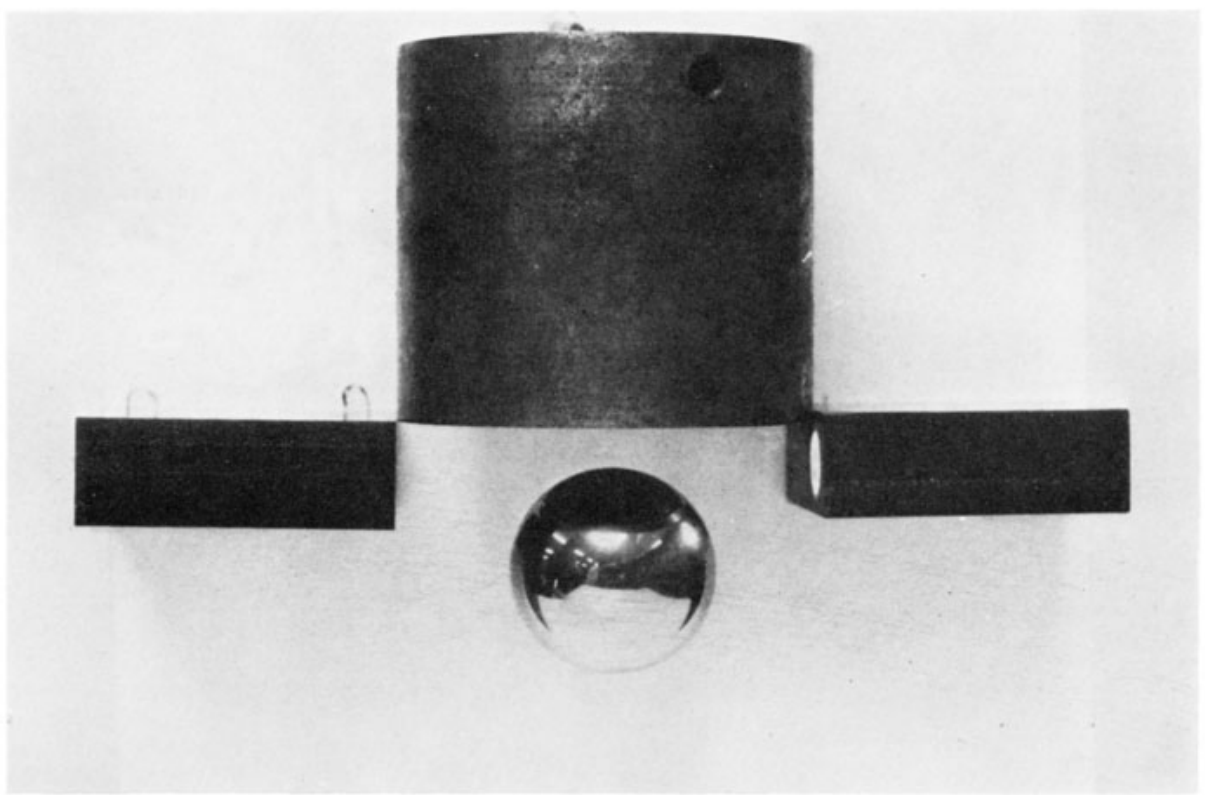

Figure 40. Steel ball suspended under a controlled DC electromagnet.

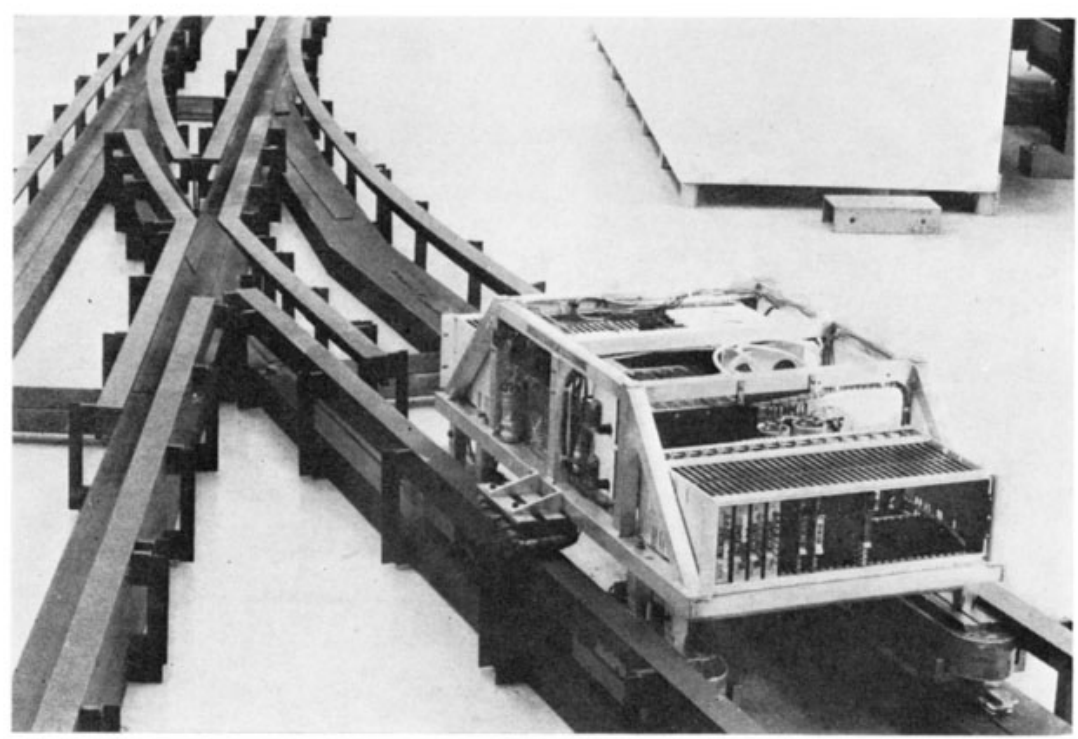

Figure 51. I-magnet vehicle with a junction in the track. 


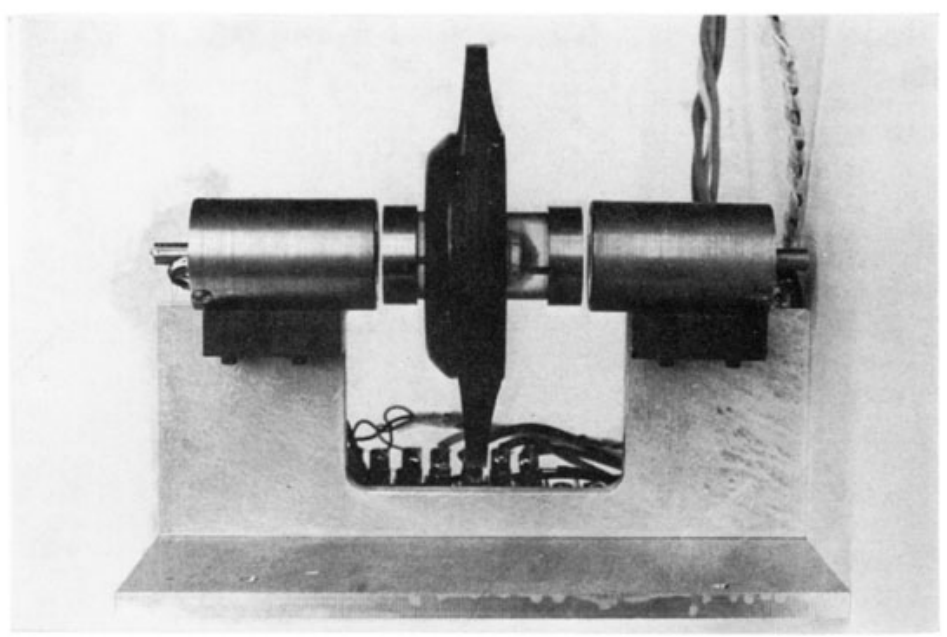

Figure 53. A turbine rotor flowmeter.

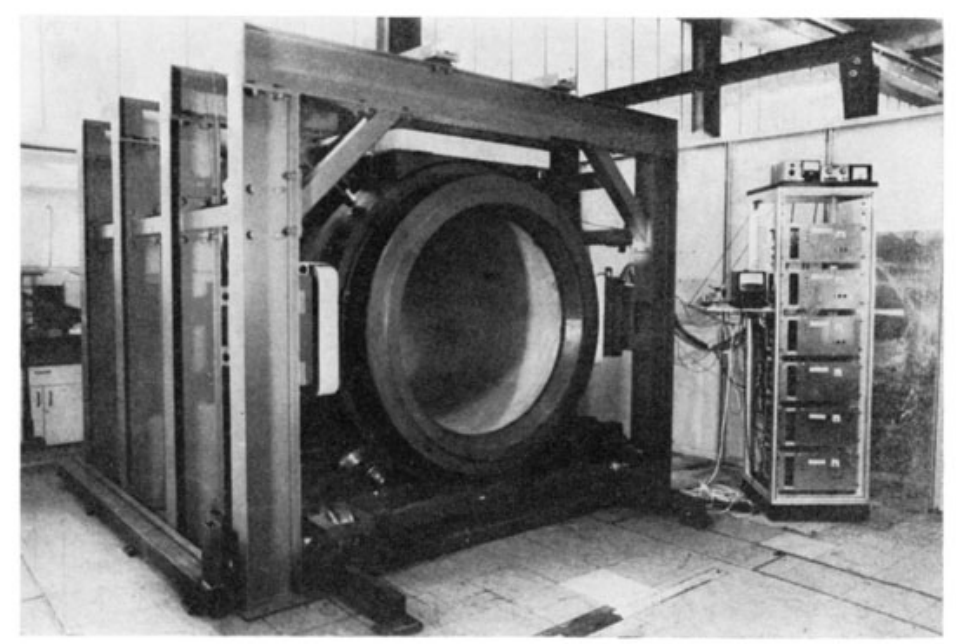

Figure 54. 8 ton suspended steel drum centrifuge. 